\title{
Accidental matter at the LHC
}

\author{
Luca Di Luzio, ${ }^{a}$ Ramona Gröber, ${ }^{b}$ Jernej F. Kamenik ${ }^{c, d, e}$ and Marco Nardecchia ${ }^{f}$ \\ ${ }^{a}$ Dipartimento di Fisica, Università di Genova and INFN — Sezione di Genova, \\ via Dodecaneso 33, 16159 Genova, Italy \\ ${ }^{b}$ INFN - Sezione di Roma Tre, \\ via della Vasca Navale 84,00146 Roma, Italy \\ ${ }^{c}$ Jožef Stefan Institute, \\ Jamova 39, 1000 Ljubljana, Slovenia \\ ${ }^{d}$ Faculty of Mathematics and Physics, University of Ljubljana, \\ Jadranska 19, 1000 Ljubljana, Slovenia \\ ${ }^{e}$ CERN TH-PH Division, \\ Meyrin, Switzerland \\ ${ }^{f}$ DAMTP, University of Cambridge, \\ Wilberforce Road, Cambridge CB3 OWA, U.K. \\ E-mail: luca.di.luzio@ge.infn.it, ramona.groeber@roma3.infn.it, \\ jernej.kamenik@cern.ch, M.nardecchia@damtp.cam.ac.uk
}

ABSTRACT: We classify weak-scale extensions of the Standard Model which automatically preserve its accidental and approximate symmetry structure at the renormalizable level and which are hence invisible to low-energy indirect probes. By requiring the consistency of the effective field theory up to scales of $\Lambda_{\text {eff }} \approx 10^{15} \mathrm{GeV}$ and after applying cosmological constraints, we arrive at a finite set of possibilities that we analyze in detail. One of the most striking signatures of this framework is the presence of new charged and/or colored states which can be efficiently produced in high-energy particle colliders and which are stable on the scale of detectors.

KEYwords: Beyond Standard Model, Effective field theories

ARXiv EPrint: 1504.00359 


\section{Contents}

1 Introduction 1

2 Accidentally safe extensions of the SM 5

2.1 New fermions 6

2.2 New scalars 8

$\begin{array}{llr}\text { 2.2.1 Scalar potential, CP and custodial symmetry } & 8\end{array}$

$\begin{array}{ll}2.2 .2 \text { Bounds on Higgs portal coupling } & 11\end{array}$

$\begin{array}{lll}2.3 \text { Mass spectrum } & 13\end{array}$

2.4 Validity of the EFT 14

3 Lifetimes $\quad 16$

$\begin{array}{lll}3.1 & \text { Inter-multiplet weak transitions } & 16\end{array}$

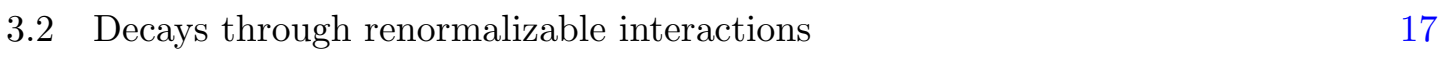

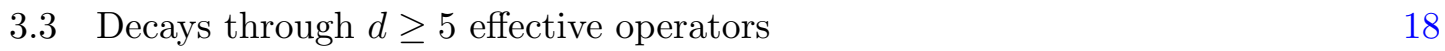

$\begin{array}{lll}3.3 .1 & \text { Cascade decays } & 19\end{array}$

$\begin{array}{ll}3.3 .2 & \text { Loop-induced decays } \\ & 21\end{array}$

4 Cosmology 23

4.1 Relic abundance 24

$\begin{array}{lll}4.2 & \text { Implications for Big Bang nucleosynthesis } & 27\end{array}$

5 Collider phenomenology $\quad 30$

5.1 Renormalizable cases 31

5.2 Colorless and charged LP 32

5.3 Colorless and neutral LP 33

$\begin{array}{lll}5.4 & \text { Colored LP } & 35\end{array}$

6 Conclusions $\quad 39$

$\begin{array}{ll}\text { A Two-loop Landau poles } & 41\end{array}$

B SU $(2)_{L}$ decompositions $\quad 42$

\section{Introduction}

The electroweak (EW) hierarchy puzzle suggests that new physics (NP) degrees of freedom should appear around or not much above the EW scale. Hence the search for NP is a clear target being vigorously pursued by the LHC experiments. On the other hand, indirect searches for NP using flavor and CP violating observables have already probed NP scales 
up to $10^{8} \mathrm{GeV}$ (cf. [1-3] for recent reviews). Thus, the overall excellent agreement with the CKM paradigm predictions suggests a large mass gap above the EW scale. Perhaps even more strikingly, searches for baryon (B) and lepton (L) number violating processes at low energies suggest that these accidental quantum numbers of the standard model (SM) are good symmetries of nature up to scales of the order of $10^{15} \mathrm{GeV}$ (cf. [2]).

Explicit models of $\mathrm{TeV}$ scale NP need to resolve the apparent conflict between these two sets of expectations by postulating exact or approximate symmetries which in term forbid or sufficiently suppress the most dangerous contributions to flavor changing neutral currents, CP violation, as well as $\mathrm{B}$ and $\mathrm{L}$ changing processes. These include explicit $\mathrm{B}, \mathrm{L}$ or their anomaly free combinations, discrete space-time symmetries including $\mathrm{C}, \mathrm{P}$, CP but also new internal symmetries like R-parity (or R-symmetry) in supersymmetric (SUSY) extensions of the SM, KK-parity in extra-dimensional setups, as well as abelian or non-abelian horizontal (flavor) symmetries.

At the heart of these problems is the fact that generically, extending the SM particle content will either (i) break some of the SM accidental symmetries, and/or (ii) introduce new sources of breaking of the approximate SM symmetries, which in general will not be aligned with existing SM symmetry breaking directions. Examples of the first kind include B and L. Flavor, CP and custodial symmetry of the Higgs potential fall into the second category.

Consider the SM as the renormalizable part of an effective field theory (EFT)

$$
\mathcal{L}=\mathcal{L}_{\mathrm{SM}}^{(d \leq 4)}+\sum_{d>4} \frac{1}{\Lambda_{\mathrm{eff}}^{d-4}} \mathcal{L}^{(d)}
$$

where only SM fields appear as dynamical degrees of freedom in $\mathcal{L}$, and $d$ denotes the canonical operator dimension. Assuming $\mathcal{O}(1)$ coefficients in the EFT operator expansion, currently all experimental evidence in particle physics can be accommodated by such a generic theory with a very large cut-off $\Lambda_{\text {eff }} \approx 10^{15} \mathrm{GeV} .{ }^{1}$ This particular scale is intriguing since it can account for both the observed neutrino masses suggesting the presence of $\mathrm{L}$ violating $\mathcal{L}^{(5)}$, as well as null results of all flavor, $\mathrm{CP}$ and $\mathrm{B}$ violation probes constraining $\mathcal{L}^{(d \geq 6)}$. One may thus ask the following well defined question. Which extensions of the $S M$ particle content with masses close to the EW scale (i) form consistent EFTs with a cut-off scale as high as $10^{15} \mathrm{GeV}$, (ii) automatically preserve the accidental and approximate symmetry structure of the SM and thus do not require the introduction of additional protective mechanisms in order to remain viable LHC targets in light of negative search results of the numerous indirect probes, and (iii) are cosmologically viable?

In the present paper we explore such possibilities by adding to the SM EW (and possibly color) multiplets, requiring that their SM gauge quantum numbers alone forbid all renormalizable interactions which would break any of the SM approximate or accidental symmetries. In particular, in the SM the global $\mathcal{G}_{F} \equiv \mathrm{U}(3)^{5}$ flavor symmetry of quarks and leptons is only broken by their respective Yukawas (and the gauging of hypercharge).

\footnotetext{
${ }^{1}$ Cosmological observations suggest that most of the mass in the observable Universe cannot be accounted for by known forms of matter. The possibility of particle dark matter within our setup is briefly discussed below.
} 


\begin{tabular}{|c|c|c|c|c|c|}
\hline Spin & $\chi$ & $Q_{\mathrm{LP}}$ & $\mathcal{O}_{\text {decay }}$ & $\operatorname{dim}\left(\mathcal{O}_{\text {decay }}\right)$ & $\Lambda_{\text {Landau }}^{2-\text { loop }}[\mathrm{GeV}]$ \\
\hline 0 & $(1,1,0)$ & 0 & $\chi H H^{\dagger}$ & 3 & $\gg m_{\mathrm{Pl}}\left(g_{1}\right)$ \\
0 & $(1,3,0)^{\ddagger}$ & 0,1 & $\chi H H^{\dagger}$ & 3 & $\gg m_{\mathrm{Pl}}\left(g_{1}\right)$ \\
0 & $(1,4,1 / 2)^{\ddagger}$ & $-1,0,1,2$ & $\chi H H^{\dagger} H^{\dagger}$ & 4 & $\gg m_{\mathrm{Pl}}\left(g_{1}\right)$ \\
0 & $(1,4,3 / 2)^{\ddagger}$ & $0,1,2,3$ & $\chi H^{\dagger} H^{\dagger} H^{\dagger}$ & 4 & $\gg m_{\mathrm{Pl}}\left(g_{1}\right)$ \\
\hline 0 & $(1,2,3 / 2)$ & 1,2 & $\chi H^{\dagger} \ell \ell, \chi^{\dagger} H^{\dagger} e^{c} e^{c}, D^{\mu} \chi^{\dagger} \ell^{\dagger} \bar{\sigma}_{\mu} e^{c}$ & 5 & $\gg m_{\mathrm{Pl}}\left(g_{1}\right)$ \\
0 & $(1,2,5 / 2)$ & 2,3 & $\chi^{\dagger} H e^{c} e^{c}$ & 5 & $\gg m_{\mathrm{Pl}}\left(g_{1}\right)$ \\
0 & $(1,5,0)$ & $0,1,2$ & $\chi H H H^{\dagger} H^{\dagger}, \chi W^{\mu \nu} W_{\mu \nu}, \chi^{3} H^{\dagger} H$ & 5 & $\gg m_{\mathrm{Pl}}\left(g_{1}\right)$ \\
0 & $(1,5,1)$ & $-1,0,1,2,3$ & $\chi^{\dagger} H H H H^{\dagger}, \chi \chi \chi^{\dagger} H^{\dagger} H^{\dagger}$ & 5 & $\gg m_{\mathrm{Pl}}\left(g_{1}\right)$ \\
0 & $(1,5,2)$ & $0,1,2,3,4$ & $\chi^{\dagger} H H H H$ & 5 & $3.5 \times 10^{18}\left(g_{1}\right)$ \\
0 & $(1,7,0)^{\star}$ & $0,1,2,3$ & $\chi^{3} H^{\dagger} H$ & 5 & $1.4 \times 10^{16}\left(g_{2}\right)$ \\
\hline $1 / 2$ & $(1,4,1 / 2)$ & -1 & $\chi^{c} \ell H H, \chi \ell H^{\dagger} H, \chi \sigma^{\mu \nu} \ell W_{\mu \nu}$ & 5 & $8.1 \times 10^{18}\left(g_{2}\right)$ \\
$1 / 2$ & $(1,4,3 / 2)$ & 0 & $\chi \ell H^{\dagger} H^{\dagger}$ & 5 & $2.7 \times 10^{15}\left(g_{1}\right)$ \\
$1 / 2$ & $(1,5,0)$ & 0 & $\chi \ell H H H^{\dagger}, \chi \sigma^{\mu \nu} \ell H W_{\mu \nu}$ & 6 & $8.3 \times 10^{17}\left(g_{2}\right)$ \\
\hline
\end{tabular}

Table 1. List of new weak-scale uncolored states $\chi$ which can couple to SM fields at the renormalizable level without breaking $\mathcal{G}_{\mathcal{F}}$, and which are compatible with cosmology and an EFT cut-off scale of $\Lambda_{\text {eff }} \simeq 10^{15} \mathrm{GeV}$. The possible electromagnetic charges of the LP in the multiplet are denoted by $Q_{\mathrm{LP}}$, while $\mathcal{O}_{\text {decay }}$ denotes the lowest dimensional operators responsible for the decay of $\chi$. States with $Y=0$ are understood to be real. In the last column, the Landau pole has been estimated at two loops by integrating in the new multiplet at the scale of the $Z$ boson mass $m_{Z}$, while the symbol in the bracket stands for the gauge coupling, $g_{1,2,3}$, triggering the Landau pole and $m_{\mathrm{Pl}}=1.22 \times 10^{19} \mathrm{GeV}$ is the Planck mass. The states marked with $\ddagger$ and $\star$ are constrained by EW precision tests and BBN, respectively, to lie possibly beyond the LHC reach.

In order not to introduce new sources of $\mathcal{G}_{F}$ breaking, one should only consider $\mathcal{G}_{F}$ singlet operator extensions of the SM. In section 2 we list all $d \leq 3$ operators involving quark and lepton fields that transform nontrivially under $\mathcal{G}_{F}$ and demand that the new degrees of freedom do not couple to any of these at the renormalizable level. Since both $\mathrm{B}$ and $\mathrm{L}$ are subgroups of $\mathcal{G}_{F}$ the above prescription also automatically preserves these accidental symmetries of the SM. Furthermore, in most cases this also ensures the absence of new sources of breaking for both custodial and CP symmetries at the renormalizable level. The exceptions where new breaking can arise in the scalar potential are discussed in section 2.2.1.

The relevant Lagrangian is restricted only by imposing the SM gauge and Lorentz invariance, not by new symmetries. Finally, such a theory is assumed to represent a consistent description of nature up to the cut-off scale $\Lambda_{\text {eff }}$. In particular, we require that all of the marginal couplings (in particular the SM gauge couplings) remain perturbative up to $\Lambda_{\text {eff }}$. The physical idea behind this requirement is that a Landau pole might be associated with the emergence of some new, generic dynamics that will break the accidental symmetries of the SM at scales above the Landau pole. As we show in section 2.4, this condition (together with the cosmological constraints on stable charged particles) limits the size of the new representations and leads to a finite list of possible SM extensions.

Interestingly, it turns out that such SM extensions generically possess extended accidental symmetries which ensure the stability of the lightest particles (LPs) in the new 


\begin{tabular}{|c|c|c|c|c|c|}
\hline Spin & $\chi$ & $Q_{\mathrm{LP}}$ & $\mathcal{O}_{\text {decay }}$ & $\operatorname{dim}\left(\mathcal{O}_{\text {decay }}\right)$ & $\Lambda_{\text {Landau }}^{2-\text { loop }}[\mathrm{GeV}]$ \\
\hline 0 & $(3,1,5 / 3)$ & $5 / 3$ & $\begin{array}{c}\chi^{\dagger} H q e^{c}, \chi H^{\dagger} u^{c} \ell, \\
D^{\mu} \chi^{\dagger} u^{c \dagger} \bar{\sigma}_{\mu} e^{c}\end{array}$ & 5 & $\gg m_{\mathrm{Pl}}\left(g_{1}\right)$ \\
\hline 0 & $(\overline{3}, 2,5 / 6)$ & $1 / 3,4 / 3$ & $\begin{array}{c}\chi^{\dagger} H q q, \chi^{\dagger} H u^{c} e^{c}, \chi H^{\dagger} q \ell, \\
\chi H^{\dagger} u^{c} d^{c}, \chi H u^{c} u^{c}, \\
\chi^{\dagger} H^{\dagger} d^{c} e^{c}, D^{\mu} \chi q^{\dagger} \bar{\sigma}_{\mu} u^{c}, \\
D^{\mu} \chi^{\dagger} q^{\dagger} \bar{\sigma}_{\mu} e^{c}, D^{\mu} \chi d^{c \dagger} \bar{\sigma}_{\mu} \ell\end{array}$ & 5 & $\gg m_{\mathrm{Pl}}\left(g_{1}\right)$ \\
\hline 0 & $(\overline{3}, 2,11 / 6)$ & $4 / 3,7 / 3$ & $\chi H^{\dagger} u^{c} u^{c}, \chi^{\dagger} H d^{c} e^{c}$ & 5 & $5.5 \times 10^{19}\left(g_{1}\right)$ \\
\hline 0 & $(3,3,2 / 3)$ & $-1 / 3,2 / 3,5 / 3$ & $\begin{array}{c}\chi^{\dagger} H^{\dagger} q e^{c}, \chi H u^{c} \ell, \\
\chi H^{\dagger} d^{c} \ell, D^{\mu} \chi q^{\dagger} \bar{\sigma}_{\mu} \ell\end{array}$ & 5 & $\gg m_{\mathrm{Pl}}\left(g_{1}\right)$ \\
\hline 0 & $(3,3,5 / 3)$ & $2 / 3,5 / 3,8 / 3$ & $\chi^{\dagger} H q e^{c}, \chi H^{\dagger} u^{c} \ell$ & 5 & $3.2 \times 10^{17}\left(g_{1}\right)$ \\
\hline 0 & $(3,4,1 / 6)$ & $\begin{array}{c}-4 / 3,-1 / 3 \\
2 / 3,5 / 3\end{array}$ & $\chi H^{\dagger} q q, \chi^{\dagger} H q \ell$ & 5 & $\gg m_{\mathrm{Pl}}\left(g_{2}\right)$ \\
\hline 0 & $(\overline{3}, 4,5 / 6)$ & $\begin{array}{c}-2 / 3,1 / 3 \\
4 / 3,7 / 3\end{array}$ & $\chi^{\dagger} H q q, \chi H^{\dagger} q \ell$ & 5 & $\gg m_{\mathrm{Pl}}\left(g_{2}\right)$ \\
\hline 0 & $(\overline{6}, 2,1 / 6)$ & $-1 / 3,2 / 3$ & $\begin{array}{cl}\chi H^{\dagger} q q, & \chi^{\dagger} H u^{c} d^{c}, \\
\chi^{\dagger} H^{\dagger} d^{c} d^{c}, & D^{\mu} \chi^{\dagger} q^{\dagger} \bar{\sigma}_{\mu} d^{c}\end{array}$ & 5 & $\gg m_{\mathrm{Pl}}\left(g_{1}\right)$ \\
\hline 0 & $(6,2,5 / 6)$ & $1 / 3,4 / 3$ & $\begin{array}{c}\chi^{\dagger} H q q, \quad \chi H u^{c} u^{c}, \\
\chi H^{\dagger} u^{c} d^{c}, D^{\mu} \chi q^{\dagger} \bar{\sigma}_{\mu} u^{c}\end{array}$ & 5 & $\gg m_{\mathrm{Pl}}\left(g_{1}\right)$ \\
\hline 0 & $(\overline{6}, 2,7 / 6)$ & $2 / 3,5 / 3$ & $\chi^{\dagger} H d^{c} d^{c}$ & 5 & $\gg m_{\mathrm{Pl}}\left(g_{1}\right)$ \\
\hline 0 & $(8,1,0)$ & 0 & $\begin{array}{c}\chi H q u^{c}, \chi H^{\dagger} q d^{c}, \\
D^{\mu} \chi D^{\nu} G_{\mu \nu}, D^{\mu} \chi q^{\dagger} \bar{\sigma}_{\mu} q, \\
D^{\mu} \chi u^{c \dagger} \bar{\sigma}_{\mu} u^{c}, D^{\mu} \chi d^{c \dagger} \bar{\sigma}_{\mu} d^{c}, \\
\chi G^{\mu \nu} G_{\mu \nu}, \chi G^{\mu \nu} B_{\mu \nu}, \\
\chi \chi \chi H^{\dagger} H\end{array}$ & 5 & $\gg m_{\mathrm{Pl}}\left(g_{1}\right)$ \\
\hline 0 & $(8,1,1)$ & 1 & $\begin{array}{c}\chi H^{\dagger} q u^{c}, \chi^{\dagger} H q d^{c} \\
D^{\mu} \chi^{\dagger} u^{c \dagger} \bar{\sigma}_{\mu} d^{c}, \chi \chi \chi^{\dagger} H^{\dagger} H^{\dagger}\end{array}$ & 5 & $\gg m_{\mathrm{Pl}}\left(g_{1}\right)$ \\
\hline 0 & $(8,3,0)$ & 0,1 & $\begin{array}{c}\chi H q u^{c}, \chi H^{\dagger} q d^{c}, \\
\chi G^{\mu \nu} W_{\mu \nu}, D^{\mu} \chi q^{\dagger} \bar{\sigma}_{\mu} q, \\
\chi \chi \chi H^{\dagger} H\end{array}$ & 5 & $\gg m_{\mathrm{Pl}}\left(g_{1}\right)$ \\
\hline 0 & $(8,3,1)$ & $0,1,2$ & $\chi H^{\dagger} q u^{c}, \chi^{\dagger} H q d^{c}, \chi \chi \chi^{\dagger} H^{\dagger} H^{\dagger}$ & 5 & $1.0 \times 10^{17}\left(g_{1}\right)$ \\
\hline $1 / 2$ & $(6,1,1 / 3)$ & $1 / 3$ & $\chi^{c} \sigma^{\mu \nu} d^{c} G_{\mu \nu}$ & 5 & $\gg m_{\mathrm{Pl}}\left(g_{1}\right)$ \\
\hline $1 / 2$ & $(\overline{6}, 1,2 / 3)$ & $2 / 3$ & $\chi \sigma^{\mu \nu} u^{c} G_{\mu \nu}$ & 5 & $\gg m_{\mathrm{Pl}}\left(g_{1}\right)$ \\
\hline $1 / 2$ & $(8,1,1)$ & 1 & $\chi^{c} \sigma^{\mu \nu} e^{c} G_{\mu \nu}$ & 5 & $4.0 \times 10^{16}\left(g_{1}\right)$ \\
\hline
\end{tabular}

Table 2. Same as in table 1 but for colored states. 
multiplets at the renormalizable level. If these are charge- and color-neutral, they can form viable dark matter candidates, a possibility, which has been throughly investigated in the literature [4-7]. On the other hand, scenarios where the lightest component of the new multiplet is charged and/or colored are in general constrained by cosmological observations as well as by searches for exotic forms of matter on Earth and in the Universe. Taking also these constraints into account, the final list of viable uncolored and colored weak representations are given in tables 1 and 2, respectively, which summarize the main results of our investigation.

The details of the above sketched construction and analysis are contained in the rest of the paper which is organized as follows. In section 2 we exploit accidental symmetries beyond the SM to construct SM extensions with new degrees of freedom at the weak scale, which are completely transparent to indirect low-energy probes. The way the set of all possible extra states is made finite is discussed in this section as well. In section 3 we estimate the new particles' lifetimes. In turn in section 4 we consider bounds on possibly long lived states coming from early Universe cosmology. In particular, the effects on big bang nucleosynthesis (BBN) turn out to be the most important ones. Section 5 explores the collider phenomenology of the viable weak-scale SM extensions and estimates the current lower bounds on the new particles' masses coming from existing LHC searches. We conclude in section 6 while a more detailed technical discussion of the renormalization group (RG) evolution of the gauge couplings and the $\mathrm{SU}(2)_{L}$ decomposition of the effective operators are relegated to the appendices.

\section{Accidentally safe extensions of the SM}

Our starting point is the classification of SM extensions which automatically preserve the accidental and approximate symmetry structure of the SM without imposing additional protective mechanisms (only SM gauge and Lorentz symmetries are required). For simplicity, we will limit our discussion to the case where a single extra representation $\chi$ is added to the SM field content. While simultaneously adding more than one representation from our set is in principle possible, two additional restrictions need to be considered in that case: (i) adding more matter representations will in general lower the scale of the EFT validity (cf. section 2.4), (ii) additional SM gauge invariants may be constructed, potentially breaking $\mathcal{G}_{F}$ and/or the new accidental symmetry associated with $\chi$ stability at the renormalizable level.

We start by listing all the $d \leq 3$ operators made of SM fields. If $\chi$ is a fermion, we require that the new state does not couple to SM fermions at the renormalizable level. In this way, $\mathcal{G}_{F}$ is automatically preserved and an extra accidental symmetry guarantees the stability of the new particle at the renormalizable level. On the other hand, the case of extra scalars is more involved since they can always couple to the Higgs field at the renormalizable level without breaking $\mathcal{G}_{F}$ and their stability depends on the allowed interactions with the Higgs field.

A brief comment regarding larger Lorentz group representations is in order at this point. The presence of extra Lorentz vectors (i.e. spin 1 bosons) requires either the ex- 


\begin{tabular}{|c|c|c|c|c|}
\hline Spin & SM field & $\mathrm{SU}(3)_{c}$ & $\mathrm{SU}(2)_{L}$ & $\mathrm{U}(1)_{Y}$ \\
\hline 0 & $H$ & 1 & 2 & $+1 / 2$ \\
$1 / 2$ & $q$ & 3 & 2 & $+1 / 6$ \\
$1 / 2$ & $u^{c}$ & $\overline{3}$ & 1 & $-2 / 3$ \\
$1 / 2$ & $d^{c}$ & $\overline{3}$ & 1 & $+1 / 3$ \\
$1 / 2$ & $\ell$ & 1 & 2 & $-1 / 2$ \\
$1 / 2$ & $e^{c}$ & 1 & 1 & +1 \\
\hline
\end{tabular}

Table 3. SM field content and quantum numbers.

tension of the SM gauge group or new strong dynamics. In the former case, accidental preservation of $\mathcal{G}_{F}$ requires the extended gauge symmetry to be a direct product of the SM gauge group and possible new factors under which the SM fermions need to transform trivially. Such setups have been thoroughly studied in the literature (cf. [8] for a recent review) and we have nothing to add. On the other hand, new vectors due to some strong dynamics at the $\mathrm{TeV}$ scale are incompatible with a large mass gap $\Lambda_{\text {eff }} \gg \mathrm{TeV}$. The same argument applies to composite particles of higher spins. Finally, extra fundamental particles with spins $3 / 2$ and 2 can appear in theories of extended and gauged space-time symmetry (cf. $[9,10]$ ), but such constructions necessarily go beyond our EFT framework. We will hence limit our discussion to the inclusion of either spin 0 or $1 / 2$ extra representations.

In the following, we adopt a two-component notation where all the fermion fields are Weyl spinors belonging to the same irreducible representation of the Lorentz group. The accidental matter multiplets are collectively denoted by $\chi$. We use the subscripts $S$ and $F$ to denote the bosonic (spin 0) and fermionic (spin 1/2) SM gauge representations, respectively, where appropriate to avoid ambiguity. The SM fermions are collectively denoted by $\psi_{\mathrm{SM}}$ and their quantum numbers are fixed according to table 3 . The list of all possible $d \leq 3$ operators made of SM fields is provided in table 4.

\section{$2.1 \quad$ New fermions}

If a fermionic $\chi$ transforms under a complex or pseudoreal representation of the gauge group (so that a Majorana mass term is forbidden), we introduce another field $\chi^{c}$ with conjugate quantum numbers. In this way, the new state is vector-like and a mass term can always be added.

According to our previous discussion, we want to forbid the interactions $\chi \psi_{\mathrm{SM}}, \chi \psi_{\mathrm{SM}} H$ and $\chi \psi_{\mathrm{SM}} H^{\dagger} .^{2}$ By inspecting table 4 we conclude that $\chi$ cannot have the following quantum numbers:

$$
\chi \neq \psi_{\mathrm{SM}},(1,1,0),(1,3,0),(1,3,1),(1,2,3 / 2),(\overline{3}, 2,5 / 6),(3,2,7 / 6),(\overline{3}, 3,1 / 3),(3,3,2 / 3) .
$$

If $\chi$ transforms under a real representations of the SM group, then we can also add a Majorana mass term and the most general Langragian reads (see e.g. [11] for two-component

\footnotetext{
${ }^{2}$ Notice that terms of the form $\chi \chi H$ or $\chi \chi H^{\dagger}$ are forbidden by $\mathrm{SU}(2)_{L}$ invariance.
} 


\begin{tabular}{|c|c|c|c|c|}
\hline & $\mathcal{O}_{\mathrm{SM}}$ & $\mathrm{SU}(3)_{c}$ & $\mathrm{SU}(2)_{L}$ & $\mathrm{U}(1)_{Y}$ \\
\hline \multirow{5}{*}{$\psi_{\mathrm{SM}} H\left(H^{\dagger}\right)$} & $q H\left(H^{\dagger}\right)$ & 3 & $1 \oplus 3$ & $+2 / 3(-1 / 3)$ \\
\hline & $u^{c} H\left(H^{\dagger}\right)$ & $\overline{3}$ & 2 & $-1 / 6(-7 / 6)$ \\
\hline & $d^{c} H\left(H^{\dagger}\right)$ & $\overline{3}$ & 2 & $+5 / 6(-1 / 6)$ \\
\hline & $\ell H\left(H^{\dagger}\right)$ & 1 & $1 \oplus 3$ & $0(-1)$ \\
\hline & $e^{c} H\left(H^{\dagger}\right)$ & 1 & 2 & $+3 / 2(+1 / 2)$ \\
\hline \multirow{15}{*}{$\psi_{\mathrm{SM}} \psi_{\mathrm{SM}}$} & $q q$ & $\overline{3} \oplus 6$ & $1 \oplus 3$ & $+1 / 3$ \\
\hline & $q u^{c}$ & $1 \oplus 8$ & 2 & $-1 / 2$ \\
\hline & $q d^{c}$ & $1 \oplus 8$ & 2 & $+1 / 2$ \\
\hline & $q \ell$ & 3 & $1 \oplus 3$ & $-1 / 3$ \\
\hline & $q e^{c}$ & 3 & 2 & $+7 / 6$ \\
\hline & $u^{c} u^{c}$ & $3 \oplus \overline{6}$ & 1 & $-4 / 3$ \\
\hline & $u^{c} d^{c}$ & $3 \oplus \overline{6}$ & 1 & $-1 / 3$ \\
\hline & $u^{c} \ell$ & $\overline{3}$ & 2 & $-7 / 6$ \\
\hline & $u^{c} e^{c}$ & $\overline{3}$ & 1 & $+1 / 3$ \\
\hline & $d^{c} d^{c}$ & $3 \oplus \overline{6}$ & 1 & $+2 / 3$ \\
\hline & $d^{c} \ell$ & $\overline{3}$ & 2 & $-1 / 6$ \\
\hline & $d^{c} e^{c}$ & $\overline{3}$ & 1 & $+4 / 3$ \\
\hline & $\ell \ell$ & 1 & $1 \oplus 3$ & -1 \\
\hline & $\ell e^{c}$ & 1 & 2 & $+1 / 2$ \\
\hline & $e^{c} e^{c}$ & 1 & 1 & +2 \\
\hline \multirow{4}{*}{$\begin{array}{c}H \\
\text { combinations }\end{array}$} & $H H$ & 1 & 3 & +1 \\
\hline & $H H^{\dagger}$ & 1 & $1 \oplus 3$ & 0 \\
\hline & $H H H$ & 1 & 4 & $+3 / 2$ \\
\hline & $H H H^{\dagger}$ & 1 & $2 \oplus 4$ & $+1 / 2$ \\
\hline
\end{tabular}

Table 4. List of all possible $d \leq 3$ operators made of SM fields. Operators of the type $\psi_{\mathrm{SM}}^{\dagger} \psi_{\mathrm{SM}}$ are not displayed since they couple to Lorentz vectors, which are not considered in our analysis.

notation)

$$
\mathcal{L}=\mathcal{L}_{\mathrm{SM}}+i \chi^{\dagger} \bar{\sigma}^{\mu} D_{\mu} \chi+\frac{1}{2} M\left(\chi^{T} \epsilon \chi+\text { h.c. }\right),
$$

which is invariant under a $Z_{2}$ transformation $\chi \rightarrow-\chi$. On the other hand, if $\chi$ transforms under a complex or pseudoreal representations of the SM group, we introduce an extra Weyl fermion $\chi^{c}$ with conjugate gauge quantum numbers with respect to $\chi$, so that a Dirac mass term is allowed, and get

$$
\mathcal{L}=\mathcal{L}_{\mathrm{SM}}+i \chi^{\dagger} \bar{\sigma}^{\mu} D_{\mu} \chi+i \chi^{c \dagger} \bar{\sigma}^{\mu} D_{\mu} \chi^{c}+M\left(\chi^{T} \epsilon \chi^{c}+\text { h.c. }\right)
$$




\begin{tabular}{|c|c|c|c|c|}
\hline Spin & $\chi$ & $\mathcal{O}_{\text {decay }}$ & $\operatorname{dim}\left(\mathcal{O}_{\text {decay }}\right)$ & Stability \\
\hline 0 & $(1,1,0)$ & $\chi H H^{\dagger}$ & 3 & $\times$ \\
0 & $(1,3,0)$ & $\chi H H^{\dagger}$ & 3 & $\times$ \\
0 & $(1,4,1 / 2)$ & $\chi H H^{\dagger} H^{\dagger}$ & 4 & $\times$ \\
0 & $(1,4,3 / 2)$ & $\chi H^{\dagger} H^{\dagger} H^{\dagger}$ & 4 & $\times$ \\
0 & $(R, 2 k, 1 / 2)$ & $\chi \chi H^{\dagger} H^{\dagger}$ & 4 & $Z_{2}$ \\
0 & $(R, n, 0)$ & $\chi \chi H H^{\dagger}$ & 4 & $Z_{2}$ \\
0 & $(C, n, Y)$ & $\chi \chi^{\dagger} H H^{\dagger}$ & 4 & $\mathrm{U}(1)$ \\
0 & $(C, 2 k, 1 / 6)$ & $\chi \chi \chi H^{\dagger}$ & 4 & $Z_{3}$ \\
0 & $(R, 2 k, 1 / 2)$ & $\chi \chi \chi^{\dagger} H^{\dagger}$ & 4 & $\times$ \\
\hline
\end{tabular}

Table 5. Extra scalar representations which can couple to the Higgs at the renormalizable level without breaking $\mathcal{G}_{F}$. $(C, n, Y)$ denote generic quantum numbers under the SM gauge group which are not already contained in the list of eq. (2.4). $R$ stands for a real $\mathrm{SU}(3)_{c}$ representation (i.e. $R=$ $1,8,27, \ldots)$ and $2 k$ for an even $\mathrm{SU}(2)_{L}$ representation. In the last column, we provide (when appropriate) the symmetry responsible for the stability of $\chi$. The cases denoted by a " $\chi$ " lead instead to the decay of $\chi$ at the renormalizable level.

which is invariant under a $\mathrm{U}(1)$ transformation $\chi \rightarrow e^{i \theta} \chi$ and $\chi^{c} \rightarrow e^{-i \theta} \chi^{c}$. In both cases an accidental symmetry implies stability of the new particles at the renormalizable level and also requires that they are pair produced in high-energy particle colliders.

\subsection{New scalars}

For scalar $\chi$, in order to preserve $\mathcal{G}_{F}$ we have to avoid all couplings of the form $\chi \psi_{\mathrm{SM}} \psi_{\mathrm{SM}}$. By inspecting table 4 we conclude that $\chi$ cannot have the following quantum numbers:

$$
\begin{aligned}
\chi \neq & (1,1,1),(1,3,1),(1,1,2),(1,2,1 / 2),(\overline{3}, 1,1 / 3),(3,1,2 / 3),(\overline{3}, 1,4 / 3),(3,2,1 / 6), \\
& (3,2,7 / 6),(\overline{3}, 3,1 / 3),(6,1,1 / 3),(\overline{6}, 1,2 / 3),(6,1,4 / 3),(6,3,1 / 3),(8,2,1 / 2) .
\end{aligned}
$$

Analogously to the case of extra fermions in section 2.1, gauge interactions alone cannot lead to the decay of $\chi$ at the renormalizable level, since the kinetic terms again exhibit a $Z_{2}$ or a $\mathrm{U}(1)$ invariance for the case of an extra real or complex scalar, respectively. The decay of the new particle is however possible (depending on the quantum numbers of $\chi$ ) due to the presence of extra renormalizable interactions between $\chi$ and $H$, which are listed in table 5 .

\subsubsection{Scalar potential, CP and custodial symmetry}

In the presence of any new scalar multiplet $\chi$ the scalar potential can be written as (see e.g. [12])

$$
\begin{aligned}
V(H, \chi)=V_{\mathrm{SM}}+\eta\left(m_{\chi}^{2}|\chi|^{2}+\alpha|\chi|^{2}\right. & \left.|H|^{2}+\beta\left(\chi^{\dagger} T_{\chi}^{a} \chi\right)\left(H^{\dagger} T_{H}^{a} H\right)\right) \\
+ & {\left[\gamma\left(\chi^{\dagger} C_{\chi} T_{\chi}^{a} \chi^{*}\right)\left(H^{T} C_{H} T_{H}^{a} H\right)+\text { h.c. }\right]+\ldots, }
\end{aligned}
$$




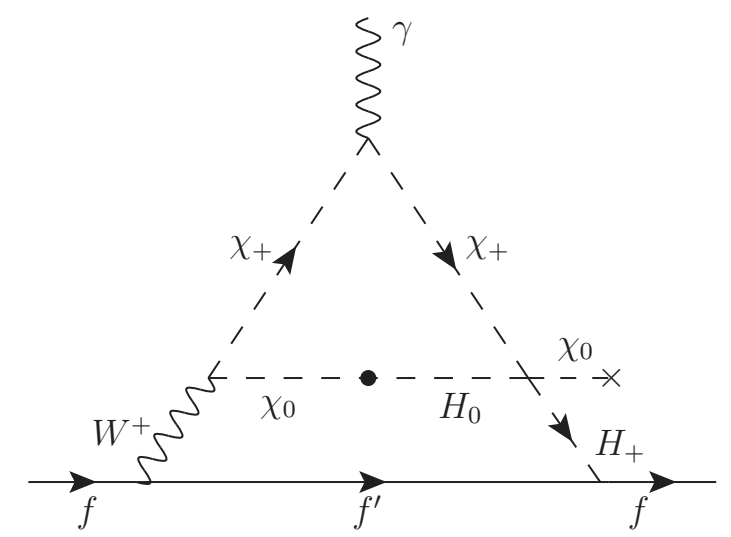

Figure 1. Scalar loop contribution to the electron EDM.

where $\eta$ is equal to $1(1 / 2)$ for a complex (real) representation, $T_{R}^{a}$ and $C_{R}$ denote respectively the $\mathrm{SU}(2)_{L}$ generators and conjugation matrices in the representation $R$ (so, for instance, $T_{H}^{a}=\sigma^{a} / 2$ and $C_{H}=i \sigma^{2}$ where $\sigma^{a}$ for $a=1,2,3$ are the Pauli matrices). We take $\langle H\rangle^{T}=(0, v / \sqrt{2})$ with $v=246 \mathrm{GeV}$. The ellipses in eq. (2.5) stand for extra terms, like e.g. $\left(\chi^{\dagger} T_{\chi}^{a} \chi\right)^{2}$, which do not sizeably affect the mass splitting of $\chi$ (see below). In addition, $\chi$ in specific weak representations might allow for additional renormalizable operators listed in table 5 .

The first accidental symmetry of the scalar potential that we wish to discuss is CP. Generic sources of $\mathrm{CP}$ violation are severely constrained by the measurement of electric dipole moments (EDMs) [13]. Among the accidental scalar matter extensions of tables 1-2, it turns out that only $(1,4,1 / 2)_{S}$ explicitly violates CP. This can be seen by noticing that for such a multiplet one can construct three non-hermitian invariants in the scalar potential (cf. the third, fifth and last row in table 5) and that only one out of the three phases associated with the corresponding complex couplings can be rotated away by a re-phasing of $\chi$ and $H$. In this case the most significant experimental constraint comes from the searches for an electron $\operatorname{EDM}\left(d_{e}\right)$, defined through the effective operator $\mathcal{L} \ni-i\left(d_{e} / 2\right) \bar{e}(\sigma \cdot F) \gamma_{5} e$. The $(1,4,1 / 2)_{S}$ contributes at two loops through the diagram in figure 1, which corresponds to the diagram in figure 12 of ref. [14] after replacing $H_{1} \rightarrow H$ and $H_{2} \rightarrow \chi$.

Taking into account the extra $\langle\chi\rangle / v \lesssim 1 \%$ suppression due to EW precision constraints (see below), assuming $\mathcal{O}(1)$ scalar couplings and mixing angles, and $m_{\chi} \sim v$ for the sake of a very conservative estimate (see also $[14,15]$ ), we obtain $\left|d_{e}\right| \lesssim 7 \times 10^{-29} e \mathrm{~cm}$. This has to be compared with the recent experimental bound from the ACME collaboration [16] of $\left|d_{e}^{\exp }\right|<8.7 \times 10^{-29} e \mathrm{~cm}$ at $90 \%$ C.L.. While not constraining at the moment, interestingly, future experimental improvements on the electron EDM might start to probe CP violation in generic weak-scale scalar extensions of the SM involving the $(1,4,1 / 2)_{S}$ multiplet.

Another accidental symmetry of the SM scalar potential is the so-called custodial symmetry. In the $g^{\prime} \rightarrow 0$ limit the massive gauge bosons transform as a triplet of an unbroken global $\mathrm{SU}(2)_{C}$, which is also responsible for the tree-level relation $\rho_{\text {tree }} \equiv$ $m_{W}^{2} / m_{Z}^{2} \cos ^{2} \theta_{W}=1$. New sources of $\mathrm{SU}(2)_{C}$ breaking which cannot be accounted 
in the $\mathrm{SM}$ are described by the $\rho_{0} \equiv \rho / \rho_{\mathrm{SM}}$ parameter [17]. Experimentally, $\rho_{0}^{\exp }=$ $1.0004_{-0.0004}^{+0.0003}$ [17], which is compatible with the SM prediction $\rho_{0}=1$. Thus the experimental value of $\rho_{0}$ can be used to constrain new sources of $\mathrm{SU}(2)_{C}$ breaking due to the extra scalar $\chi$.

If $\chi$ gets a vacuum expectation value (VEV), there is a tree-level contribution [18]

$$
\rho_{0}^{\text {tree }}-1=\left\{\eta\left[j(j+1)-Y^{2}\right]-2 Y^{2}\right\}\left[4 \frac{\langle\chi\rangle^{2}}{v^{2}}+\mathcal{O}\left(\frac{\langle\chi\rangle^{4}}{v^{4}}\right)\right],
$$

where $j$ is the total weak-isospin quantum number of $\chi$ and $Y$ its hypercharge in the $Q=T^{3}+Y$ normalization. Apart for the safe representations yielding $\rho_{0}=1$ for any value of $\langle\chi\rangle:(1,1,0),(1,2,1 / 2),(1,7,2)[19],(1,26,15 / 2)$, etc., the $2 \sigma$-level saturated bound is at the level of $\langle\chi\rangle / v \lesssim 1 \%$.

In general, whether a scalar field can develop a VEV depends on the choice of the parameters in the scalar potential. However, "tadpole" couplings of $\chi$ to some $H$ 's always imply an induced VEV for $\chi$. From table 5 we see that this is indeed the case for the states: $(1,1,0),(1,3,0),(1,4,1 / 2),(1,4,3 / 2)$. While the VEV of the former does not contribute to $\rho_{0}$, the remaining ones can be in principle dangerous. By looking at the generic shape of the potential and its stationary equations, we estimate on dimensional grounds (for $\mathcal{O}(1)$ couplings and barring fine-tunings), $\langle\chi\rangle \sim v^{2} / m_{\chi}$ (triplet case) and $\langle\chi\rangle \sim v^{3} / m_{\chi}^{2}$ (quadruplet cases). Hence, $\langle\chi\rangle / v \lesssim 1 \%$ corresponds to $m_{\chi} \gtrsim 100 v \approx 20 \mathrm{TeV}$ (triplet case) and $m_{\chi} \gtrsim 10 v \approx 2 \mathrm{TeV}$ (quadruplet cases), which limits the visibility of these states at the LHC, unless a moderate fine-tuning is allowed in the scalar potential.

Custodial symmetry also helps us to understand the properties of the theory beyond the tree level. Indeed, a tree-level splitting within the components of $\chi$ originating from the scalar potential in eq. (2.5) gives a radiative contribution to $\rho_{0}$. In the following we assume $\langle\chi\rangle \ll v$ to suppress the tree-level contribution to $\rho_{0}$. Consequently $\langle\chi\rangle$ itself cannot sizably contribute to the mass splitting. Notice, also, that among the scalar states selected in table 1 , the coupling $\gamma$ is relevant only for $(1,4,1 / 2)$. However, since this state decays through a renormalizable operator, the details of its mass spectrum are not of particular interest. ${ }^{3}$ We are hence left with the contribution of $\beta$ to the mass splitting, which yields

$$
m_{I}^{2}=m_{\chi}^{2}+\frac{1}{2} \alpha v^{2}-\frac{1}{4} \beta v^{2} I \equiv M^{2}-\delta^{2} I
$$

where $-j \leq I \leq j$ denotes the $T^{3}$ eigenvalue of the $(2 j+1)$-dimensional representation $\chi$ and we defined the parameter $M^{2} \equiv m_{\chi}^{2}+\frac{1}{2} \alpha v^{2}$ and $\delta^{2} \equiv \frac{1}{4} \beta v^{2}$. Using the general formula for the one-loop correction in [20] and expanding the loop function for $\delta<M$ we find

$$
\rho_{0}^{1-\text { loop }}-1=\frac{\eta N_{C} \alpha_{\mathrm{em}}}{16 \pi \sin ^{2} \theta_{W} m_{W}^{2}}\left[\frac{2}{9} \frac{\delta^{4}}{M^{2}} j(j+1)(2 j+1)+\mathcal{O}\left(\frac{\delta^{8}}{M^{6}}\right)\right],
$$

\footnotetext{
${ }^{3}$ The coupling $\gamma$ induces mixing between the conjugate components of $\chi$ with the same $|Q| \neq 0$ and, for the $Q=0$ component, it splits its real and imaginary part. The contribution of $\gamma$ to $\rho_{0}^{1-\text { loop }}$ has been considered for instance in [12].
} 
where $N_{C}$ is the dimensionality of $\chi$ under the color factor. Neglecting the higher-order $\delta / M$ terms, we finally obtain

$$
M \gtrsim 72.5 \mathrm{GeV}\left(\frac{0.001}{\rho_{0}^{\exp }-1}\right)^{1 / 2} \beta \sqrt{\eta N_{C}} \sqrt{j(j+1)(2 j+1)},
$$

which is valid for $M>\delta \approx \sqrt{\beta} 123 \mathrm{GeV}$. For $\mathcal{O}(1)$ values of the coupling $\beta$ the typical bounds on $M$ range in the few hundred $\mathrm{GeV}$ region, depending on the dimensionality of the representation. We hence conclude that the mass bounds coming from loop-level contributions to $\rho_{0}$ are less general (they depend on the value of $\beta$ ) and not particularly constraining when compared to existing direct searches limits (see section 5). This is, however, not necessarily true for higher dimensional representations.

Alternatively, the information from $\rho_{0}$ can be used to give an upper bound on the mass splitting $\Delta m=m_{I+1}-m_{I} \approx-\frac{\delta^{2}}{2 M}$. As an example, let us mention that for the case $(1,5,2)_{S}$ we get $\Delta m \lesssim 20 \mathrm{GeV}$. This information is exploited in section 5.3 when inferring collider bounds on the neutral state of such a multiplet by looking at the charged component production and decays.

\subsubsection{Bounds on Higgs portal coupling}

The Higgs boson can couple to the new scalars via the portal coupling $\alpha$ of eq. (2.5). This leads to two kinds of effects: (1) If some components of $\chi$ lie below half of the Higgs mass, they can contribute to the Higgs total decay width. Taking into account other existing collider constraints (see table 8) this is only possible for the neutral component $\chi_{0}$. In particular, it contributes to the Higgs invisible decay branching fraction. The partial decay width of the Higgs boson via the $\alpha$ coupling into a pair of $\chi_{0}$ states (for $\beta=0$ ) is found to be

$$
\Gamma_{\mathrm{inv}}=\frac{\eta \alpha^{2} v^{2}}{16 \pi} \frac{1}{m_{H}} \sqrt{1-\frac{4 m_{\chi_{0}}^{2}}{m_{H}^{2}}}
$$

In addition, (2) all charged components of $\chi$ will contribute at 1-loop level to the $H \rightarrow \gamma \gamma$ (and $H \rightarrow \gamma Z$ ) decays, while colored $\chi$ will affect Higgs boson production through gluon fusion (GF) and also its decays to two gluons $H \rightarrow g g$. Using the results of [21-23] we find

$$
\begin{aligned}
& \mu_{\gamma \gamma} \equiv \frac{\Gamma_{\gamma \gamma}}{\Gamma_{\gamma \gamma}^{\mathrm{SM}}}=\frac{\left|\mathcal{A}_{1}\left(x_{W}\right)+(4 / 3) \mathcal{A}_{1 / 2}\left(x_{t}\right)+\eta \alpha d\left(R_{\chi}\right) \sum_{i} Q_{i}^{2}\left(v / m_{\chi_{i}}\right)^{2} \mathcal{A}_{0}\left(x_{\chi_{i}}\right)\right|^{2}}{\left|\mathcal{A}_{1}\left(x_{W}\right)+(4 / 3) \mathcal{A}_{1 / 2}\left(x_{t}\right)\right|^{2}}, \\
& \mu_{g g} \equiv \frac{\Gamma_{g g}}{\Gamma_{g g}^{\mathrm{SM}}}=\frac{\sigma_{\mathrm{GF}}}{\sigma_{\mathrm{GF}}^{\mathrm{SM}}}=\frac{\left|(1 / 2) \mathcal{A}_{1 / 2}\left(x_{t}\right)+\eta \alpha C\left(R_{\chi}\right) \sum_{i}\left(v / m_{\chi_{i}}\right)^{2} \mathcal{A}_{0}\left(x_{\chi_{i}}\right)\right|^{2}}{\left|(1 / 2) \mathcal{A}_{1 / 2}\left(x_{t}\right)\right|^{2}}
\end{aligned}
$$

where $x_{i} \equiv m_{H}^{2} / 4 m_{i}^{2}$, the sums $\sum_{i}$ run over all $\chi$ weak multiplet components $\chi_{i}, d\left(R_{\chi}\right)$ is the dimension of the color representation of $\chi$ and $C\left(R_{\chi}\right)$ is the corresponding index $(C(3)=1 / 2, C(6)=5 / 2$ and $C(8)=3)$. The relevant loop functions $\mathcal{A}_{1}\left(x_{W}\right) \simeq-8.32$, $\mathcal{A}_{1 / 2}\left(x_{t}\right) \simeq 1.38$ and $A_{0}(x)$ with limits $A_{0}(x \rightarrow 0)=1 / 3, A_{0}(x \rightarrow \infty)=-1 / x+\mathcal{O}\left(x^{-2}\right)$ can be found e.g. in [23]. The total decay width of the Higgs can thus be written as

$$
\Gamma_{H}=\Gamma_{H}^{\mathrm{SM}}\left[1+B R_{\gamma \gamma}^{\mathrm{SM}}\left(\mu_{\gamma \gamma}-1\right)+B R_{g g}^{\mathrm{SM}}\left(\mu_{g g}-1\right)\right]+\Gamma_{\mathrm{inv}},
$$




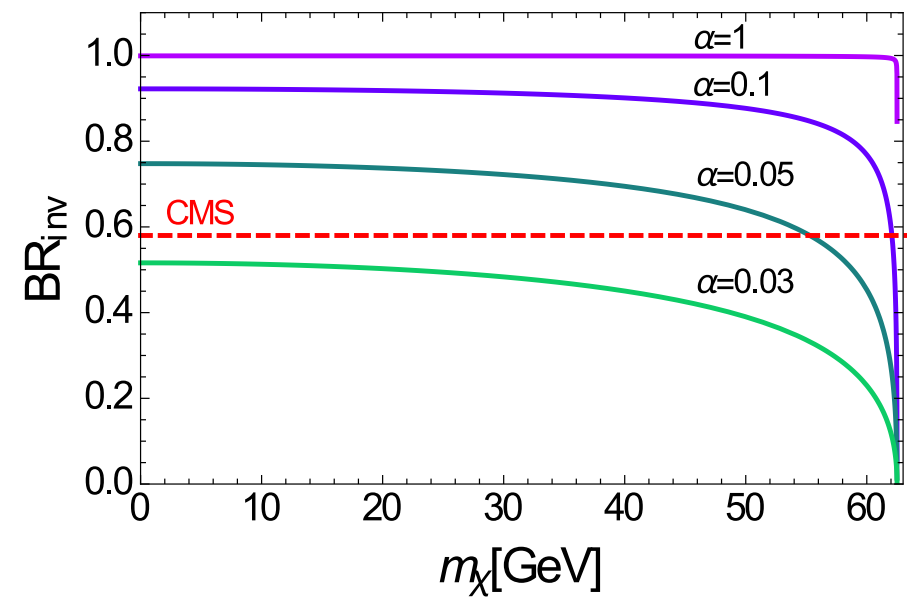

Figure 2. Invisible branching fraction of the Higgs boson as a function of the new scalars mass (real scalar) for different values of the portal coupling $\alpha$. The red dashed line shows the CMS exclusion limit of ref. [25].

where $\Gamma_{H}^{\mathrm{SM}}=4.07 \mathrm{MeV}, \mathrm{BR}_{\gamma \gamma}^{\mathrm{SM}}=2.28 \times 10^{-3}$ and $\mathrm{BR}_{g g}^{\mathrm{SM}}=8.57 \times 10^{-2}$ [24]. The invisible branching ratio is then finally given by $\mathrm{BR}_{\mathrm{inv}}=\Gamma_{\mathrm{inv}} / \Gamma_{H}$.

To analyze the resulting correlated effects in Higgs boson production and decays as measured at the LHC, we follow closely the procedure described in [26] using also the same set of experimental results [25, 27-36]. In particular, we find that in cases where the Higgs boson can decay to $\chi_{0}$, the constraints on $\alpha$ are completely dominated by the bounds on the extra invisible decay rate. In figure 2 the invisible branching ratio as a function of the new scalar mass $m_{\chi}$ is shown for different values of the portal coupling $\alpha$. We used $\eta=1 / 2$ in eq. (2.10), assuming a real scalar. The red dashed line shows the CMS limit of ref. [25]. ${ }^{4}$ It can be inferred from the plot that for a portal coupling $|\alpha|=\mathcal{O}(1)$, the new scalar states are excluded up to the kinematic limit for this decay. However for values of $|\alpha| \lesssim \mathcal{O}(0.01)$ currently no limit on $m_{\chi_{0}}$ can be given anymore.

Even if $\chi_{i}$ are heavy $\left(m_{\chi_{i}}>m_{H} / 2\right)$, their contributions to $\mu_{\gamma \gamma}$ and $\mu_{g g}$ still lead to constraints on $\alpha$ from the measurements of the Higgs signal strengths at the LHC. In particular, the most sensitive channels involve GF produced Higgs bosons decays to photons and $\mathrm{W}$ bosons, these being the two most precisely measured. Denoting the relevant signal strenghts as

$$
\mu_{\gamma \gamma}^{\mathrm{GF}} \equiv \frac{\sigma_{\mathrm{GF}}}{\sigma_{\mathrm{GF}}^{\mathrm{SM}}} \frac{\mathrm{BR}_{\gamma \gamma}}{\mathrm{BR}_{\gamma \gamma}^{\mathrm{SM}}}, \quad \mu_{W W}^{\mathrm{GF}} \equiv \frac{\sigma_{\mathrm{GF}}}{\sigma_{\mathrm{GF}}^{\mathrm{SM}}} \frac{\mathrm{BR}_{W W}}{\mathrm{BR}_{W W}^{\mathrm{SM}}},
$$

the global fit of Higgs boson LHC data allowing for arbitrary contributions to $\mu_{\gamma \gamma}$ and $\mu_{g g}$ but keeping $\Gamma_{\text {inv }}=0$ yields the $68 \%$ and $95 \%$ CL exclusion bounds shown in figure 3 . We observe that up to $50 \%$ modifications in both observables are still allowed by the current data. These should be compared with $\alpha$ induced modifications shown in figure 4 (assuming degenerate $\left.\chi_{i}\right)$. In particular, color-neutral $\chi$ predominantly affect $\mu_{\gamma \gamma}^{\mathrm{GF}}$ as shown in the left panel. On the other hand, colored states can affect GF production and are thus constrained

\footnotetext{
${ }^{4}$ Indirect bound on $\mathrm{BR}_{\text {inv }}$ coming from the global fit to all Higgs boson signal strenghts yields a slightly stronger bound of $\mathrm{BR}_{\text {inv }} \lesssim 0.2$.
} 


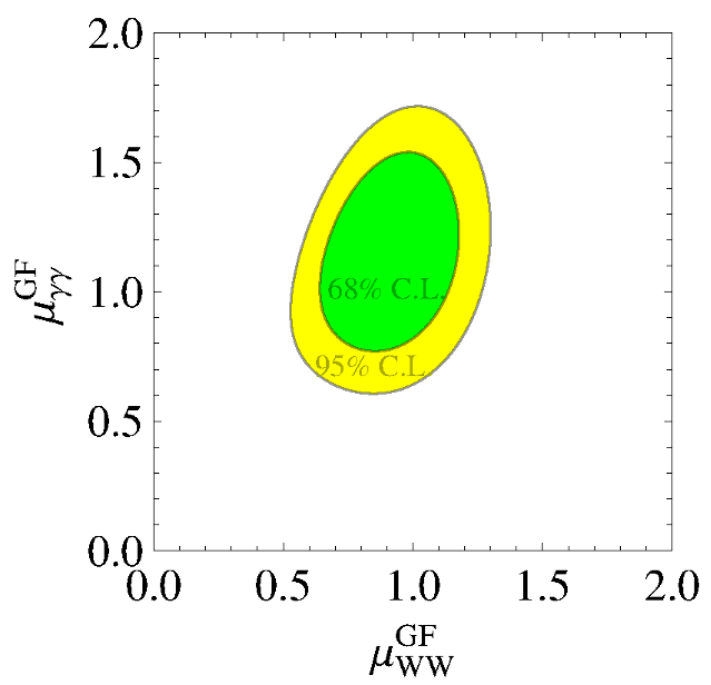

Figure 3. Exclusion bounds on the $\mu_{\gamma \gamma}^{\mathrm{GF}}$ and $\mu_{W W}^{\mathrm{GF}}$ LHC Higgs signal strengths allowing for arbitrary contributions to $\mu_{\gamma \gamma}$ and $\mu_{g g}$ but keeping $\Gamma_{\text {inv }}=0$.
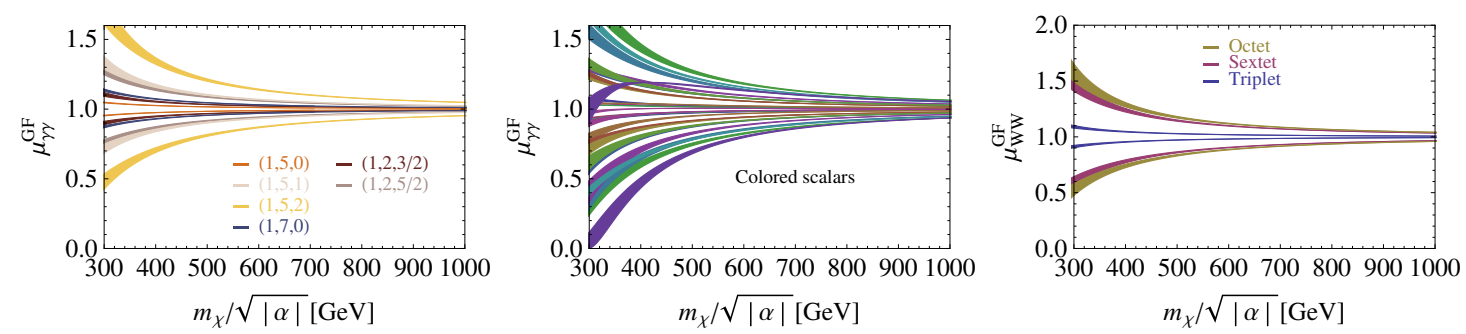

Figure 4. Accidental scalar induced modifications to $\mu_{\gamma \gamma}^{\mathrm{GF}}$ and $\mu_{W W}^{\mathrm{GF}}$ LHC Higgs signal strengths as a function of $m_{\chi} / \sqrt{|\alpha|}$. The un-colored and colored scalar effects in $\mu_{\gamma \gamma}^{\text {GF }}$ are shown in the left and middle panel, respectively. Single (complex) colored scalar effects in $\mu_{W W}^{\mathrm{GF}}$ are shown in the right panel. The shaded bands correspond to a scan $|\alpha| \in[0.1,1]$.

also from $\mu_{W W}^{\mathrm{GF}}$ as illustated in the right panel. The deviations in $\mu_{W W}^{\mathrm{GF}}$ are shown for single complex scalar in the given color representation. Finally, colored scalar effects in $\mu_{\gamma \gamma}^{\mathrm{GF}}$ are also shown in the middle panel.

Asymptotically, $\chi$ effects in both observables decouple as $\alpha / m_{\chi}^{2}$. The shaded bands in figure 4 illustrate the amount of deviations from this limit as they correspond to a scan of $|\alpha| \in[0.1,1]$. We observe that for $m_{\chi} \gtrsim 500 \mathrm{GeV}$ even $|\alpha| \lesssim \mathcal{O}(1)$ can be consistent with current Higgs data. Conversely $m_{\chi} \gtrsim 100 \mathrm{GeV}$ are perfectly allowed for small enough $|\alpha| \lesssim \mathcal{O}(0.1)$ Higgs portal couplings.

\subsection{Mass spectrum}

The phenomenology of the new EW states is dictated by the mass spectrum. Typically, on top of a common mass term $m_{\chi}$, there is a radiative splitting within the $\mathrm{SU}(2)_{L}$ multiplet and, for scalars only, a tree-level splitting due to the presence of non-trivial $\mathrm{SU}(2)_{L}$ invariants in the scalar potential. In the $m_{\chi} \gg v$ limit the radiative contribution takes the 
form $[4,37]$

$$
\Delta m_{\mathrm{rad}}=m_{Q+1}-m_{Q} \approx 166 \mathrm{MeV}\left(1+2 Q+\frac{2 Y}{\cos \theta_{W}}\right)
$$

which holds both for fermions and scalars. Notice that if $Y=0$ the LP in the multiplet is always the one with the smallest $|Q|$. This is not necessarily true when $Y \neq 0 .{ }^{5}$

Similarly, the tree-level splitting in eq. (2.7) can be expanded in the $m_{\chi} \gg v$ limit, thus obtaining [4]

$$
\Delta m_{\text {tree }}=m_{I+1}-m_{I} \approx \frac{\beta v^{2}}{8 m_{\chi}} \approx \beta \times 7.6 \mathrm{GeV}\left(\frac{1 \mathrm{TeV}}{m_{\chi}}\right) .
$$

Notice that, while for fermions the mass spectrum is unambiguously fixed, for scalars it depends on the values of $\beta$ and $m_{\chi}$. Focussing on the $m_{\chi}<\mathrm{TeV}$ region (relevant for LHC), if $\beta=\mathcal{O}(1)$ then the LP is always the one with the highest/lowest $I$, depending on the sign of $\beta$. However, for $\beta \in\left[10^{-3}, 1\right]$ the tree-level splitting can be comparable with the radiative one. In such cases it is possible to show (see below) that:

1. Any particle in the multiplet can be the LP for large domains of the model parameters, i.e. without any fine-tuning.

2. If the LP has charge $Q_{\mathrm{LP}}$, the next-to-LP has always charge $Q_{\mathrm{LP}} \pm 1$.

This latter fact turns out to be phenomenologically relevant, e.g. when setting bounds on the neutral LP by looking at the decay of the next-to-LP.

For completeness, we provide here a proof of the two statements above: by combining eq. (2.14) and eq. (2.15) one arrives at the expression $m_{I}=m_{-j}+a+b I+c I^{2}$, where $a$ and $b$ can have any sign (since they depend on $\Delta m_{\text {tree }}$ ), and $c>0$. The minimum of $m_{I}$ is obtained for $I_{\min }=-\frac{b}{2 c}$. Hence, by an appropriate choice of the ratio $b / c$, the smallest $m_{I}$ can be anywhere in the range $I \in[-j, j]$. The fact that the next-to-LP has $Q_{\mathrm{LP}} \pm 1$ simply follows from the convexity of $m_{I}$ as a function of $I$. A similar argument holds as well in the $m_{\chi} \approx v$ regime, for which the full formula of the radiative splitting (see e.g. eq. (6) in [4]) must be taken into account.

\subsection{Validity of the EFT}

Our working hypothesis is that the $\mathrm{SM}+\chi$ renormalizable theory is a low-energy effective description valid up to a cut-off scale $\Lambda_{\text {eff }}$. In the spirit of a generic EFT with $\mathcal{O}(1)$ couplings and without any extra state beyond $\chi$ introduced at low energy, $\Lambda_{\text {eff }} \approx 10^{15} \mathrm{GeV}$ is essentially fixed by neutrino masses through the $d=5$ Weinberg operator. Moreover, such a cut-off scale can automatically account for null results of all flavor, CP and B violating processes constraining $d=6$ operators made of SM fields. In particular, when the lowest-dimensional sources of breaking of the extra $\mathrm{U}(1)$ or $Z_{2}$ symmetry associated with the kinetic term of $\chi$ are the $d=5$ operators involving $\chi$ and SM fields, any $d=6$ operator involving only SM fields, generated by integrating out $\chi$, will have two insertions of such $d=5$ operators and hence at least a $1 / \Lambda_{\text {eff }}^{2}$ suppression. The situation changes only slightly if the extra $\mathrm{U}(1)$ or $Z_{2}$ is broken at the renormalizable level in the scalar potential,

\footnotetext{
${ }^{5}$ E.g. the LP of the fermion multiplet $(1,4,1 / 2)_{F}$ has $Q=-1$.
} 
as in cases listed in table 5. Namely, the only additional effect arises for $\chi \sim(1,4,1 / 2)_{S}$, $(1,4,3 / 2)_{S}$ where integrating out $\chi$ induces a $\Delta L=2$ operator of the form $\ell \ell H H H H^{\dagger}$. Being suppressed by $1 / \Lambda_{\text {eff }} m_{\chi}^{2}$, it necessarily represents a subleading contribution to neutrino masses.

The infinite set of states preserving $\mathcal{G}_{F}$ at the renormalizable level (see eq. (2.1) and eq. (2.4)) can be reduced by requiring that the EFT remains weakly coupled up to $\Lambda_{\text {eff }} \approx$ $10^{15} \mathrm{GeV}$. The presence of extra matter multiplets drives the gauge couplings of the SM towards the non-perturbative regime. ${ }^{6}$ Eventually, this might result in the presence of a Landau pole below the cut-off scale of the EFT. If the Landau pole is associated with a generic new dynamics, the accidental symmetries of the SM could be violated at that scale. Hence, for the self-consistency of the EFT approach, we require the absence of Landau poles below $\Lambda_{\text {eff }} \approx 10^{15} \mathrm{GeV}$, which translates into an upper bound on the dimensionality of the extra representations.

In light of stringent bounds on the inter-multiplet mass splittings (see section 2.3) we can safely integrate in all multiplet components at a single scale, which we choose to be the $Z$ mass in our numerical analysis. We note however, that for $m_{\chi}$ not much larger than the $\mathrm{TeV}$ scale the resulting Landau pole estimates scale linearly with $\chi$ masses. The analysis of the perturbativity bounds is detailed in appendix A and the results are summarized in tables 9-10. They provide a useful reference for the estimate of the Landau poles at two loops for the cases where the SM is extended with an extra multiplet charged under $\mathrm{SU}(3)_{c}$ and/or $\mathrm{SU}(2)_{L}$, and in particular for all the states considered in this work which can have a non-zero hypercharge as well.

A crucial ingredient in order to make our list of extra states finite however, is given by cosmology. In fact, the only reason why we can disregard multiplets with an arbitrary hypercharge, e.g. $Y=\pi$, is because these states feature an absolutely stable charged LP that cannot decay into SM particles because of electric charge conservation. The possibility of having an infinitesimal hypercharge is instead briefly discussed in section 4 .

A comment on the role of higher-order corrections in the RG equations is in order here. The determination of the Landau pole is often carried out at the one-loop level (see e.g. [4]). However, for the non-abelian gauge factors there is an accidental cancellation in the one-loop beta function between matter and gauge contributions (cf. eq. (A.2)), so that two-loop effects may become important. Interestingly, among the cases that we found to be drastically affected by two-loop corrections there are the two minimal DM candidates: a real $(1,7,0)$ scalar and a Weyl $(1,5,0)$ fermion [4]. ${ }^{7}$ Following the results of [5] for the calculation of the relic density, we integrate in the scalar septuplet at $m_{\chi}=25 \mathrm{TeV}$ and the fermionic quintuplet at $m_{\chi}=10 \mathrm{TeV}$. Hence we find, respectively

$$
\begin{array}{llll}
\Lambda_{\text {Landau }}^{1 \text {-loop }}=1.9 \times 10^{41} \mathrm{GeV} & \longrightarrow & \Lambda_{\text {Landau }}^{2 \text {-loop }}=8.9 \times 10^{20} \mathrm{GeV} & \left((1,7,0)_{S} \text { case }\right) \\
\Lambda_{\text {Landau }}^{1 \text {-loop }}=9.0 \times 10^{28} \mathrm{GeV} & \longrightarrow & \Lambda_{\text {Landau }}^{2 \text {-loop }}=4.0 \times 10^{21} \mathrm{GeV} & \left((1,5,0)_{F} \text { case }\right)
\end{array}
$$

\footnotetext{
${ }^{6}$ We do not address here the question of the RG running of the scalar potential parameters, since it is a model dependent issue which also involves the analysis of the vacuum stability.

${ }^{7}$ Another situation where the two-loop RG analysis of the gauge couplings could change the qualitative UV behaviour of the theory is given by the Pati-Salam model presented in [38], where low-scale extensions of the SM providing total asymptotic freedom are investigated.
} 
If we associate the Landau pole with the cut-off of a generic EFT, this also sets the scale of the effective operator leading to the decay of the minimal dark matter candidate. Note however, that even for a cut-off of the order of the Planck mass, the framework of minimal DM is not endangered by $d \geq 6$ operators, since the lifetime of DM is still comfortably larger than the age of the Universe (and satisfies the indirect bounds on decaying DM). For a discussion of $d=5$ induced $(1,7,0)_{S}$ decays see section 3.3.2.

In the selection of our states, the two-loop criterium proved to be important for several states. For instance, in the case of the real $(27,1,0)_{S}$ scalar multiplet we find that at one loop $\Lambda_{\text {Landau }}^{1 \text {-loop }}=1.9 \times 10^{41} \mathrm{GeV}$, whereas at the two-loop level $\Lambda_{\text {Landau }}^{2 \text {-loop }}=1.3 \times 10^{7} \mathrm{GeV}$, so that we can exclude this state from our list of accidental matter candidates.

What about three-loop corrections then? As long as there are no accidental cancellations in the two-loop beta function (as it can be explicitly verified), they are not expected to drastically change the situation. ${ }^{8}$ It is then enough to rely on a two-loop estimate of the Landau pole in order to set an upper bound on the dimensionality of the extra representation.

\section{Lifetimes}

The new extra states will eventually decay due to operators present in the EFT. There are essentially three classes of decays which we are going to consider in this section: i) Intermultiplet weak transitions where the heavier components within the $\mathrm{SU}(2)_{L}$ multiplet decay via cascades involving the emission of (virtual) $W$ gauge bosons into the LP, ii) Decays through renormalizable interactions (only for a specific class of new scalar states) and iii) Decays through non-renormalizable $d \geq 5$ operators. We analyze each class of decays in turn below.

\subsection{Inter-multiplet weak transitions}

Heavier components within the $\mathrm{SU}(2)_{L}$ multiplet can decay via EW transitions into lighter ones, with rates suppressed by a small phase space factor. Denoting the component of a total $j$-isospin representation with $T^{3}$-eigenvalue $I$ as $\chi_{I}^{j}$, for $\Delta m>m_{\pi^{+}}$, we have the decay width (generalizing the expression in ref. [37])

$$
\Gamma\left(\chi_{I+1}^{j} \rightarrow \chi_{I}^{j} \pi^{+}\right)=\frac{T_{+}^{2} G_{F}^{2} V_{u d}^{2} \Delta m^{3} f_{\pi^{+}}^{2}}{\pi} \sqrt{1-\frac{m_{\pi^{+}}^{2}}{\Delta m^{2}}} \approx \frac{T_{+}^{2}}{7.5 \times 10^{-12} \mathrm{~s}}\left(\frac{\Delta m}{500 \mathrm{MeV}}\right)^{3}
$$

where $T_{+}=\sqrt{j(j+1)-I(I+1)}$ and the approximation in the r.h.s. of eq. (3.1) is valid for $\Delta m \gg m_{\pi^{+}}$.

Formula (3.1) is a reasonable approximation of the total width in the range $m_{\pi^{+}} \lesssim$ $\Delta m \lesssim 1 \mathrm{GeV}$. For mass splittings close to the kinematical threshold of the decay into a pion, 3-body decays involving leptons become important as well, while for $\Delta m \gtrsim 1 \mathrm{GeV}$

\footnotetext{
${ }^{8}$ For instance, in the SM case where no strong cancellations are at play we find: $\Lambda_{\text {Landau }}^{1 \text {-loop }}=1.9 \times 10^{41} \mathrm{GeV}$, $\Lambda_{\text {Landau }}^{2 \text {-loop }}=5.2 \times 10^{40} \mathrm{GeV}$ and $\Lambda_{\text {Landau }}^{3 \text {-loop }}=8.7 \times 10^{40} \mathrm{GeV}$.
} 


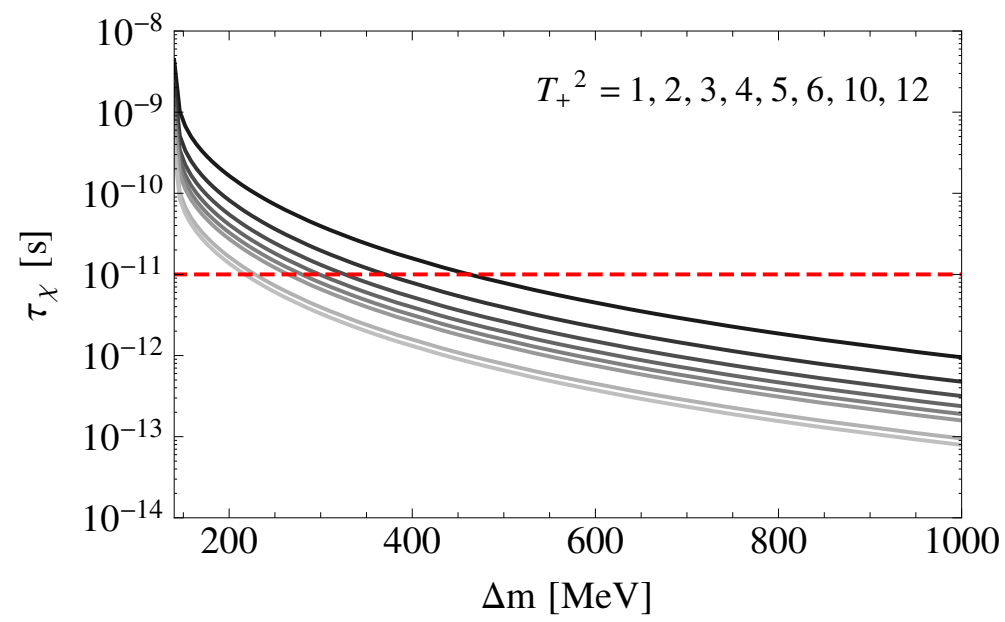

Figure 5. Lifetimes associated with inter-multiplet weak transitions as a function of the mass splitting $\Delta m$. The grading of the curves (from black to gray) corresponds to different values of $T_{+}^{2}$ (from 1 to 12), as it can be found in representations up to $j=3$ (septuplet). The red dashed line corresponds to the typical freeze-out time for colorless $1 \mathrm{TeV}$-mass particles with weak interactions in the early Universe (cf. section 4).

new hadronic channels open up (e.g. involving kaons and other heavier hadrons) and the decay can be eventually computed at the partonic level, once quark-hadron duality sets in.

The typical lifetime of an $\mathrm{SU}(2)_{L}$ multiplet component decaying via inter-multiplet weak transitions is displayed in figure 5 as a function of the mass splitting and for different values of the ladder operator $T_{+}$, up to the $j=3$ (septuplet) case.

Within high energy collider experiments, the inter-multiplet decays are essentially prompt. On the other hand, the LP at the end of these inter-multiplet cascades is stable on the detector scale, barring few exception which are discussed in the next subsection.

\subsection{Decays through renormalizable interactions}

There exists the possibility that the new extra scalars retain renormalizable interactions with the SM Higgs which can induce their decay. These states are classified in table 5 and correspond to the cases (labelled with the symbol " $\times$ ") where no accidental symmetry (e.g. $\mathrm{U}(1), Z_{2}$ or $Z_{3}$ ) forbids $\chi$ to decay. Let us comment in turn on the various possibilities.

The case of the gauge singlet $(1,1,0)_{S}$ has been extensively studied in the literature (see e.g. [39]) and we do not have much to add here. In the cases $(1,3,0)_{S},(1,4,1 / 2)_{S}$, and $(1,4,3 / 2)_{S}, \chi$ can couple linearly to Higgs operators. However, such "tadpole" couplings also induce non-zero VEVs for $\chi$, which are severely constrained by EW precision observables. As already pointed out in section 2.2.1, unless a moderate fine-tuning is allowed in the scalar potential, the bounds on such dimensionally estimated VEVs push the masses of these states beyond the kinematic reach of the LHC. Other multiplets which can possibly decay at the renormalizable level (those labelled with a " $\times$ " in table 5) are either not considered here because they break $\mathcal{G}_{\mathcal{F}}$ by coupling to SM fermions (e.g. $(8,2,1 / 2)_{S}$ ) or they generate a Landau pole below $\Lambda_{\text {eff }} \approx 10^{15} \mathrm{GeV}$ (e.g. $\left.(1,6,1 / 2)_{S}\right)$. 


\subsection{Decays through $d \geq 5$ effective operators}

Let us consider now the case where the decay of the new state $\chi$ is due to effective operators. Given an effective operator $\mathcal{O}_{\text {decay }}$, we always absorb the Wilson coefficient in the definition of the effective cut-off scale $\Lambda_{\text {eff }}$, e.g.

$$
\mathcal{L} \ni \frac{1}{\Lambda_{\text {eff }}} \mathcal{O}_{\text {decay }}+\text { h.c. . }
$$

The differential decay rate of an unstable particle $\chi$ into $n_{f}$ final states reads

$$
d \Gamma=\frac{1}{2 m_{\chi}}\left(\prod_{f} \frac{d^{3} p_{f}}{2 \pi^{3}} \frac{1}{2 E_{f}}\right)\left|\mathcal{M}\left(m_{\chi} \rightarrow\left\{p_{f}\right\}\right)\right|^{2}(2 \pi)^{4} \delta^{(4)}\left(p_{\chi}-\sum_{f} p_{f}\right) .
$$

By assuming a constant matrix element and massless final states, the phase space factor can be integrated in the rest frame of the decaying particle, yielding

$$
\mathrm{PS}_{n_{f}} \equiv \int\left(\prod_{f} \frac{d^{3} p_{f}}{2 \pi^{3}} \frac{1}{2 E_{f}}\right)(2 \pi)^{4} \delta^{(4)}\left(p_{\chi}-\sum_{f} p_{f}\right)=\frac{1}{2(4 \pi)^{2 n_{f}-3}} \frac{m_{\chi}^{2 n_{f}-4}}{\left(n_{f}-1\right) !\left(n_{f}-2\right) !}
$$

So, for example, the phase space factors up to $n_{f}=4$ are: $\mathrm{PS}_{2}=\frac{1}{8 \pi}, \mathrm{PS}_{3}=\frac{m_{\chi}^{2}}{256 \pi^{3}}$, $\mathrm{PS}_{4}=\frac{m_{\chi}^{4}}{24576 \pi^{5}}$.

In the case of a dimension $d$ effective operator, the amplitude squared for $n_{f}$ particles in the final state can be estimated by naive dimensional analysis (NDA) as

$$
\left|\mathcal{M}\left(m_{\chi} \rightarrow\left\{p_{f}\right\}\right)\right|_{\mathrm{NDA}}^{2}=\frac{\left(\frac{v}{\sqrt{2}}\right)^{2 n_{c}}}{\Lambda_{\mathrm{eff}}^{2(d-4)}} m_{\chi}^{2 d-2 n_{f}-2 n_{c}-6}
$$

where we also included the possibility of $n_{c}$ condensations of the Higgs boson. Hence, by putting eqs. (3.3)-(3.5) together, we get the following expression for the total width for $m_{\chi} \gg v$

$$
\Gamma_{\mathrm{NDA}}=\frac{1}{4(4 \pi)^{2 n_{f}-3}} \frac{m_{\chi}^{2 d-2 n_{c}-7}}{\left(n_{f}-1\right) !\left(n_{f}-2\right) !} \frac{\left(\frac{v}{\sqrt{2}}\right)^{2 n_{c}}}{\Lambda_{\mathrm{eff}}^{2(d-4)}} .
$$

Unless differently specified, we compute the lifetimes of the states decaying through the non-renormalizable operators in tables 1-2 using eq. (3.6). Whenever multiple operators can be responsible for the decay of $\chi$, we sum over the several widths assuming the operators contribute with the same Wilson coefficient. What is missing in eq. (3.6) with respect to the full decay width are the relevant $\mathrm{SU}(2)_{L}$ Clebsch-Gordan coefficients, symmetry, color and flavor factors, the kinematical dependence of the matrix element, the masses of the final states and finally mixing effects induced when scalar $\chi$ obtain VEVs. In the region $m_{\chi} \gg v$ all of these are expected to give $\mathcal{O}(1)$ corrections. When more accuracy is required, for example when setting BBN bounds, we take all these factors into account, computing the relevant decay widths explicitly. 


\begin{tabular}{|c|c|c|c|}
\hline Spin & $\chi$ & $Q_{\mathrm{LP}}$ & $\mathcal{O}_{\text {decay }}$ \\
\hline 0 & $(1,2,5 / 2)$ & 3 & $\chi^{\dagger} H e^{c} e^{c}$ \\
0 & $(1,5,1)$ & $-1,1,2,3$ & $\chi^{\dagger} H H H H^{\dagger}$ \\
0 & $(1,5,2)$ & $1,2,3,4$ & $\chi^{\dagger} H H H H$ \\
0 & $(\overline{3}, 2,11 / 6)$ & $7 / 3$ & $\chi H^{\dagger} u^{c} u^{c}+\chi^{\dagger} H d^{c} e^{c}$ \\
0 & $(3,3,5 / 3)$ & $8 / 3$ & $\chi^{\dagger} H q e^{c}+\chi H^{\dagger} u^{c} \ell$ \\
0 & $(3,4,1 / 6)$ & $5 / 3$ & $\chi H^{\dagger} q q+\chi^{\dagger} H q \ell$ \\
0 & $(\overline{3}, 4,5 / 6)$ & $7 / 3$ & $\chi^{\dagger} H q q+\chi H^{\dagger} q \ell$ \\
0 & $(\overline{6}, 2,7 / 6)$ & $5 / 3$ & $\chi^{\dagger} H d^{c} d^{c}$ \\
0 & $(8,3,1)$ & 2 & $\chi H^{\dagger} q u^{c}+\chi^{\dagger} H q d^{c}$ \\
\hline
\end{tabular}

Table 6. Extra states decaying through off-shell cascades.

\subsubsection{Cascade decays}

Whenever a Higgs doublet is contained in a SM-invariant operator, it can happen that not all the $\mathrm{SU}(2)_{L}$ components of the multiplet $\chi$ can directly decay through the effective operator. This is easily understood by going to the unitary gauge, where some of the $\mathrm{SU}(2)_{L}$ contractions end up into the goldstone directions of the Higgs doublet. See appendix B for a description of the $\mathrm{SU}(2)_{L}$ decompositions of the relevant operators. Depending on the mass spectrum, the cases where the LP cannot directly decay through the effective operator are displayed in table 6.

It is possible, however, for the LP to cascade decay via off-shell heavier components (which eventually decay through the effective operator) and $W$ bosons, thus resulting in lifetimes which are typically larger than in the case of the direct decay. Moreover, these decay rates must be evaluated numerically since the NDA formula in eq. (3.6) cannot be straightforwardly applied due to the strong momentum dependence of the matrix element.

For the computation of the decay width of the $(1,2,5 / 2)_{S}$ multiplet component with $Q=3, \chi_{+3}$, under the assumption that $\chi_{+3}$ is lighter than (or degenerate with) $\chi_{+2}$, we take into account the decays into two leptons and into two leptons together with a Higgs boson. The relevant Feynman diagrams are shown in figure 6. The numerical phase space integration is performed with the help of RAMBO [40] and we neglect the effects of lepton masses.

For the $(1,5,1)_{S}$ and $(1,5,2)_{S}$ multiplets, longer decay chains are possible for the multiple charged components of the multiplet. In addition, for the neutral states within these multiplets the effective operator induces a mixing with the Higgs boson which in turn generates direct couplings to SM vector bosons. These contributions to the lifetimes do not decouple for large $m_{\chi}$ and hence need to be taken into account over the whole considered mass range. For all multiplet components we thus consider decays with final states comprising of $2-4 \mathrm{SM}$ gauge or Higgs bosons. The numerical results have been obtained with Madgraph 5 [41] using FeynRules [42] generated model files. 

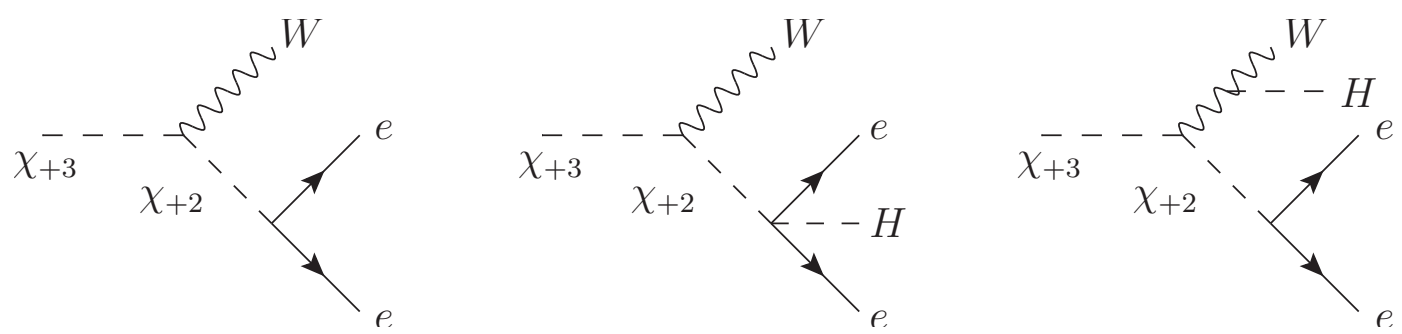

Figure 6. Feynman diagrams for the cascade decay of the $\chi_{+3}$ component of the $(1,2,5 / 2)_{S}$ state for $m_{\chi+3}<m_{\chi+2}$.

$(1,2,5 / 2)_{\mathrm{S}}, \mathrm{Q}=3$

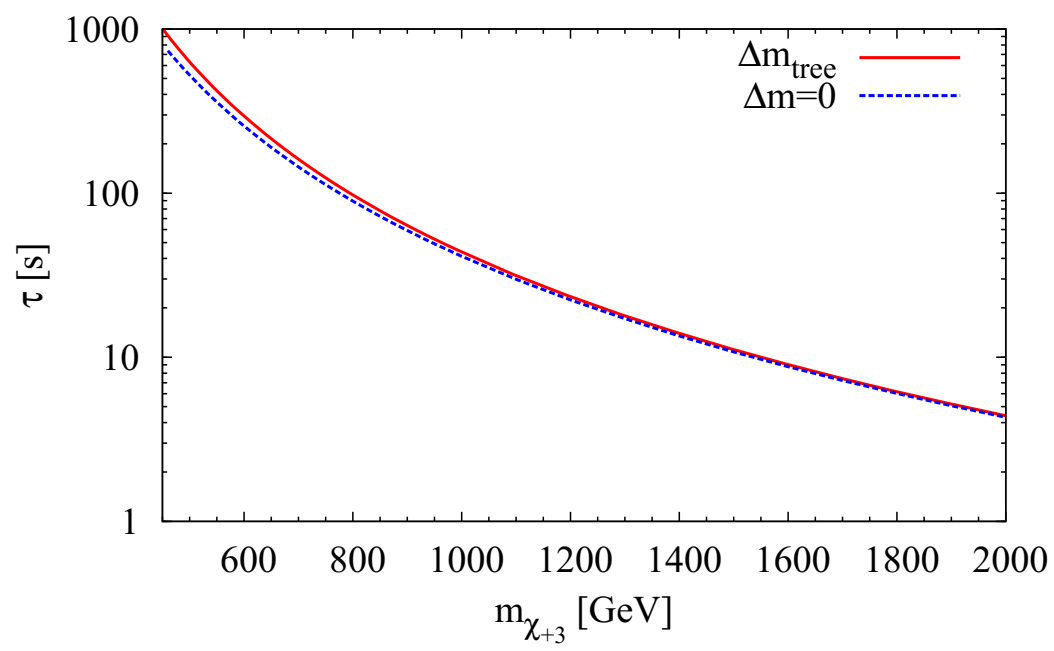

Figure 7. Lifetime as a function of the mass of the $Q=3$ component of the multiplet $(1,2,5 / 2)$, if it is the lightest. The red solid curve shows the lifetime for a NDA estimated tree-level mass splitting between $\chi_{+3}$ and $\chi_{+2}$ components, while the blue dashed curve represents the zero mass splitting limit.

Finally, the cascade decays of the colored cases can be estimated from the one of the $\chi_{+3}$ component of the $(1,2,5 / 2)_{S}$ multiplet by appropriate replacements of ClebschGordan coefficients and by multiplying with the respective color factors. Note that an accurate evaluation of the decay rates for the colored cases is not necessary since their relic abundance turns out to be very suppressed resulting in no relevant BBN constraints. More details can be found in section 4 .

The $\mathrm{SU}(2)_{L}$ factors needed in the evaluation of the cascade decays are exemplified in appendix B. For all the cases the cut-off scale $\Lambda_{\text {eff }}$ was set to $10^{15} \mathrm{GeV}$. We do not include off-shell effects of the $W$ bosons in the computation of the lifetimes. ${ }^{9}$

In figure 7 the lifetime of the $Q=3$ component of $(1,2,5 / 2)_{S}$ is shown as a function of its mass, assuming that it is lighter than the $Q=2$ component and hence decays via an off-shell $\chi_{+2}$. The blue dashed curve shows the lifetime in the zero mass splitting

\footnotetext{
${ }^{9}$ In ref. [43] it was shown that in the case of stop decays these off-shell effects can be numerically relevant for mass differences between the decaying particle and the decay products up to $35 \mathrm{GeV}$.
} 
$(1,5,1)_{\mathrm{S}}$

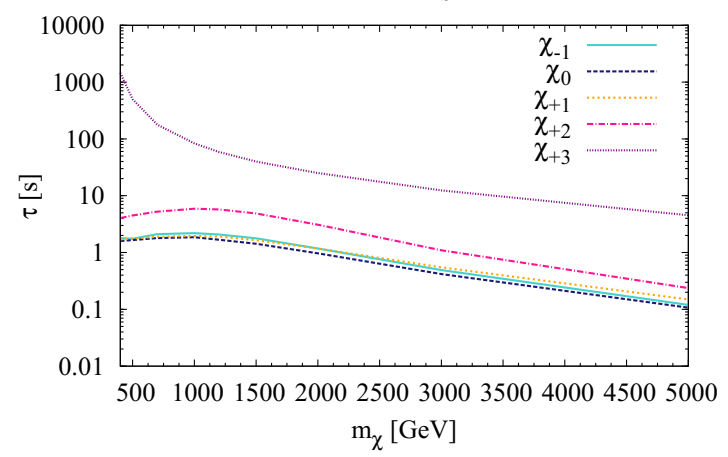

$(1,5,2)_{\mathrm{S}}$

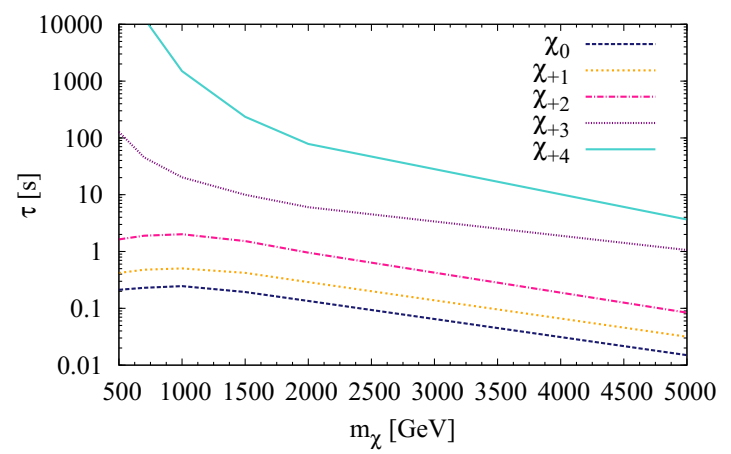

Figure 8. Left: lifetimes of the $Q=0$ (dark blue dashed), $Q=1$ (yellow large dots), $Q=-1$ (turquoise solid), $Q=2$ (pink dash-dotted) and $Q=3$ (violet small dots) states of the $(1,5,1)_{S}$ multiplet, assuming for each curve that the respective component is the lightest one. Right: same as for the left-hand side but for $(1,5,2)_{S}$. The turquoise solid line corresponds to the $Q=4$ lightest state.

approximation, while the red solid one stands for a NDA estimated tree-level mass splitting as given in eq. (2.15). In the plot we assume that the $d=5$ operator involves only one lepton flavor. If $\chi$ couples in the same way to all three flavors the corresponding lifetimes are reduced by a factor of three. From figure 7 it can be inferred that the presence of tree-level mass splitting only affects the lifetimes for low masses of $\chi_{+3}$, of the order $\mathcal{O}(v)$. For larger masses it quickly becomes completely irrelevant and we henceforth work in the zero mass splitting limit whenever computing cascade decays.

In figure 8 , we show the lifetimes of all the components of the $(1,5,1)_{S}$ (left panel) and $(1,5,2)_{S}$ (right panel) multiplet assuming inter-multiplet mass degeneracy. As it can be inferred from the plot, the same scaling behavior of all the components for large $m_{\chi}$ is found as expected in the $\mathrm{SU}(2)_{L}$ limit. The lifetimes of $\chi_{+2}, \chi_{+3}$ (and $\chi_{+4}$ in case of $\left.(1,5,2)_{S}\right)$ are larger due to the fact that a smaller number of final states is available, especially at lower masses, and hence the decay widths are suppressed. For such long lifetimes there are potential issues with cosmology (see section 4.2).

\subsubsection{Loop-induced decays}

In all the SM extensions considered in tables 1-2 there is always an operator responsible for the decay of the new multiplet that is linear in $\chi$, except in the case of the (real) scalar multiplet with SM gauge quantum numbers $(1,7,0)$. In this case the operator responsible for $\chi$ decay is $\chi \chi \chi H^{\dagger} H .{ }^{10}$ This can be understood by simply noticing that the SM extended with a real $(1,7,0)$ scalar has an accidental $Z_{2}$ symmetry, $\chi \rightarrow-\chi$, at the renormalizable level and the presence of an operator trilinear in $\chi$ clearly breaks such a symmetry. We note that in the context of minimal DM [4] this $d=5$ operator and its effect on the scalar septuplet lifetime have been previously overlooked. The decay only proceeds at one-loop

\footnotetext{
${ }^{10}$ Different $\mathrm{SU}(2)_{L}$ contractions give rise to different independent operators. In this section we consider the case where two fields $\chi$ are contracted in a $j=4$ weak isospin multiplet.
} 

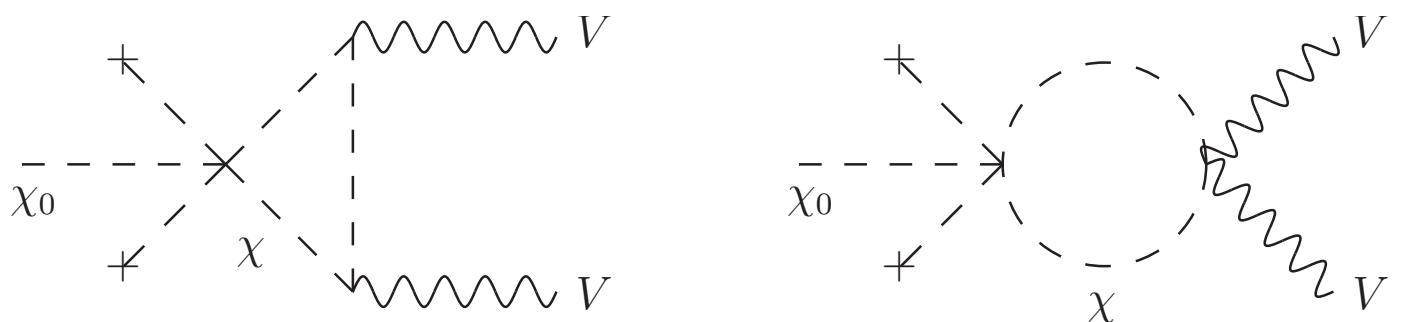

Figure 9. Feynman diagrams for the loop decay of the $\chi_{0}$ component of the $(1,7,0)_{S}$ multiplet with $V V=\gamma \gamma, \gamma Z, Z Z, W^{+} W^{-}$. Electroweak VEV insertions are denoted by a cross.

level and, depending on the nature of the lightest particle in the multiplet, can result in the following final states with EW gauge bosons ${ }^{11}$

- $\chi_{0}$ : the possible two-body final states are $\gamma \gamma, \gamma Z, Z Z$ and $W^{+} W^{-}$. The relevant Feynman diagrams are shown in figure 9. By neglecting the gauge boson masses in the final state we get

$$
\Gamma_{\chi_{0}}=\frac{857 C_{0}^{2}}{441548 \pi^{5}} \frac{g^{4} v^{4}}{\Lambda_{\mathrm{eff}}^{2} m_{\chi}}=5.9 \times 10^{-8} \mathrm{~s}^{-1}\left(\frac{10^{15} \mathrm{GeV}}{\Lambda_{\mathrm{eff}}}\right)^{2}\left(\frac{1 \mathrm{TeV}}{m_{\chi}}\right),
$$

where $C_{0} \approx-0.0966$ is a numerical factor coming from the evaluation of the relevant Passarino-Veltman functions. We observe that even for an EFT cut-off at the Planck scale, the fast decay of the neutral component of the septuplet effectively rules out this particular minimal scalar DM candidate [44].

- $\chi_{+1}$ : the two-body final states are $W \gamma$ or $W Z$, with a decay rate given by

$$
\Gamma_{\chi+1}=\frac{9 C_{0}^{2}}{34496 \pi^{5}} \frac{g^{4} v^{4}}{\Lambda_{\mathrm{eff}}^{2} m_{\chi}}=7.9 \times 10^{-9} \mathrm{~s}^{-1}\left(\frac{10^{15} \mathrm{GeV}}{\Lambda_{\mathrm{eff}}}\right)^{2}\left(\frac{1 \mathrm{TeV}}{m_{\chi}}\right) .
$$

- $\chi_{+2}$ : there is only a two-body decay into $W W$, yielding

$$
\Gamma_{\chi+2}=\frac{9245 C_{0}^{2}}{2207744 \pi^{5}} \frac{g^{4} v^{4}}{\Lambda_{\text {eff }}^{2} m_{\chi}}=1.3 \times 10^{-7} \mathrm{~s}^{-1}\left(\frac{10^{15} \mathrm{GeV}}{\Lambda_{\mathrm{eff}}}\right)^{2}\left(\frac{1 \mathrm{TeV}}{m_{\chi}}\right) .
$$

- $\chi_{+3}$ : in this case there are no two-body decay channels into gauge bosons, while it is possible to show that if we ignore the effect of SM fermions $\chi_{+3}$ cannot decay into an odd number of gauge bosons. ${ }^{12}$ Hence, we do expect that the leading contribution to this decay will be given by a final state containing four gauge bosons. Though we did not explicitly compute this decay rate, we can quote (and use in the numerical analysis) an NDA estimate given by

$$
\Gamma_{\chi+3}=\frac{1}{3145728 \pi^{7}} \frac{g^{8} v^{4}}{\Lambda_{\mathrm{eff}}^{2} m_{\chi}}=1.9 \times 10^{-11} \mathrm{~s}^{-1}\left(\frac{10^{15} \mathrm{GeV}}{\Lambda_{\mathrm{eff}}}\right)^{2}\left(\frac{1 \mathrm{TeV}}{m_{\chi}}\right) .
$$

\footnotetext{
${ }^{11}$ For very large $\chi$ masses, final states containing Higgs bosons might be important as well.

${ }^{12}$ At the one-loop level there are no contributions from SM fermions and the charge conjugation $C$ transformation is a symmetry of the gauge and scalar sectors. The selection rules for these decay channels follow from the presence of this symmetry.
} 
We end this section by noting that for $\Lambda_{\text {eff }} \gtrsim 10^{15} \mathrm{GeV}$ the loop-induced lifetimes when combined with cosmological considerations preclude the scalar septuplet to be within the kinematical reach of the LHC (see section 4.2 for details).

\section{Cosmology}

Most of the accidentally safe SM extensions are characterized by color- and weak multiplets of scalars or fermions, with weak-scale masses and no renormalizable interactions beyond their couplings to the SM gauge bosons (and the Higgs portal operators in the case of scalars). Thus they will be produced and thermalized in the early Universe, eventually freezing-out once their thermalizing interactions become slower than the Hubble expansion rate $H(T)$. The details depend somewhat on the mass hierarchy within the $\chi$ multiplets but the decay rates of the lightest $\chi_{i}$ components (through higher dimensional operators) are typically much smaller than $H(T)$ at freeze-out for both weakly and strongly interacting $\chi$. We thus have effectively a two step process and we can treat freeze-out and decay separately.

In case $\chi$ is a color singlet, the cosmological relic abundance will generically be determined by its (co)annihilations into EW gauge bosons resulting in a cosmological density of $\Omega_{\chi} h^{2} \sim 0.01$. On the other hand, the final relic abundance of a colored multiplet is determined in two stages. At temperatures $T \sim m_{\chi} / 30$ the relic abundance is determined by perturbative QCD annihilations resulting in $\Omega_{\chi} h^{2} \sim 10^{-3}$. Then, $\chi$ undergoes a second stage of annihilation after the QCD phase transition, further reducing its relic abundance to a value roughly three orders of magnitude smaller [45].

The $\chi$ lifetimes determine at which cosmological epoch they will decay. Such decays will involve the creation of energetic SM particles, which can produce a variety of observable effects. First, the decays of heavier multiplet components into the lightest $\chi_{i}$ state always happen well before nucleo-synthesis and give a negligible entropy release. On the other hand, if the lightest $\chi_{i}$ states can decay through $d=5$ operators, their lifetimes are at least of the order $\left(0.1-10^{5}\right) \mathrm{s}$, and may thus affect the primordial generation of light nuclear elements [46]. For longer lifetimes of the order $\left(10^{12}-10^{13}\right) \mathrm{s}, \chi_{i}$ decays would create distortions in the thermalization of the cosmic microwave background (CMB) before recombination. Such distortions of the spectrum by the injection of high-energy photons into the plasma lead to strong constraints [47]. Decays of $\chi_{i}$ after recombination can give rise to photons that free-stream to us, and are visible in the diffuse gamma ray background [48]. Observations by Fermi LAT [49] limit the flux of these gamma rays and thus constrain such scenarios. In general these observations of the diffuse gamma ray background rule out $\chi_{i}$ with lifetimes between $\left(10^{13}-10^{26}\right)$ s. If $\chi_{i}$ only decay through $d \geq 6$ operators, they will survive to the present day. In case they are integer charged, they would act as heavy positively charged nucleons, producing anomalously heavy isotopes. A combination of measurements places severe limits on the abundance of terrestrial heavy elements today [50], effectively excluding such scenarios. ${ }^{13}$ Alternatively, if their charge is a non-integer fraction of that of the electron, they are excluded by the null results of searches for fractionally charged particles in bulk matter on Earth or meteoritic material [52, 53].

\footnotetext{
${ }^{13}$ We note however that in principle these bounds can be evaded for $m_{\chi} \gg \mathrm{TeV}$ [51].
} 
Finally, sufficiently stable neutral $\chi_{i}$ can form (a fraction of) dark matter, a possibility, which has been thoroughly covered in the literature [4-7]. In principle, one could think about introducing an infinitesimal hypercharge $\left(\epsilon_{Y}\right)$ which would make $\chi$ absolutely stable but still pass all the cosmological bounds. This would open up additional DM candidate scenarios like the complex scalars $\left(1,1, \epsilon_{Y}\right),\left(1,3, \epsilon_{Y}\right),\left(1,5, \epsilon_{Y}\right)$ or the Dirac fermions $\left(1,1, \epsilon_{Y}\right),\left(1,3, \epsilon_{Y}\right)$. Representations having $Y \neq 0$ for $\epsilon_{Y} \rightarrow 0$ are excluded by direct DM searches [4]. On the other hand, higher-dimensional $\mathrm{SU}(2)_{L}$ representations have a Landau pole below $10^{15} \mathrm{GeV}$ (cf. table 9 ). We will not entertain such a possibility any further since it is a rather simple distortion of the minimal DM setup (see for instance [7]). We also refer the reader to existing literature for more details on experimental bounds on $\epsilon_{Y}$ (e.g. [54]).

We close this section with a few general comments about the possible interpretation of cosmological DM within our framework. First of all, we note that the microscopic nature of the DM is still uncertain. For example Massive Astrophysical Compact Halo Object (MACHO) made of ordinary baryons (like black holes or neutron stars) could in principle be a viable option. It is known, however, that in such cases departures from the standard Big Bang theory are needed. The present cosmological data and various theoretical considerations favor the hypothesis of particle DM. Besides the minimal DM cases, requiring DM of this type in our framework means departing from minimality. The easiest possibility then is to assume, on top of the (non DM) accidental matter state, the presence of the fermionic minimal DM multiplet at $\sim 10 \mathrm{TeV}$. However, this works only for some accidental matter states. In other cases extra $d=5$ operators can trigger too fast decay of the minimal DM candidate. Additional possibilities include the presence of extra gauge interactions where the stability of DM is again guaranteed by an accidental symmetry of the new gauge sector (for a recent work along these lines see [55]) or axion DM with PQ symmetry breaking above $\Lambda_{\text {eff }}[56-58]$.

\subsection{Relic abundance}

We first consider scenarios with uncolored $\chi$, where its lightest component is electrically charged. In these cases, direct searches already limit $m_{\chi} \gg m_{Z}$ (see section 5.2) and we can compute the relevant thermally-averaged cross-sections in the $\mathrm{SU}(2)_{L}$-symmetric limit. This approach is valid as long as all $\mathrm{SU}(2)_{L}$ multiplet components are present in the thermal plasma.

For inter-multiplet splittings of typical radiative size all heavier $\chi_{i}$ components decay into the lightest one with lifetimes (cf. figure 5) which can be comparable or even shorter than the inverse Hubble rate at freeze-out (typically $\mathcal{O}\left(10^{-11} \mathrm{~s}\right)$ ). Thus the abundance of the lightest $\chi_{i}$ component (before itself starts decaying) is actually described by the sum of the densities of all $\chi_{i}$ states. And as long as $\chi_{i} \leftrightarrow \chi_{j}$ conversion rates are in equilibrium at freeze-out (which is always the case for color singlet weakly interacting $\chi$ ), the actual $\chi_{i} \rightarrow \chi_{j} X$ rates do not affect the total relic abundance [59], and the $\mathrm{SU}(2)_{L}$ symmetric approximation can be justified.

Finally, we also ignore thermal corrections. They mainly induce thermal mass splittings of the order $\Delta m_{\chi} \sim\left(g^{2} T\right)^{2} / m_{\chi}$, which can be neglected at the level of precision we are considering here [5]. 
Due to the above approximations we can write a single Boltzmann equation that describes the evolution of the total abundance of all components $\chi_{i}$ of the multiplet as a whole. In particular, it includes all co-annihilations in the form of $\sum_{i j} \sigma_{A}\left(\chi_{i} \chi_{j} \rightarrow \mathrm{SM}\right.$ particles). The final $\chi$ abundance can be well approximated as [4]

$$
Y_{\chi} \equiv \frac{n_{\chi}(T)}{s(T)} \approx \sqrt{\frac{180}{\pi g_{\mathrm{SM}}}} \frac{1}{m_{\mathrm{Pl}} T_{f}\langle\sigma v\rangle}, \quad \frac{m_{\chi}}{T_{f}} \approx \ln \frac{g_{\chi} m_{\chi} m_{\mathrm{Pl}}\langle\sigma v\rangle}{240 \sqrt{g}_{\mathrm{SM}}}
$$

where $g_{\chi}$ is the number of degrees of freedom of a whole $\chi$ multiplet including anti-particles in case of complex representations, $g_{\mathrm{SM}}$ is the number of SM degrees-of-freedom in thermal equilibrium at the freeze-out temperature $T_{f}$ (cf. [60]), and $s$ is their total entropy. The typical freeze-out temperature is $T_{f} \sim m_{\chi} / 26 \ll m_{\chi}$, such that we can keep only the dominant s-wave (co)annihilation processes. The relevant formulae for the corresponding thermally averaged annihilation cross-sections $\langle\sigma v\rangle$ into $\mathrm{SU}(2)_{L} \otimes \mathrm{U}(1)_{Y}$ vector bosons for both scalar and fermionic $\chi$ with generic $\mathrm{SU}(2)_{L} \otimes \mathrm{U}(1)_{Y}$ quantum numbers can be found in [4]. The resulting $Y_{\chi}$ estimates are within $10 \%$ of the more complete treatment including $p$-wave annihilations and renormalization of the SM gauge couplings [5]. However, for $m_{\chi} \gtrsim 1 \mathrm{TeV}$, the relic abundance is expected to be further reduced by $\mathcal{O}(1)$ nonperturbative (Sommerfeld) corrections due to the electrostatic Coulomb force effects [61]. In case of scalars, additional renormalizable Higgs portal interactions can also contribute to the annihilation cross section deferring freeze-out. In light of this our estimates of $Y_{\chi}$ using eq. (4.1) with dominant EW gauge boson contributions to $\langle\sigma v\rangle$ can be considered as upper bounds on the actual relic abundances of $\chi$.

In the case of colored $\chi$, one needs to consider two separate regimes of annihilation. The first era is before the QCD phase transition when all $\chi$ components are freely propagating in the QCD plasma and the annihilation cross-section can be determined using perturbative QCD. The second era is after the QCD phase transition when the heavier multiplet components have decayed to the lightest $\chi$, which in turn have become confined in color neutral bound states. The annihilation cross section in this second period turns out to be much higher than in the first, thus leading to a second period of annihilation which completely determines the final $\chi$ relic abundance [45]. In particular, heavy colored particles are confined within hadronic states of typical size $R_{\text {had }} \sim \mathrm{GeV}^{-1}$ which annihilate with a geometrical cross section yielding $\langle\sigma v\rangle \sim \pi R_{\text {had }}^{2} \sqrt{T_{B} / m_{\chi}}$, where $T_{B} \sim 180 \mathrm{MeV}$ is the temperature at which QCD confines and hadronic bound states form. The final $\chi$ relic abundance can thus be approximated as

$$
Y_{\chi} \sim 10^{-17}\left(\frac{R_{\mathrm{had}}}{\mathrm{GeV}^{-1}}\right)^{-2}\left(\frac{T_{B}}{180 \mathrm{MeV}}\right)^{-2 / 3}\left(\frac{m_{\chi}}{\mathrm{TeV}}\right)^{1 / 2}
$$

where we have used eq. (4.1) with $T_{f}=T_{B}$ and $g_{S M} \sim 15$ just below the QCD phase transition. The annihilation proceeds through intermediate excited bound states which decay by radiating away photons before annihilating into quarks and gluons [45]. These processes need to be considered carefully, since such late decays to photons and hadronic jets could affect nucleosynthesis [62]. In case of electrically charged $\chi_{i}$, this process is fast 


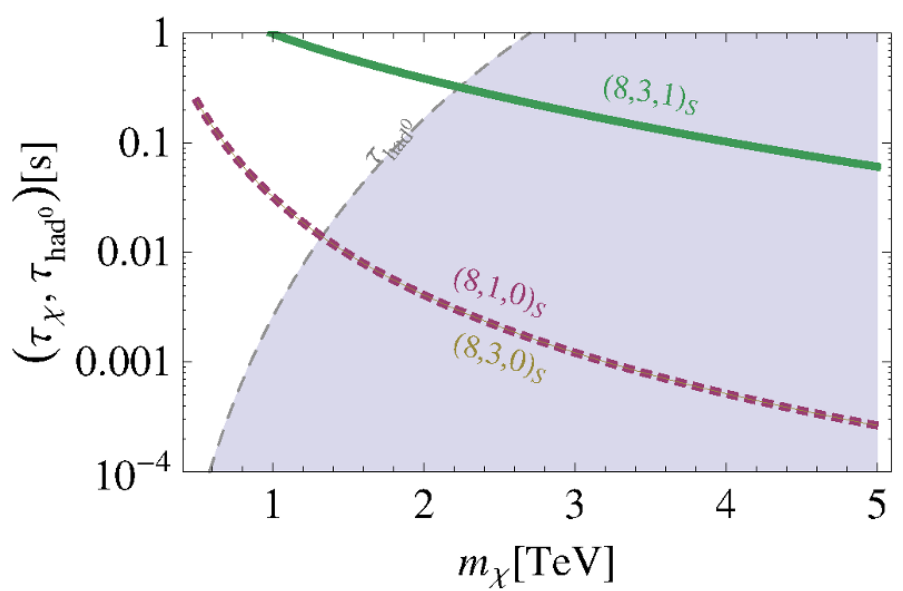

Figure 10. Comparison of NDA estimates for the $\chi_{0}$ lifetimes $\left(\tau_{\chi}\right)$ in case of colored multiplets $(8,1,0)_{S}$ (drawn in thick dashed purple), $(8,3,0)_{S}$ (drawn in thin brown) and $(8,3,1)_{S}$ (drawn in thick green) with the annihilation lifetimes of the corresponding $\chi_{0}$ hadronic bound states $\left(\tau_{\text {had }^{0}}\right.$, drawn in thin dashed gray). In the shaded region, the $\chi_{0}$ decay before their hadronic bound states fully annihilate. See text for details.

with a lifetime of

$$
\tau_{\mathrm{had}^{+}} \sim \frac{\left[\alpha_{s}\left(m_{\chi}\right)\right]^{1 / 2} m_{\chi}^{2}}{\alpha_{\mathrm{EM}} \Lambda_{\text {had }}^{3}} \sim 3 \times 10^{-17} \mathrm{~s}\left(\frac{\alpha_{s}\left(m_{\chi}\right)}{0.1}\right)^{1 / 2}\left(\frac{\Lambda_{\mathrm{had}}}{\mathrm{GeV}}\right)^{-3}\left(\frac{m_{\chi}}{\mathrm{TeV}}\right)^{2} .
$$

where $\Lambda_{\text {had }} \sim 1 \mathrm{GeV}$ is related to the QCD string tension $\sigma$ via $\sigma \sim \Lambda_{\text {had }}^{2}$. On the other hand, for electrically neutral $\chi_{i}$, radiation of photons is loop suppressed, leading to a much longer annihilation process

$$
\tau_{\text {had }^{0}} \lesssim \frac{4 \pi m_{\chi}^{6}}{\alpha_{\mathrm{EM}}^{2} \Lambda_{\text {had }}^{7}}\left(\frac{T_{B}}{\Lambda_{\text {had }}}\right)^{7 / 3} \sim 1 \mathrm{~s}\left(\frac{m_{\chi}}{2.7 \mathrm{TeV}}\right)^{6}\left(\frac{\Lambda_{\text {had }}}{\mathrm{GeV}}\right)^{-28 / 3}\left(\frac{T_{B}}{180 \mathrm{MeV}}\right)^{7 / 3}
$$

where the inequality is due to neglected non-local contributions to the decay rate. This scenario however, only applies to our cases $\chi \sim(8,1,0)_{S},(8,3,0)_{S}$ and $(8,3,1)_{S}$ when the lightest component is neutral. In figure 10 we plot the relevant lifetimes $\tau_{\chi}$ and $\tau_{\text {had }^{0}}$ as a function of $\chi$ mass. We observe that in the low mass regime, $\tau_{\mathrm{had}^{0}}<\tau_{\chi}$ and we can use the non-perturbative result in eq. (4.2) to estimate the final $\chi$ abundance. However in the heavy $\chi$ limit, $\tau_{\text {had }^{0}}>\tau_{\chi}$ and so $\chi$ decays before the second stage annihilation process is completed. In this case the relevant abundancies are those after the first stage of annihilation above the QCD phase transition. We can estimate them using the general $\mathrm{SU}(N)$ annihilation cross-sections computed in [63] after exchanging the relevant $\mathrm{SU}(N)$ group invariants and correcting for the different number of degrees of freedom. In particular for the adjoint representation of QCD $\sum_{i, j, a, b}\left|\left\{T^{a}, T^{b}\right\}_{j i}\right|^{2}=216$ and $\sum_{i, j, a, b}\left|\left[T^{a}, T^{b}\right]_{j i}\right|^{2}=$ 72. Velocity expanding the resulting $\chi \chi \rightarrow g g$ cross section we obtain (cf. [64]),

$$
\langle\sigma v\rangle=\frac{27 \pi \alpha_{s}^{2}}{g_{\chi} m^{2}}
$$

in the conventions of [4]. In estimating the resulting relic abundance we can safely assume that weak interactions keep $\chi_{i} \leftrightarrow \chi_{j}$ processes in equilibrium until decoupling [59]. We 
have also checked that employing eqs. (4.1) and (4.5) and using the running $\alpha_{s}\left(2 m_{\chi}\right)$ to estimate the relic abundance reproduces the results of the full leading order perturbative QCD calculation and integration of the Boltzmann equation to within 20\% (in agreement with similar results for the fundamental QCD representation in [63]). Non-perturbative Sommerfeld corrections are expected to lead to an $\mathcal{O}(1)$ reduction in the final result and so our estimates can again be taken as upper bounds on the actual relic abundance of color octet scalar $\chi$ above the QCD phase transition. In the intermediate $m_{\chi}$ regime when $\tau_{\text {had }^{0}} \sim \tau_{\chi}$ the actual evolution of the $\chi$ number density in the primordial plasma depends on the detailed dynamics of the neutral $\chi$ hadron annihilation and decays, the evaluation of which is beyond the scope of our study. As we show in the next section however, these details are never relevant, since they do not lead to observational constraints.

\subsection{Implications for Big Bang nucleosynthesis}

In general, nucleosynthesis of primordial elements in the early Universe represents a sensitive probe of any metastable relic with lifetime of about $1 \mathrm{~s}$ or longer [46,65]. The constraints come from two classes of processes: injection of very energetic photons or hadrons from decays during or after BBN adds an additional non-thermal component to the plasma and can modify the abundances of the light elements [66-70]; in addition, if the relic particle is electromagnetically charged, bound states with nuclei may arise that strongly enhance some of the nuclear rates and allow for catalysed production of e.g. ${ }^{6} \mathrm{Li}$, ${ }^{7} \mathrm{Li}[71-73]$. The Standard BBN prediction for the ${ }^{6} \mathrm{Li}$ abundance is actually significantly smaller than the observed one, so that the presence of a charged relic with appropriate lifetime can help reconciling $\mathrm{BBN}$ with the measured abundances of ${ }^{6} \mathrm{Li}$ and ${ }^{7} \mathrm{Li}[74-79]$.

In general, the decay can produce very energetic SM particles that can initiate either hadronic or electromagnetic showers in the plasma. The most stringent bounds are obtained for a relic that produces mostly hadronic showers, since electromagnetic particles like photons or electrons can thermalize very quickly by interacting with the tail of the CMB distribution until times of about $10^{6} \mathrm{~s}$. In the following we will consider the constraints for relics producing a small number of energetic hadronic jets with a branching ratio $\mathcal{B}_{\text {had }}=1$ and $E_{\text {had }} \sim m_{\chi}$, where $E_{\text {had }}$ is the decay energy released in the form of hadronic showers to obtain conservative upper bounds on $\chi$ number densities. This assumption is mostly valid if $\chi$ is colored (in particular in this case always $\mathcal{B}_{\text {had }}=1$ and $E_{\text {had }} \gtrsim m_{\chi} / 2$ ), while $\mathcal{B}_{\text {had }}<1$ is expected for non-colored $\chi$. Then the hadronic BBN bounds are relaxed accordingly by a factor $1 / \mathcal{B}_{\text {had }}$. Finally, for lifetimes $\tau_{\chi} \gtrsim 10^{4} \mathrm{~s}$, electromagnetic interactions start having a significant effect and the bounds above $\tau_{\chi} \gtrsim 10^{7} \mathrm{~s}$ become effectively independent of $\mathcal{B}_{\text {had }}$.

In practice there are three regions of lifetimes as discussed in [62]: for lifetimes $0.1 \mathrm{~s} \lesssim \tau_{\chi} \lesssim 100 \mathrm{~s}$ the dominant effect is the interconversion between protons and neutrons, that changes the ${ }^{4} \mathrm{He}$ abundance by overproducing it; at longer lifetimes $100 \mathrm{~s} \lesssim \tau_{\chi} \lesssim 10^{7} \mathrm{~s}$ hadrodissociation is the most efficient process and the bounds come from the non-thermal production of lithium and deuterium; finally at late times $10^{7} \mathrm{~s} \lesssim \tau_{\chi} \lesssim 10^{12}$ s photodissociation caused both by direct electromagnetic showers and by those generated by the daughter hadrons starts to dominate and result mainly in the overproduction of ${ }^{3} \mathrm{He}$. 
In the following we use the results from the general analysis of [62] for the second and the third lifetime regions. In particular the bound coming from the abundance of ${ }^{3} \mathrm{He}$ scales as $1 / m_{\chi}$ and does not depend on $\mathcal{B}_{\text {had }}$ (we neglect the decay energy released into neutrinos, which is always expected to be a small fraction of $m_{\chi}$ ). On the other hand due to the Li anomaly, in the second region we only consider bounds on $Y_{\chi}$ coming from the deuterium to hydrogen abundance ratio $(\mathrm{D} / \mathrm{H})$, which scale roughly as $E_{\text {had }}^{-1 / 2}$. Finally, we note that a charged thermal relic with $\tau_{\chi} \sim 10^{2}-10^{3}$ s and abundance just below the $\mathrm{D} / \mathrm{H}$ bound may (partly) ameliorate the standard BBN Lithium problems (cf. [80]).

The value of the observed ${ }^{4} \mathrm{He}$ abundance $Y_{p} \equiv 4\left(n_{\mathrm{He}} / n_{\mathrm{H}}\right) /\left(1+4 n_{\mathrm{He}} / n_{\mathrm{H}}\right) \approx$ $2\left(n_{n} / n_{p}\right) /\left(1+n_{n} / n_{p}\right)$ which dominates the constraints in the first lifetime region has been updated since the analysis of [62] and currently reads $Y_{p}=0.250(3)$ [65] to be compared with the prediction of standard $\mathrm{BBN}$ of $Y_{p}^{\mathrm{SBBN}}=0.2483(5)$ [81]. The bounds from [62] which assumed significantly smaller $Y_{p}$ thus need to be re-evaluated. For this purpose we numerically solve the relevant Boltzmann equations (cf. [60])

$$
\begin{aligned}
& -H(T) T \frac{d y_{\chi}}{d T}=-\frac{y_{\chi}}{\tau_{\chi}}, \\
& -H(T) T \frac{d y_{n}}{d T}=-\lambda_{n p} y_{n}+\lambda_{p n} y_{p}-\frac{y_{n}}{\tau_{n}}-\frac{\mathcal{B}_{\mathrm{had}}}{\tau_{\chi}} y_{\chi} K_{\chi}, \\
& -H(T) T \frac{d y_{p}}{d T}=-\lambda_{p n} y_{p}+\lambda_{n p} y_{n}+\frac{y_{n}}{\tau_{n}}+\frac{\mathcal{B}_{\mathrm{had}}}{\tau_{\chi}} y_{\chi} K_{\chi},
\end{aligned}
$$

where $y_{i} \equiv n_{i} / n_{b}$ and $n_{b}$ is the baryon number density (we use $\eta \equiv n_{b} / n_{\gamma}=6.1 \times$ $\left.10^{-10}\right), H(T)=\pi\left(T^{2} / m_{\mathrm{Pl}}\right) \sqrt{g_{\mathrm{SM}} / 90}$ is the Hubble rate and $\tau_{n}=880(1) \mathrm{s}$ is the neutron lifetime. We have furthermore defined $K_{\chi} \equiv K_{n \rightarrow p}-K_{p \rightarrow n}$ and $\lambda_{n p} \simeq \lambda_{p n} \exp (1 / y) \simeq$ $\left(1443 / \tau_{n}\right) y^{3}(y+0.25)^{2}[82,83]$, where $y=T / Q$ and $Q=1.293 \mathrm{MeV}$ is the neutron-proton mass difference. We have checked that this approximate form of $\lambda_{n p}$ and $\lambda_{p n}$ reproduces the final results using exact numerical integration of the weak nucleon conversion rates (cf. $[82,84]$ ) to better than $0.5 \%$. Finally, for the catalyzed nuclear conversion rates we employ the formulae for $K_{N \rightarrow N^{\prime}}$ including all the numerical inputs as defined in [62]. At temperatures much bigger than $Q$ we expect $y_{n}=\left(1-y_{p}\right)=1 /(1+\exp (Q / T))$ and $y_{\chi}$ given by its thermal relic abundance $\bar{y}_{\chi}$. Finally, the resulting ${ }^{4} \mathrm{He}$ abundance is well determined by $y_{n, p}$ at $\left(T \simeq 8.5 \times 10^{9} \mathrm{~K}\right)$ when BBN begins [81, 85]. As a cross-check of our approach, we have determined $Y_{p}$ in absence of $y_{\chi}$ and obtained $Y_{p}\left(\bar{y}_{\chi}=0\right)=0.243$, which is consistent with the expected precision in light of our approximations. In particular, neglected higher order effects would increase $Y_{p}$ by $2 \%$ [82], reproducing the standard BBN result. In setting constraints we use our estimates only to compute the deviations of $Y_{p}$ from the standard BBN value $\Delta Y_{p}=Y_{p}\left(\bar{y}_{\chi}\right)-Y_{p}\left(\bar{y}_{\chi}=0\right)$ and compare $Y_{p}^{\mathrm{SBBN}}+\Delta Y_{p}$ to the $2 \sigma$ region of the observed $Y_{p}$ value. As discussed above, the effects of $\chi$ decays on $Y_{p}$ scale as $1 / \mathcal{B}_{\text {had }}$, and for $E_{\text {had }} \gtrsim 100 \mathrm{GeV}$ (in the form of a fixed number of hadronic jets) also approximately as $E_{\text {had }}^{-1 / 3}$.

We finally determine the upper bound on the possible contributions of $\chi$ decays to $Y_{p}$ by fixing $\mathcal{B}_{\text {had }}=1$ and assuming $\chi$ decay to two hadronic jets. The resulting effects then scale as $m_{\chi}^{-1 / 3}$ and we can use a single reference value to constrain the abundances of $\chi$ 

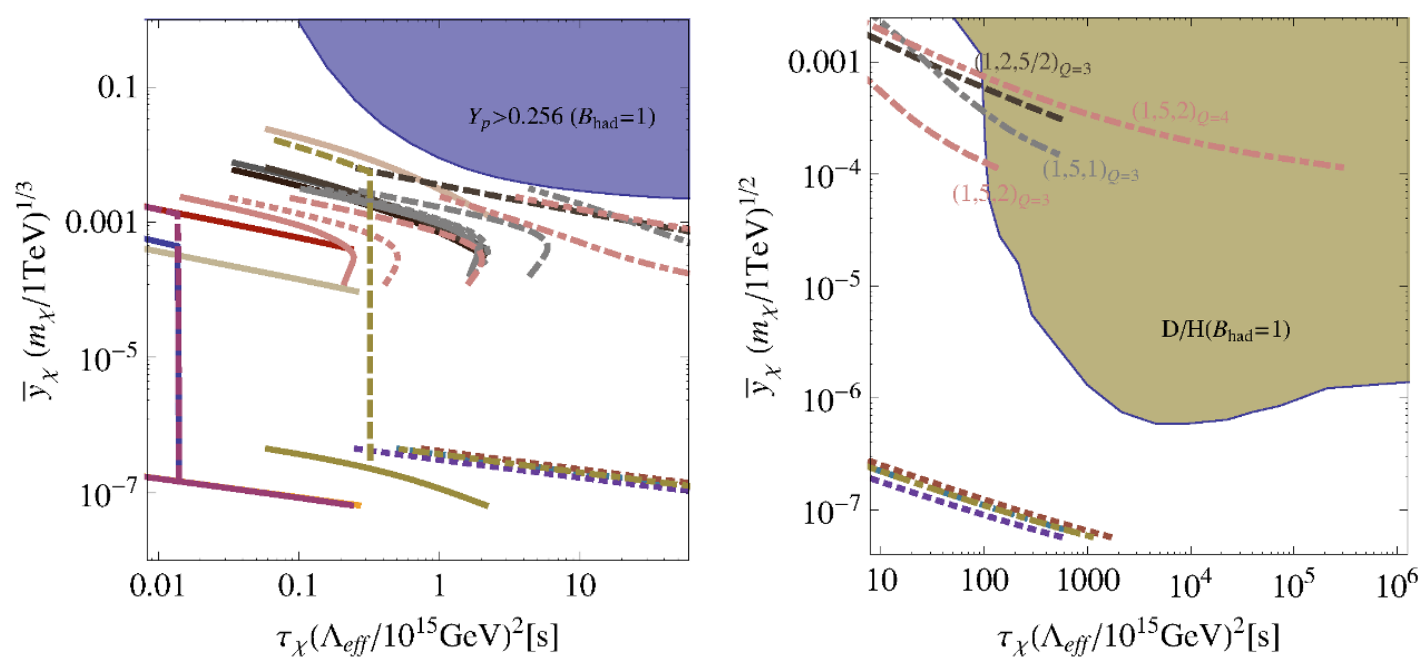

Figure 11. Comparison of BBN $Y_{p}$ (left hand panel) and D/H (right hand panel) constraints on the abundances and lifetimes of metastable hadronically decaying particles with the corresponding estimates for the cases of viable $\chi$ multiplets. Each line, going from bottom to top, corresponds to the mass range $0.5 \mathrm{TeV}<m_{\chi}<5 \mathrm{TeV}$. The only explicitly labeled examples are $(1,5,2)_{S, Q_{\mathrm{LP}}=3}$, $(1,5,2)_{S, Q_{\mathrm{LP}}=4},(1,5,1)_{S, Q_{\mathrm{LP}}=3}$ and $(1,2,5 / 2)_{S, Q_{\mathrm{LP}}=3}$, which are potentially constrained. The case $(1,7,0)$ is not shown as its lifetime is longer than $10^{6} \mathrm{~s}$ for $0.5 \mathrm{TeV}<m_{\chi}<5 \mathrm{TeV}$ and $\Lambda_{\text {eff }}=$ $10^{15} \mathrm{GeV}$.

at different $m_{\chi}$. As discussed above, this leads to a conservative $\mathcal{O}(1)$ overestimate of the actual $Y_{p}$ constraints for decays of $\chi$ involving also uncolored final states. The comparison of the lifetimes and relic abundances of all $\chi$ candidates from tables 1 and 2 in the mass range of $0.5 \mathrm{TeV}<m_{\chi}<5 \mathrm{TeV}$ with the $Y_{p}$ bound estimated in this way is shown in figure 11 (left hand side). Notice the almost discontinuous drop of the abundance for some representations. This is due to the fact that for colored multiplets featuring a neutral component there is a qualitative change of behaviour when the lifetime of the particle becames smaller than the annihilation lifetime of the associated hadronic bound state (cf. figure 10). We observe that all the $\chi$ are consistent with the $Y_{p}$ constraint. Also, most of the candidates have lifetimes shorter than $\sim 10 \mathrm{~s}$ (for $\Lambda_{\text {eff }} \sim 10^{15} \mathrm{GeV}$ ), so that no further bounds from BBN processes at later times can be derived. The only exceptions are the cases in table 6 where the lightest $\chi$ component can only decay through long cascades involving off-shell heavier components and $W$ bosons as well as $(1,7,0)_{S}$ decaying exclusively through loop-induced processes. For these cases the $\mathrm{D} / \mathrm{H}$ bound applies as shown in figure 11 (right hand side). In particular, while all the colored multiplets (including those decaying with cascades) are consistent with this constraint due to their low relic abundance after the second stage of strong annihilations, all the long-lived uncolored cases are in general constrained. In the relevant region of relic abundances and lifetimes, the bound turns out to be insensitive to the exact $\chi$ relic abundance or decay mode and so even our crude estimates suffice to extract fairly robust lower bounds on $\chi$ masses. They are shown in table 7 for a fixed value of $\Lambda_{\text {eff }}=10^{15} \mathrm{GeV}$, while the $\Lambda_{\text {eff }}$ dependence is shown explicitly in figure 12 . 


\begin{tabular}{|c|c|c|c|}
\hline Spin & $\chi$ & $Q_{\mathrm{LP}}$ & Mass bound $[\mathrm{GeV}]$ \\
\hline 0 & $(1,2,5 / 2)$ & 3 & 790 \\
0 & $(1,5,1)$ & 3 & 920 \\
0 & $(1,5,2)$ & 3,4 & 530,1900 \\
0 & $(1,7,0)$ & $0,1,2,3$ & $\gg 5000$ \\
\hline
\end{tabular}

Table 7. BBN bounds on the masses of long lived $\chi$ multiplet components assuming fixed $\Lambda_{\text {eff }}=$ $10^{15} \mathrm{GeV}$.

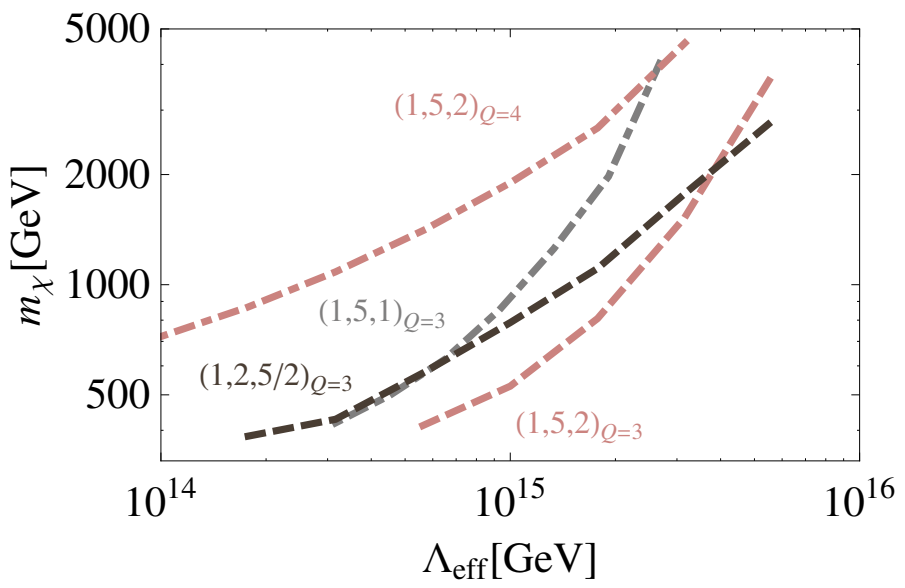

Figure 12. Dependence of the D/H bound on the $\chi$ mass as a function of the EFT cut-off scale $\Lambda_{\text {eff }}$ suppressing the relevant $d=5$ decay mediating operators. See text for details.

We finally note that it is close to these $\mathrm{D} / \mathrm{H}$ exclusion bounds where the primordial Lithium problem might be addressed by the presence of $\chi$ listed in the first three rows of table 7. A detailed exploration of this possibility goes however well beyond the scope of the present analysis and we leave it for future study.

\section{Collider phenomenology}

In this section, we discuss the collider phenomenology of the new states and give bounds on the masses of the new particles. The states of table 1 can be ordered into two classes: the ones which can decay by renormalizable interactions and the ones which decay via effective operators. In case the states decay via renormalizable interactions, they can be detected via their decay products. We will shortly comment on the renormalizable cases in section 5.1, mostly referring to the existing literature. If they only decay via effective operators they are rather long-lived and can eventually leave the detector before decaying. If the new particles are uncolored, the signature depends on whether the particle is charged or not. A summary of the different mass bounds for the uncolored cases can be found in table 8 .

All the extra colored states, given in table 2 , can only decay via $d=5$ operators and are hence long-lived. They hadronize and build exotic new mesons or baryons. We will discuss them in section 5.4. 


\begin{tabular}{|c|c|c|c|}
\hline Spin & $\chi$ & $Q_{\mathrm{LP}}$ & Mass bound $[\mathrm{GeV}]$ \\
\hline 0 & $(1,2,3 / 2)$ & 1,2 & 430,420 \\
0 & $(1,2,5 / 2)$ & 2,3 & 460,460 \\
0 & $(1,5,0)$ & $0,1,2$ & $75,500,600$ \\
0 & $(1,5,1)$ & $-1,0,1,2,3$ & $640,50^{\star}(85), 320,490,600$ \\
0 & $(1,5,2)$ & $0,1,2,3,4$ & $85,530,410,500,570$ \\
0 & $(1,7,0)$ & $0,1,2,3$ & $75,500,600,670$ \\
$1 / 2$ & $(1,4,1 / 2)$ & -1 & 860 \\
$1 / 2$ & $(1,4,3 / 2)$ & 0 & 90 \\
$1 / 2$ & $(1,5,0)$ & 0 & 95 \\
\hline
\end{tabular}

Table 8. LHC-I/LEP summary bounds for uncolored accidental matter multiplets decaying via $d \geq 5$ operators. Bounds on the neutral particles are given under the assumption of very small mass splitting to the $|Q|=1$ component. The exclusion bound in braces corresponds to the case where the next-to-LP has $Q=-1$ instead of $Q=1$. ${ }^{\star}$ A stronger exclusion bound, depending on the size of the portal coupling $\alpha$ (see eq. (2.5)), can be obtained from the Higgs data.

The production of the new exotic fermions and scalars proceeds via Drell-Yan processes. Throughout this section, we use the LO Drell-Yan production cross sections. Formulae are given e.g. in ref. [37]. The cross section for scalars is in general more than one order of magnitude smaller than for fermions, which explains the lower exclusion bounds on the scalars.

\subsection{Renormalizable cases}

Among the extra multiplets which preserve the flavor group of the SM, compatibly with cosmology and a cut-off scale of $\Lambda_{\text {eff }} \approx 10^{15} \mathrm{GeV}$, we identified four states which decay via renormalizable interactions, namely $(1,1,0)_{S},(1,3,0)_{S},(1,4,1 / 2)_{S}$ and $(1,4,3 / 2)_{S}$. In all of these cases, the new state acquires a VEV. With the only exception of the SM singlet, these VEVs must be small in order to comply with EW precision measurements. For $\mathcal{O}(1)$ couplings in the scalar potential and barring fine-tunings this implies $m_{\chi} \gtrsim 2-20 \mathrm{TeV}$, cf. section 2.2.1.

The gauge singlet can sizeably mix with the Higgs boson. Such mixing is constrained by the current Higgs data, see e.g. ref. [86]. The triplet and quadruplet scalar multiplets can only have a very small mixing with the Higgs boson due to its effects on EW precision observables. Nevertheless, their charged components can modify the Higgs to $\gamma \gamma$ and to $Z \gamma$ rates by their loop contributions. Whether these loop contributions suppress or enhance the diphoton rates depends on the sign of the couplings in the scalar potential [87, 88]. Finally, masses of the new neutral scalars below $62.5 \mathrm{GeV}$ can be probed by the invisible Higgs boson width. For more details see section 2.2.2.

Low masses of the triplets and quadruplets can be constrained by the $Z$ width (see section 5.3 for more details). Their charged components can also be directly detected. They 
decay into vector bosons or via cascades into vector bosons and the (off-shell) neutral components of the multiplet. The coupling to two vector bosons is proportional to the VEV of the multiplet. Apart from searches for singly charged Higgs bosons, searches for multiple charged Higgs bosons can provide a distinctive probe for large scalar multiplets. By now, searches for doubly charged Higgs bosons [89, 90] have only been performed for decays of the charged scalars into fermions, as in e.g. the case for models with $Y=1$ triplets [91] and quadruplets with additional vector-like matter for seesaw mass generation of neutrinos $[92,93]$. Bounds on doubly charged Higgs bosons decaying to $W^{ \pm} W^{ \pm}$can be obtained by reinterpreting SUSY searches for dileptons, missing energy and jets, and can exclude masses of the doubly charged scalars up to roughly $200 \mathrm{GeV}$ at $\sqrt{s}=7 \mathrm{TeV}$ for $\mathrm{SU}(2)_{L}$ triplets [94].

\subsection{Colorless and charged LP}

Charged stable particles will undergo charge exchange with the detector material. For masses larger than $100 \mathrm{GeV}$ the time of flight till to the outer detector is significantly larger than for lighter objects such as muons. They can hence be distinguished by their longer time of flight and by their anomalous energy loss in the detector. The energy loss in the detector is described by the Bethe-Bloch formula and depends on the speed and the charge of the particle. Searches for such ionizing tracks have been performed in refs. [95108]. The strongest bounds come from the CMS search of ref. [108], where the exclusion limits on the production cross sections of fractionally, singly and multiply charged particles are presented assuming vanishing quantum numbers under $\mathrm{SU}(2)_{L}$. Hence, in order to use the results of ref. [108] they need to be recast. Reference [109] gives tabulated efficiency values in terms of the transverse momentum, the pseudo rapidity and the velocity $\beta$ of the heavy charged particle. These can be used to reinterpret the results of ref. [108] without running a full detector simulation.

In order to compute the efficiencies, the models were implemented into Madgraph 5 [41] with the help of FeynRules [42]. The cross sections, computed at LO at the scale $Q=\sqrt{\hat{s}}$ using the MSTW2008 [110] parton distribution functions, were than rescaled by a factor accounting for the change in the efficiencies with respect to the cases considered in ref. [108].

We find that the efficiencies for fermions with non-vanishing $\mathrm{SU}(2)_{L}$ quantum numbers barely change compared to the case with $T_{3}=0$. The exclusion limits from ref. [108] on the cross section can hence be applied naively. For scalars, the efficiencies change slightly compared to the fermions. For masses of $300 \mathrm{GeV}$ the efficiency is slightly smaller than the one for fermions, for $800 \mathrm{GeV}$ it is roughly $15 \%$ larger. For the cases we considered, the efficiency for scalars is always within $3 \%$ of the case with vanishing quantum $\mathrm{SU}(2)_{L}$ quantum numbers.

In order to derive exclusion limits for the scalars, we adopt the following procedure. We compute the cross section and derive an approximate bound using the 95\% C.L. upper limits given in ref. [108]. With this approximate bound at hand, we compute at the naive bound the efficiencies for the scalar and compare it to the efficiencies of a fermion with $T_{3}=0$. The results can then be recast by the appropriate factor. We note, however, that such refined bounds are in good agreement with the results obtained naively. 
Even though the efficiency values in ref. [109] are given for the singly charged analysis only, we apply the same procedure to the $|Q|>1$ case to check whether also here, the naive method gives sensible results, as the basic cuts in both analysis are the same. Indeed we find also here that within the precision of our results, the naive estimate is very good. The results can be found in table 8 . The limits for charged fermions are stronger than for scalars due to the larger production cross section. The weakest exclusion limits are obtained for $T_{3}=0$.

Before concluding this subsection let us mention the recently approved LHC experiment MoEDAL [111, 112], whose target is the study of new physics phenomena (e.g. magnetic monopoles) which manifest themselves through the presence of highly-ionizing particles. In particular, the nuclear track detectors of MoEDAL are sensitive to particles with $|Q| / \beta \gtrsim 5$, where $Q$ is the charge and $\beta$ is the velocity of the particle in units of the speed of light. For our framework, with $Q$ ranging from 1 to 4 (cf. table 8), the discovery potential of the MoEDAL experiment will be relevant at low values of the $\beta$ distribution.

\subsection{Colorless and neutral LP}

The search for stable (on detector scale) neutral and colorless particles is very challenging at the LHC. Limits can either be set directly on the mass of the neutral particle as e.g. by mono-x searches or from constraints on the invisible $Z$ width or, indirectly, by giving bounds on the mass of the second lightest particle of the multiplet, such as in disappearing track signatures. Let us discuss in turn all these possibilities:

- Mono-x searches: neutral stable particles are searched for at the LHC in mono-x searches, in which large missing energy is accompanied by a radiation of an additional high-energetic particle $\mathrm{x}$, where " $\mathrm{x}$ " can stand for a jet, a photon, a $W$ or $Z$ boson, a top quark or a Higgs bosons. Nevertheless, we find that the monojet searches of ref. [113], which potentially have the strongest reach [114], are not sensitive to our states yet. Similar results were found for instance in ref. [7], in the case of a fermionic $(1,3,0)$ multiplet. Monojet searches can, however, become sensitive at $14 \mathrm{TeV}$ [7].

- Invisible $Z$ width: at LEP, the $Z$ boson width was determined with high accuracy [115, 116]. This measurements set a tight bound on new physics contributions to the invisible $Z$ width at the level of $\Gamma_{\mathrm{inv}}^{\text {new }}<2 \mathrm{MeV}$. This hence excludes charged particles, or particles with non-trivial $\mathrm{SU}(2)_{L}$ quantum numbers, up to the kinematic bound for the $Z \rightarrow \chi \chi^{\dagger}$ decay, meaning that masses $m_{\chi} \lesssim 45 \mathrm{GeV}$ are excluded.

- Disappearing tracks: disappearing tracks can be observed at the LHC if a rather longlived charged particle decays within the sensitive volume into a neutral particle and a soft pion, which is not detected. The strongest limits on these searches [117], are sensitive to lifetimes of the charged particle between $0.1 \mathrm{~ns}$ and $10 \mathrm{~ns}$. We checked whether the typical lifetimes for our particles lie within this range. It turns out, however, that for all fermionic states of table 1 with a lightest neutral state, the mass splitting between the neutral component and the charged component is always so large, that the lifetime is smaller than $0.1 \mathrm{~ns}$ (cf. figure 5). This is due to the 
fact that the radiative mass splitting increases with the hypercharge and the $\mathrm{SU}(2)_{L}$ quantum number. For the scalar states featuring a lightest neutral component and which do not decay through renormalizable interactions, the same argument holds. In addition, the mass splitting does not need to be purely radiative but a larger mass splitting can also stem from the potential term.

- LEP bounds on charginos: the LEP experiments set bounds on charginos that are nearly mass degenerate with the lightest neutralino. These bounds can be reinterpreted for our purposes in order to derive limits on the mass of the lightest neutral particles, since we showed in section 2.3 that the next-to-LP has always charge $Q_{\mathrm{LP}} \pm 1$. References [118-121] cover a mass splitting $\Delta m$ between $200 \mathrm{MeV} \lesssim \Delta m \lesssim 5 \mathrm{GeV}$ and are based on soft events with an initial state radiated photon. In order to estimate the limits for the case where the LP of the multiplet is neutral, we took the OPAL results of ref. [121]. There, the results were given in terms of a 95\% C.L. upper limit on the cross section. We implemented the models into MadGraph 5 with the help of FeynRules [42], computed the cross section values for the $|Q|=1$ charged component of the multiplet, and compared them to the given limits in ref. [121]. In order to verify this procedure, we computed the efficiencies for example points on parton level, using MadAnalysis [122]. For the fermionic states we found that the efficiencies are basically unchanged compared to the chargino case. For the scalars they turned out to be a bit reduced, which is however not relevant given the precision to which we estimate the bounds. The hence obtained limits on the charged components are given in table 8. At the accuracy we are working this essentially corresponds to the bounds on the neutral components, which are obtained after subtracting the small mass splitting.

For the case of the scalar $(1,5,1)$ multiplet, either the +1 charged or the -1 charged component can be the next-to-LP. These two cases lead to different exclusion bounds. The exclusion bound from the $Q=1$ state being the second-lightest component is much smaller due to the smaller production cross section, as this state corresponds to $T^{3}=0$. In such a case a stronger bound can come from the Higgs invisible width.

What about mass splittings larger than $5 \mathrm{GeV}$ not covered by the chargino search of ref. [121]? If the neutral LP of a scalar multiplet is the component with the smallest/largest isospin, then the mass splitting between the $|Q|=1$ next-to-LP and the neutral LP can also be larger than $5 \mathrm{GeV}$. This is not true for a generic value of the isospin $-j<I<j$, as for the neutral state to be the lightest a cancellation between the tree-level and radiative mass splitting is required. In particular, the only case in which we have to consider a mass splitting larger than $5 \mathrm{GeV}$ is for $(1,5,2)_{S}$. Even in such a case, however, mass splittings larger than about $20 \mathrm{GeV}$ are excluded by EW precision observables (cf. section 2.2.1).

Searches for charginos decaying into neutralinos and $W$ bosons (with the $W$ s decaying hadronically, semileptonically or leptonically) and for $\Delta m>5 \mathrm{GeV}$ were performed 
also at LEP [123]. ${ }^{14}$ In the $(1,5,2)_{S}$ case, for mass splittings around $5 \mathrm{GeV}$ the estimated bounds on the $|Q|=1$ particle decaying to the neutral state turn out to be weaker (by roughly $10 \mathrm{GeV}$ ) than the ones given in table 8. For mass splittings larger than $15 \mathrm{GeV}$ the limits become tighter (by around $5 \mathrm{GeV}$ ). A more detailed analysis is however beyond the scope of this paper.

\subsection{Colored LP}

The description of long-lived colored particles is complicated by the effect of nonperturbative QCD interactions. In fact, in all the cases of table 2 the decay of the new states is induced by a $d=5$ operator and the lifetimes are long enough that heavy colored particles hadronize before decaying. The theoretical description of the hadron formation and of the nuclear interactions of such states with matter represents the main source of uncertainty. In this section, we will briefly describe the various steps for the path of our new states, from their production to their escape from the detector or, in case the initial velocity $\beta$ is small enough, to their eventual stopping and decay inside the detector. We finally conclude by looking at the recent LHC results that are tuned to the case of QCD bound states of SUSY particles with quarks and gluons. We refer to [124] for a review on various phenomenological aspects of stable massive particle at colliders.

Production. The production mechanism of the new particles in our framework is determined by the color quantum number and by the value of the mass. We are interested in the fundamental, the two indices symmetric and the adjoint representations of $\mathrm{SU}(3)_{c}$ and we denote such cases respectively as $C_{3}, C_{6}$ and $C_{8}$. At the renormalizable level the presence of a $\mathrm{U}(1)$ or a $Z_{2}$ accidental symmetry guarantees these states to be pair produced. The fate of the produced state crucially depends on the velocity at the production time. Relativistic particles will lose energy throughout the detector but eventually escape it, while slow particles will be stopped in the detector and decay at a later time. Typical velocity distributions are displayed in figure 13 . There is no significant difference between scalars and fermions. For higher center-of-mass energies and lower masses, $m_{\chi}$, higher velocities are more probable. Notice also that due to the normalization with respect to the total cross section there is no difference between the different color quantum numbers. Another issue that has to be considered for a complete description of the production mechanism is the Sommerfeld enhancement due to ladder exchange of gluons. This effect is relevant only for slowly produced states.

Hadronization. Once produced, a colored particle combines with quarks and gluons to form a colorless hadron state. For example, a color-triplet can form bound states such as $C_{3} \bar{q}$ or $C_{3} q_{1} q_{2}$, an octet state can form invariants such as $C_{8} \bar{q} q, C_{8} q_{1} q_{2} q_{2}$ or $C_{8} g$, while the sextuplet can hadronize in states of the form $C_{6} q g, C_{6} q \bar{q} q$ and $C_{6} \overline{q q}$. The probability for $C_{i}$ of hadronizing in a given bound state are rather uncertain,

\footnotetext{
${ }^{14}$ LHC searches for pair production of charginos, with the charginos decaying to $W$ s and neutralinos, are not yet sensitive to such low mass splittings.
} 

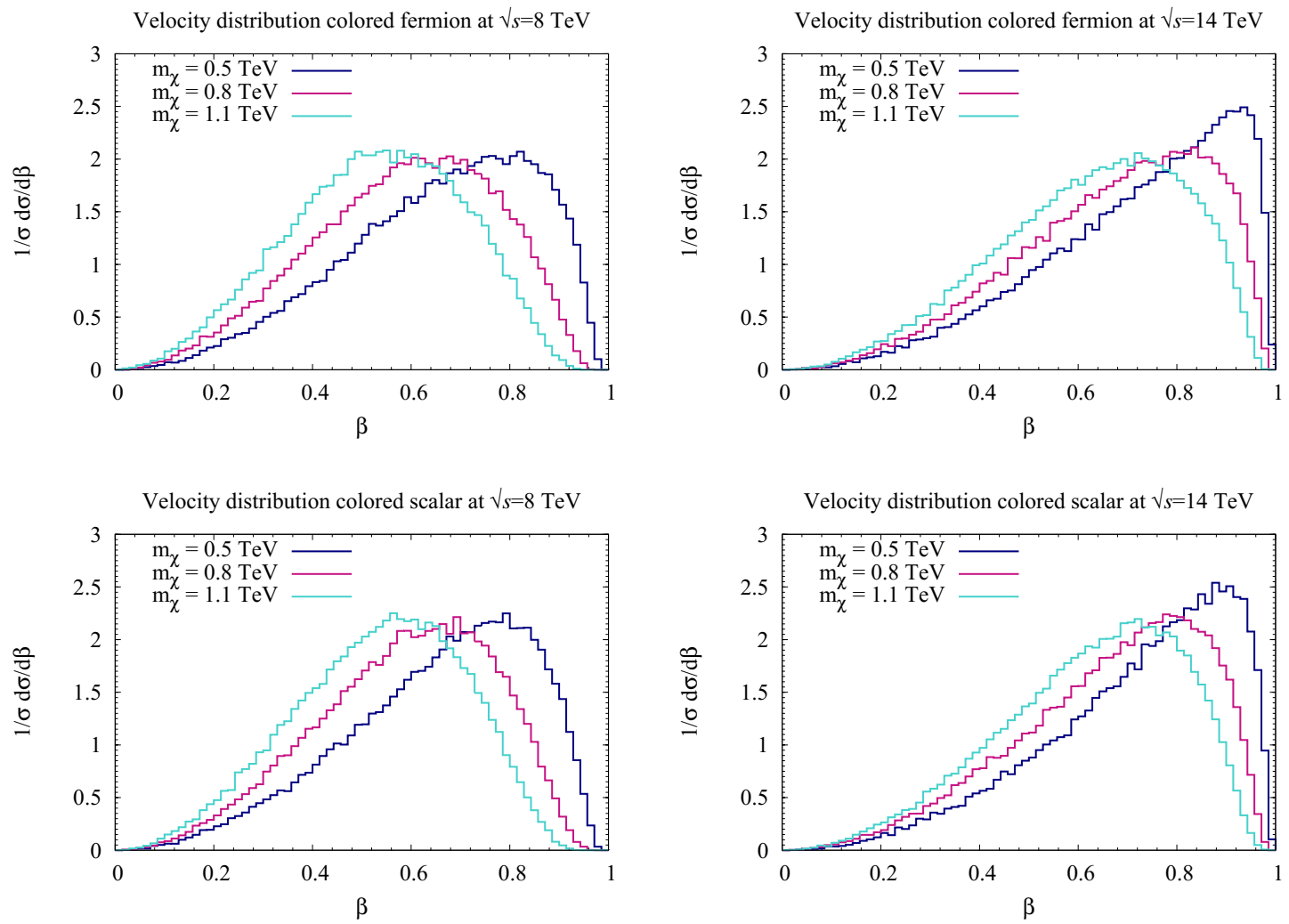

Figure 13. Velocity distribution normalized to the total cross section for colored fermonic states (top) and colored scalars (bottom). Left (right) panels correspond to $\sqrt{s}=8 \mathrm{TeV}(\sqrt{s}=14 \mathrm{TeV})$ for $m_{\chi}=500 \mathrm{GeV}$ (blue), $m_{\chi}=800 \mathrm{GeV}$ (pink) and $m_{\chi}=1.1 \mathrm{TeV}$ (light blue).

different models give quite different values. ${ }^{15}$ Bound states made of larger numbers of quarks and gluons are expected to be heavier [128] and, even if the hadronization in this channels could be non-negligible, the newly produced state could decay into a lighter one plus ordinary baryons and mesons, through QCD gauge interactions. Despite the fact that the hadronization processes are very uncertain, in some cases a detailed knowledge is not very important. As we are going to comment soon, nuclear conversions can wash out the information on the original hadron state at production.

Propagation through matter. As soon as produced and during their propagation, the long lived colored particles interact with the electrons, protons and neutrons present in the detector. The dominant interactions we consider here are the electromagnetic and the strong ones.

- Electromagnetic interactions: a particle with electric charge can interact with atomic electrons as well as with protons and neutrons in the nuclei. In the first case the net effect is the ionization of the atoms while interactions with atomic

\footnotetext{
${ }^{15}$ See for example the comparison between the string model adopted by Pythia [125] and the cluster model used in HERWIG [126, 127] in table 3 of [124].
} 
nuclei generate displacements of atoms from the lattice. In both cases the heavy long lived particle loses energy, however the energy loss $d E / d x$ from ionization is much larger than the one generated in the non-ionizing way.

The main role of the heavy elementary parton is to contribute to the electric charge of the hadron. Indeed the SM gauge quantum number and the requirement to obtain a color-singlet hadron state has an important influence on the total charge of the resulting bound state. We notice that considering all the cases in table 2, the resulting bound state has always integer charge. The most unfavourable situation, from the point of view of detection, happens when the resulting hadron is electromagnetically neutral.

- Strong interactions: before discussing the various kind of interactions between the heavy hadrons and the matter in the detector, let us clarify the role of the parton $C_{i}$ in the nuclear reactions. Due to their large mass, the wave-functions of the $C_{i}$ 's are expected to be highly localized as compared to those of the light constituents (quarks and gluons) that are spread in space as in ordinary QCD. From this observation we can draw the conclusions that the probability for the heavy parton $C_{i}$ to interact with matter is very low, while the typical cross section of the hadron with matter, being due to the effect of the light partons, is expected to be of the same order of those for pion scatterings.

Our heavy long-lived hadron can have elastic as well as inelastic reactions with nucleons. Elastic scatterings are not particularly relevant, indeed the energy loss is small because the long-lived hadron scatters on a much lighter target nucleus. Inelastic processes are instead those responsible for the slowing-down of the hadron. In an inelastic reaction it is also possible to exchange baryon and electric charge.

The importance of baryon exchange has been emphasized in [129], in these reactions a heavy hadron is transformed into another one with different baryon number. For the case of the gluino it has been argued that, bound states with null baryon charge ( $R$-mesons) are very efficiently converted into baryonic states in reactions like $\left(C_{8} d \bar{d}\right)+p \rightarrow \pi+\left(C_{8} u d d\right)$. The reverse reaction is suppressed mainly because of the mass split ordering of the various hadrons and by the low presence of pions as targetd in the detector material. As a consequence, early in the detector, mesons are converted into baryons.

Processes with charge exchange are particularly relevant for detecting the presence of the heavy parton. Indeed, tracks generated by the passage of electric particles can be easily detected, so it is important to understand the value of the electric charge of the hadron through all its travel in the calorimeter material. Even in the most pessimistic case of a hadron generated as a neutral bound state, reactions with charge exchange can covert it in a charged state that could be detected.

We finally mention that there are a series of phenomenological approaches to describe the strong interactions between heavy hadrons and matter [130-132]. 
Despite the fact they agree on several general qualitative aspects, they give rise to rather different quantitative results.

Stopping and decay. Depending on the $C_{i}$ mass, a non-negligible number of particles could stop in the detector. In our framework most of the states are supposed to decay within few seconds. This constitutes a really interesting possibility to understand the structure of the $d=5$ effective operators responsible for the decay. However, the detection of these processes represents a severe experimental challenge.

Having described the most important aspects of the phenomenology of our long lived particles, we now move to comment about direct searches. At the LHC, searches for longlived colored particles are performed in the context of $R$-hadrons, which are bound states of gluinos/ squarks and quarks/gluons. The $R$-hadrons can be detected by the longer time-of-flight to the outer detectors and their anomalous energy loss. Complementary to the searches relying simply on the longer time-of-flight and the anomalous energy loss, are searches for stopped $R$-hadrons. They are particularly suited for velocities $\beta \ll 1$.

In the former case, the strongest limits come from the CMS search of ref. [108], which excludes gluino masses up to $1276 \mathrm{GeV}$, if the fraction of gluinos hadronizing into $\tilde{g}-g$ bound states is 0.5 . If the fraction is equal to one, gluino masses are excluded up to $1250 \mathrm{GeV}$. In such a case the $R$-hadron is neutral in the inner tracker which leads to a smaller energy loss and hence a lower exclusion bound. In ref. [108] stop masses were excluded up to $935 \mathrm{GeV}(818 \mathrm{GeV})$. The exact exclusion bound depends on the modeling of the interactions of the stop with the detector material. The exclusion bound is here given for the so-called cloud model of ref. [129, 133] (the charged-suppressed model of ref. [134]). Very similar exclusion bounds were obtained in a recent ATLAS study [135] both for long-lived stops and gluinos.

In the search for out-of-time decays of stopped gluinos or squarks of ref. [136], gluinos are excluded up to masses of $880 \mathrm{GeV}$, assuming $\operatorname{BR}\left(\tilde{g} \rightarrow \tilde{\chi}_{1}^{0} g\right)=100 \%$ and a gluino lifetime between $1 \mu \mathrm{s}$ and $1000 \mathrm{~s}$. Stop masses are excluded up to $470 \mathrm{GeV}$ for $\operatorname{BR}\left(\tilde{t} \rightarrow \tilde{\chi}_{1}^{0} t\right)=100 \%$ and stop lifetimes between $1 \mu \mathrm{s}$ and $1000 \mathrm{~s}$. The exclusion bounds require that the neutralino mass is kinematically consistent with the used requirements on the energies of the gluon or respectively top decay products. The search furthermore assumes a cloud model for the $R$-hadron interactions. Reference [137] excludes sbottom masses up to $344 \mathrm{GeV}$ for $\operatorname{BR}\left(\tilde{b} \rightarrow \tilde{\chi}_{1}^{0} b\right)=100 \%$ for lifetimes between $1 \mu \mathrm{s}$ and $1000 \mathrm{~s}$.

The results on these searches of $R$-hadrons cannot straightforwardly be applied for our cases. In order to give exclusion bounds on our states a detailed study of the hadronization of the different states and a full detector simulation would be necessary. We expect that the exclusion limits not only depend strongly on the $\mathrm{SU}(3)_{c}$ quantum number, but they depend also on the charge and $\mathrm{SU}(2)_{L}$ quantum number. The charge influences the energy loss directly, whereas different $\mathrm{SU}(2)_{L}$ quantum numbers lead to different time intervals, in which the $R$-hadron propagates as a neutral particle in the detector due to pion exchange between the different members of an isomultiplet [138]. For stopped $R$-hadrons, in addition, the searches depend on the BRs. For our cases if the new exotic particle decays into a 
missing energy and jet signature, there are always several other operators allowing the particle to decay (cf. table 2), such that the corresponding BR likely deviates from 1.

A further discussion on the bounds on long-lived colored states is beyond the scope of this paper, as, even in the well-studied case of $R$-hadrons the exclusion bounds depend significantly on the modelling of the hadronization and the nuclear scattering model.

\section{Conclusions}

Low-energy tests of fundamental symmetries provide a powerful probe of new physics scales up to energies of about $10^{15} \mathrm{GeV}$. Given the accidental (B and L) and approximate (CP, flavor and custodial) symmetry structure of the SM, it is somewhat surprising that signals of physics beyond the SM (if it exists) have not been observed so far.

This last statement hinges on the theoretical prejudice that new physics effects at low energies can be described by a generic EFT, where the Wilson coefficients of the effective operators are $\mathcal{O}(1)$. There are, of course, exceptions to this point of view. The simplest one is maybe to allow for ultraweak couplings in the theory, so that the generic EFT power counting fails - an extreme example being the dissolution of the Weinberg operator when $\mathrm{RH}$ neutrinos are below the EW scale. On the other hand, the effective operators might not be there due to an exact symmetry of the Lagrangian in the full theory (as e.g. B - L in left-right symmetric models [139]) or they might be suppressed due to an approximate symmetry (as e.g. in minimal flavor violation [140]). In this paper, we explored yet another possibility: the quantum numbers of the new physics states below the EFT cut-off are such that by only requiring Lorentz and SM gauge invariance, the accidental and approximate symmetries of the SM are automatically preserved at the renormalizable level. The resulting new physics dynamics is practically invisible to low-energy indirect searches, and the only way of experimentally probing these scenarios is by direct production and detection of new particles at colliders. We hence focused on the phenomenological possibility that the new states lie within the kinematical reach of the LHC.

Barring few exceptions, the new matter multiplets are subject to extra accidental $Z_{2}$ or U(1) symmetries which forbid their decays at the renormalizable level. Whenever the LP in the multiplet is color- and charge-neutral, it forms a DM candidate [4-7]. Generally however, the extra multiplets will decay due to the presence of higher dimensional operators in the EFT. In the spirit of generic EFT we choose to work with a cut-off scale of $\Lambda_{\text {eff }} \approx$ $10^{15} \mathrm{GeV}$ which is large enough not to require any further protection mechanisms in the full theory and is moreover suggested by the observations of neutrino masses. The infinite set of possible states which satisfy the accidental symmetry conditions can then be reduced thanks to cosmological considerations. In particular, since the new states are long-lived, scenarios where the lightest component of the new multiplet is charged and/or colored are constrained by cosmological observations as well as by searches for exotic forms of matter on the Earth and in the Universe. The latter practically exclude all the cases where the charged and/or colored LP decays via $d>5$ operators.

Another handle in order to further reduce the list of possible states is the requirement that the theory remains perturbative up to the cut-off scale of the EFT. In particular, we 
required that no Landau poles are generated below $\Lambda_{\text {eff }} \approx 10^{15} \mathrm{GeV}$. As a byproduct of the perturbativity analysis we noticed that, due to accidental cancellations in the coefficients of the one-loop beta functions for the non-abelian gauge factors, two-loop corrections can become important and hence the one-loop determination of the Landau pole can be misleading. A typical example is given by the two minimal DM cases in eqs. (2.16)-(2.17). In this respect, we also pointed out the existence of a previously overlooked $d=5$ operator which is responsible for a fast decay of $(1,7,0)_{S}$, thus ruling out the scalar minimal DM candidate [44].

The final set of states which satisfy all the above constraints is collected in tables 1-2. For these, we studied current bounds on their masses coming from their potential effects on BBN as well as from their production and detection at colliders. In particular, we found that for most of the states decaying through $d=5$ operators and being thermally produced in the early Universe, their abundances are sufficiently diluted not to affect standard BBN. The notable exceptions are those uncolored cases, where the decay rates are either loop suppressed or proceed through long cascades leading to high-multiplicity final states (they are listed in table 7). At colliders, the lightest particle of the multiplets in tables 1-2 are, barring few exceptions, stable on the detector scale. For the color singlets we found that the current mass bounds are of few hundred $\mathrm{GeV}$ if the lightest particle of the multiplet is charged, whereas for neutral states the detection is more difficult and hence the bounds lie below $100 \mathrm{GeV}$ (cf. table 8). On the other hand, for colored multiplets the mass bounds strongly depend on the hadronization process and the nuclear interactions with the detector material.

A this point, a natural question to ask is the following: what is accidental matter good for? Who ordered that? Besides the case of minimal DM, we note that the scalar multiplets in tables 1-2 could easily improve the stability of the renormalizable Higgs potential. ${ }^{16}$ Close to the EFT cut-off, the potential can again be destabilized in cases where $d=5$ operators exist containing only scalars, namely $(1,5,0)_{S},(1,5,1)_{S},(1,5,2)_{S}$, $(1,7,0)_{S},(8,1,0)_{S},(8,1,1)_{S},(8,3,0)_{S}$ and $(8,3,1)_{S}$. However, at such large field values the whole tower of operators should be considered and stabilization is expected to be recovered via $d \geq 6$ operators. Finally, a charged thermal relic with $\tau_{\chi} \sim\left(10^{2}-10^{3}\right)$ s and abundance just below the $\mathrm{D} / \mathrm{H}$ bound (which can be achieved for some of the accidental matter states listed in table 7) may also help to resolve the standard BBN Lithium problems [74-79]. More generally, accidental matter should be seen as a purely phenomenological possibility. New physics might manifest itself in a way we were not expecting and thus the direct search strategies should cover diverse scenarios. In particular, the typical signature of accidental matter is the presence of charged/colored particles which are stable on the scale of particle detectors and which have no chances to be detected through indirect searches. Consequently, high-energy colliders will be the only means of probing such scenarios. New experiments in the near future (LHC-II, MoEDAL, etc) will have the capabilities to further explore their parameter space.

\footnotetext{
${ }^{16}$ On the other hand, we explicitly checked that none of the weak-scale accidental matter states improves on gauge coupling unification with respect to the SM.
} 
We end by noting that an improvement in $p$-decay bounds by an order of magnitude [141] or failure to observe neutrinoless double beta decay with inverse neutrino mass hierarchy in the next generation of experiments [142] would put some pressure on this setup. In particular, (i) if neutrino oscillation experiments were to confirm the inverse neutrino mass hierarchy, then our EFT setup predicts an observable neutrinoless double beta decay signal. Failure to observe one in the next generation of experiments would imply the presence of NP degrees of freedom below the EFT cut-off which couple to SM fermions. Finally, (ii) there is already a mild tension between the proton decay bounds and neutrino mass measurements, if one assumes a common EFT scale for both phenomena. This tension would be strengthened by future improvements in $p$-decay bounds or by a positive indication of a quasi-degenerate light neutrino spectrum, requiring a significant scale separation between the relevant $\mathrm{L}$ and $\mathrm{B}$ violating operators.

\section{Acknowledgments}

We thank Sergio Cecotti, Talal Ahmed Chowdhury, Ben Gripaios, Miha Nemevšek, Paolo Panci, Giovanni Marco Pruna and Christian Reuschle for useful discussions. This work was supported in part by the Slovenian Research Agency. The work of L.D.L. is supported by the Marie Curie CIG program, project number PCIG13-GA-2013-618439. L.D.L., R.G. and M.N. are grateful to the theoretical physics group of the Jožef Stefan Institute for hospitality and support during the development of this project. L.D.L. would like to thank the highenergy physics group of the University of Roma Tre for hospitality during the completion of this work. R.G. would like to thank the theoretical physics group of the University of Genova for hospitality during the completion of this work.

\section{A Two-loop Landau poles}

In this appendix we provide the RG evolution of the gauge couplings and study the emergence of the associated Landau poles. In this way one can set an upper bound on the dimensionality of the extra representation, by requiring that no Landau poles are generated below $\Lambda_{\mathrm{eff}} \approx 10^{15} \mathrm{GeV}$ (cf. the discussion in section 2.4).

The two-loop RG equation for the three gauge couplings $g_{i}(i=1,2,3)$, read

$$
\frac{\mathrm{d}}{\mathrm{d} t} \alpha_{i}^{-1}=-a_{i}-\frac{b_{i j}}{4 \pi} \alpha_{j},
$$

where $\alpha_{i}=\frac{g_{i}^{2}}{4 \pi}$ and $t=\frac{1}{2 \pi} \log \frac{\mu}{M_{Z}}$. The one- and two-loop beta function are [143] (no summation over $i$ )

$$
a_{i}=-\frac{11}{3} C_{2}\left(G_{i}\right)+\frac{4}{3} \sum_{F} \kappa S_{2}\left(F_{i}\right)+\frac{1}{3} \sum_{S} \eta S_{2}\left(S_{i}\right)
$$




$$
\begin{aligned}
b_{i j}=\left[-\frac{34}{3}\left(C_{2}\left(G_{i}\right)\right)^{2}+\sum_{F}\left(4 C_{2}\left(F_{i}\right)+\frac{20}{3} C_{2}\left(G_{i}\right)\right) \kappa S_{2}\left(F_{i}\right)\right. \\
\left.+\sum_{S}\left(4 C_{2}\left(S_{i}\right)+\frac{2}{3} C_{2}\left(G_{i}\right)\right) \eta S_{2}\left(S_{i}\right)\right] \delta_{i j} \\
+4\left[\sum_{F} \kappa C_{2}\left(F_{j}\right) S_{2}\left(F_{i}\right)+\sum_{S} \eta C_{2}\left(S_{j}\right) S_{2}\left(S_{i}\right)\right]
\end{aligned}
$$

where $G_{i}$ denotes the $i$-th gauge factor, $S_{2}$ and $C_{2}$ are the index (including multiplicity factors) and the quadratic Casimir of a given (fermionic $(F)$ or scalar $(S)$ ) irreducible representation; $\kappa=1, \frac{1}{2}$ for Dirac and Weyl fermions and $\eta=1, \frac{1}{2}$ for complex and real scalar fields, respectively. The Yukawa contribution in the two-loop beta function is neglected. In fact, the extra states we want to introduce do not couple with SM fermions, so that the Yukawa contribution does not grow with the dimensionality of the extra representation. Employing the GUT normalization for the abelian factor, we use the values $\alpha_{1}\left(m_{Z}\right)=0.016923, \alpha_{2}\left(m_{Z}\right)=0.03374$, and $\alpha_{3}\left(m_{Z}\right)=0.1173$ for the onset of the RG running $[17,144]$. For simplicity, the extra state $\chi$ is integrated in at $m_{Z}=91.188 \mathrm{GeV}$ [17]. The scaling of the Landau pole with $m_{\chi}$ is approximately linear.

For the cases where the $\mathrm{SM}$ is extended with a representation charged under $\mathrm{SU}(3)_{c}$ and/or $\mathrm{SU}(2)_{L}$, the results are summarized in table 9 , which provide a useful reference for estimating the bound on the dimensionality of the extra representations, by requiring that no Landau poles are generated below a given scale.

The analysis has been repeated for all the states considered in this work, which can simultaneously transform under $\mathrm{SU}(3)_{c}$ and $\mathrm{SU}(2)_{L}$, and have a non-zero hypercharge as well. These include extra representations interacting with SM fields via $d=5$ operators (but which cannot decay into SM states via renormalizable interactions), for which the results are reported in table 10. Moreover, we investigated the Landau pole constraints for those extra scalar representations that can couple to the Higgs boson at the renormalizable level (cf. table 5). Among them the only ones that do not couple to SM fermions at the renormalizable level and that survive the perturbativity criteria are the renormalizable cases of table 1 . In this respect, we mention a marginal case: $(1,6,1 / 2)_{S}$ for which $\Lambda_{\text {Landau }}^{2-\text { loop }}=6.6 \times 10^{13} \mathrm{GeV}$. Finally, we also checked the possibility of having multiplets decaying via $d>5$ operators and whose neutral LP might be compatible with cosmological constraints. No cases beyond those of minimal DM (cf. table 1) and with a Landau pole above $10^{15} \mathrm{GeV}$ are found.

\section{B $\quad \mathrm{SU}(2)_{L}$ decompositions}

By denoting the generators in the fundamental representation of $\mathrm{SU}(2)_{L}$ as $T^{a}=\sigma^{a} / 2$ (with $\sigma^{a}$ being the Pauli matrices and $\left.a=1,2,3\right)$, we define their action on the $(2 j+1)$ dimensional completely symmetric tensor $\chi_{i_{1} i_{2} \ldots i_{2 j}}\left(i_{1}, i_{2}, \ldots, i_{2 j}=1,2\right)$ as

$$
\delta^{a}\left(\chi_{i_{1} i_{2} \ldots i_{2 j}}\right)=T_{i_{1} k}^{a} \chi_{k i_{2} \ldots i_{2 j}}+T_{i_{2} k}^{a} \chi_{i_{1} k \ldots i_{2 j}}+\ldots+T_{i_{2 j} k}^{a} \chi_{i_{1} i_{1} \ldots k}
$$




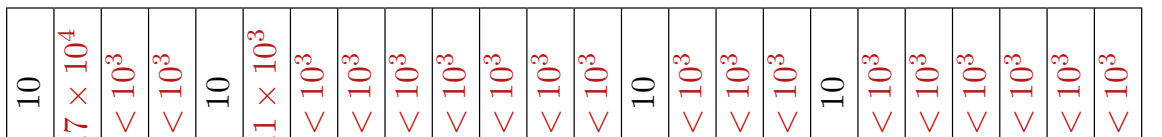

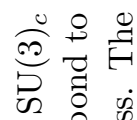
की 要 $\underbrace{\infty}_{0} \frac{0}{0} \frac{\pi}{0}$ 晃

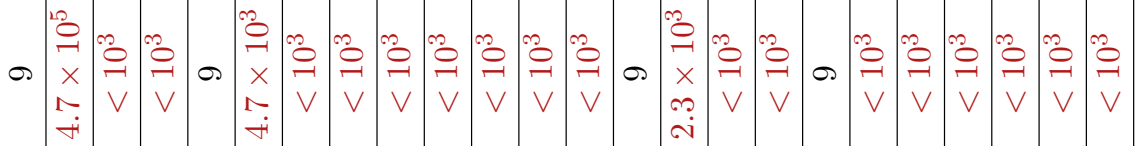

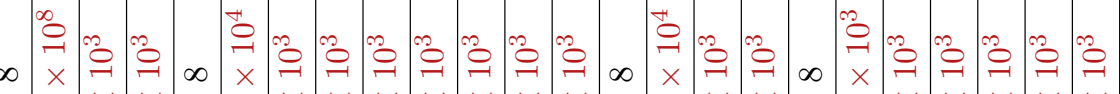

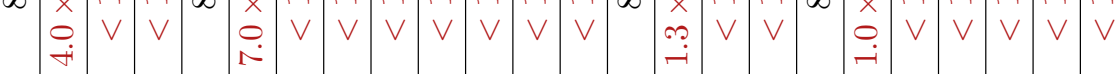

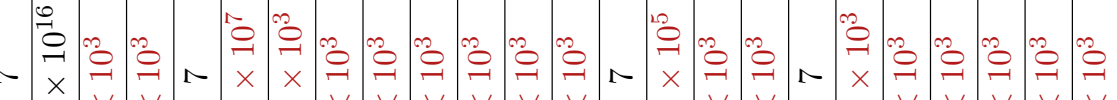

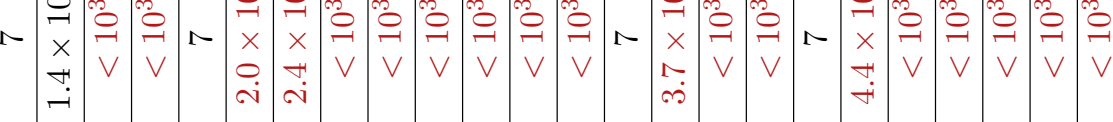

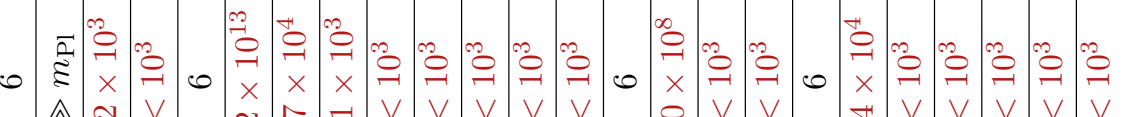

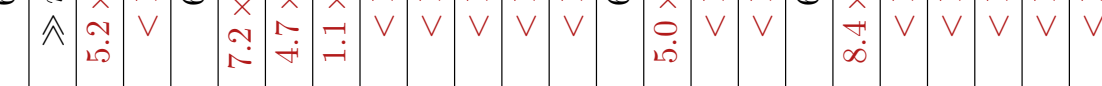

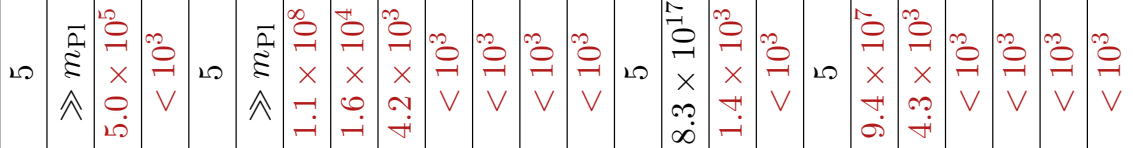
룽 $\frac{7}{1} \stackrel{2}{\circ}$ (N) है $\frac{7}{3}$ र $+\frac{\vec{B}}{\omega}$ $\exists$ : 要

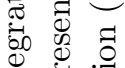
苛密:

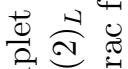

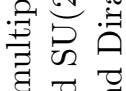
च $\bar{G}$

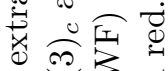
娄这。

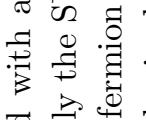

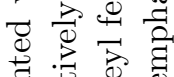

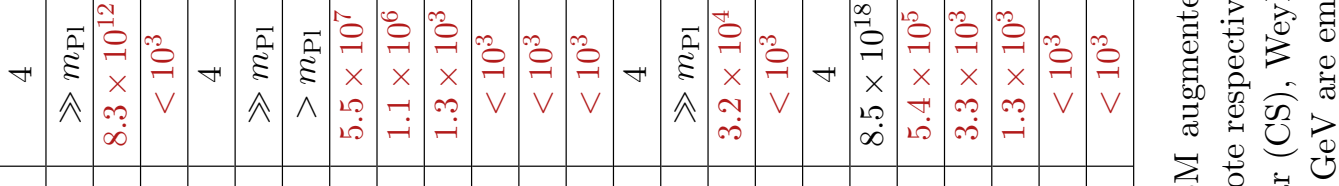

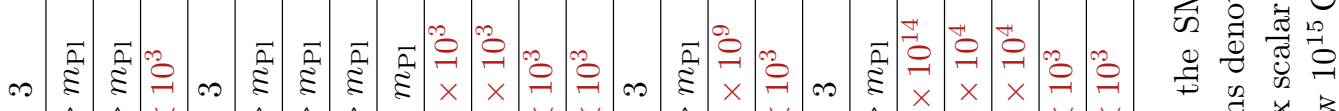

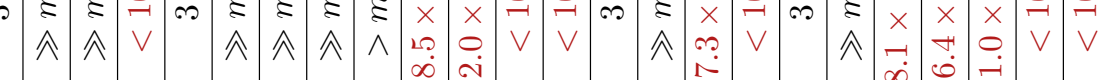

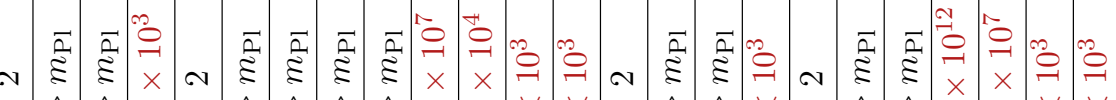

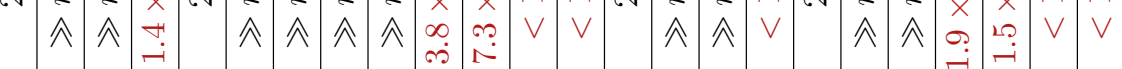

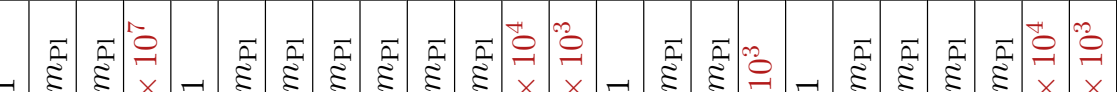

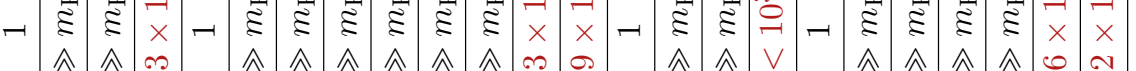

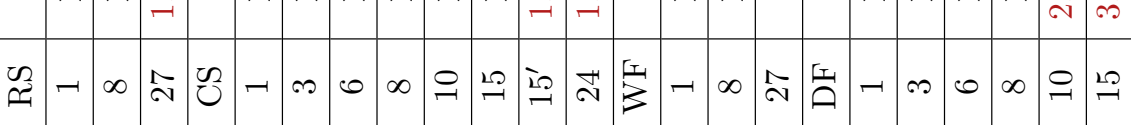

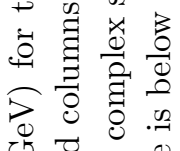
एँ कृ की है 웅 ש

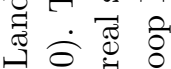
०ी 11 क

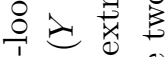
슨

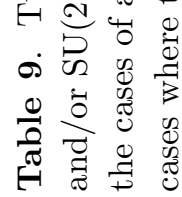




\begin{tabular}{|c|c|c|}
\hline Spin & $\chi$ & $\Lambda_{\text {Landaul }}^{2-\text { loov }}[$ \\
\hline 0 & $(1,2,3 / 2)$ & $\gg m_{\mathrm{Pl}}\left(g_{1}\right)$ \\
0 & $(1,2,5 / 2)$ & $\gg m_{\mathrm{Pl}}\left(g_{1}\right)$ \\
0 & $(1,5,0)$ & $\gg m_{\mathrm{Pl}}\left(g_{1}\right)$ \\
0 & $(1,5,1)$ & $\gg m_{\mathrm{Pl}}\left(g_{1}\right)$ \\
0 & $(1,5,2)$ & $3.5 \times 10^{18}\left(g_{1}\right)$ \\
0 & $(1,7,0)$ & $1.4 \times 10^{16}\left(g_{2}\right)$ \\
0 & $(3,1,5 / 3)$ & $\gg m_{\mathrm{Pl}}\left(g_{1}\right)$ \\
0 & $(\overline{3}, 2,5 / 6)$ & $\gg m_{\mathrm{Pl}}\left(g_{1}\right)$ \\
0 & $(\overline{3}, 2,11 / 6)$ & $5.5 \times 10^{19}\left(g_{1}\right)$ \\
0 & $(3,3,2 / 3)$ & $\gg m_{\mathrm{Pl}}\left(g_{1}\right)$ \\
0 & $(3,3,5 / 3)$ & $3.2 \times 10^{17}\left(g_{1}\right)$ \\
0 & $(3,4,1 / 6)$ & $\gg m_{\mathrm{Pl}}\left(g_{2}\right)$ \\
0 & $(\overline{3}, 4,5 / 6)$ & $\gg m_{\mathrm{Pl}}\left(g_{2}\right)$ \\
0 & $(\overline{6}, 2,1 / 6)$ & $\gg m_{\mathrm{Pl}}\left(g_{1}\right)$ \\
0 & $(6,2,5 / 6)$ & $\gg m_{\mathrm{Pl}}\left(g_{1}\right)$ \\
0 & $(\overline{6}, 2,7 / 6)$ & $\gg m_{\mathrm{Pl}}\left(g_{1}\right)$ \\
0 & $(6,2,11 / 6)$ & $4.0 \times 10^{12}\left(g_{1}\right)$ \\
0 & $(\overline{6}, 4,1 / 6)$ & $5.5 \times 10^{7}\left(g_{2}\right)$ \\
0 & $(6,4,5 / 6)$ & $5.0 \times 10^{7}\left(g_{2}\right)$ \\
0 & $(8,1,0)$ & $\gg m_{\mathrm{Pl}}\left(g_{1}\right)$ \\
0 & $(8,1,1)$ & $\gg m_{\mathrm{Pl}}\left(g_{1}\right)$ \\
0 & $(8,3,0)$ & $\gg m_{\mathrm{Pl}}\left(g_{1}\right)$ \\
0 & $(8,3,1)$ & $1.0 \times 10^{17}\left(g_{1}\right)$ \\
0 & $(27,1,0)$ & $1.3 \times 10^{7}\left(g_{3}\right)$ \\
$1 / 2$ & $(1,3,2)$ & $1.4 \times 10^{13}\left(g_{1}\right)$ \\
$1 / 2$ & $(1,4,1 / 2)$ & $8.1 \times 10^{18}\left(g_{2}\right)$ \\
$1 / 2$ & $(1,4,3 / 2)$ & $2.7 \times 10^{15}\left(g_{1}\right)$ \\
$1 / 2$ & $(\overline{3}, 3,4 / 3)$ & $9.3 \times 10^{10}\left(g_{1}\right)$ \\
$1 / 2$ & $(\overline{3}, 3,5 / 3)$ & $1.6 \times 10^{8}\left(g_{1}\right)$ \\
$1 / 2$ & $(3,4,1 / 6)$ & $5.4 \times 10^{5}\left(g_{2}\right)$ \\
$1 / 2$ & $(\overline{3}, 4,5 / 6)$ & $5.3 \times 10^{5}\left(g_{2}\right)$ \\
$1 / 2$ & $(3,4,7 / 6)$ & $5.2 \times 10^{5}\left(g_{2}\right)$ \\
$1 / 2$ & $(6,1,1 / 3)$ & $\gg m_{\mathrm{Pl}}\left(g_{1}\right)$ \\
$1 / 2$ & $(\overline{6}, 1,2 / 3)$ & $\gg m_{\mathrm{Pl}}\left(g_{1}\right)$ \\
$1 / 2$ & $(\overline{6}, 2,1 / 6)$ & $1.9 \times 10^{12}\left(g_{3}\right)$ \\
$1 / 2$ & $(8,1,1)$ & $4.0 \times 10^{16}\left(g_{1}\right)$ \\
$1 / 2$ & $(8,2,1 / 2)$ & $1.5 \times 10^{7}\left(g_{3}\right)$ \\
$1 / 2$ & $(\overline{15}, 1,1 / 3)$ & $3.2 \times 10^{3}\left(g_{3}\right)$ \\
$1 / 2$ & $(15,1,2 / 3)$ & $3.2 \times 10^{3}\left(g_{3}\right)$ \\
$1 / 2$ & $(15,2,1 / 6)$ & $3.6 \times 10^{2}\left(g_{3}\right)$ \\
\hline & & \\
\end{tabular}

Table 10. List of extra multiplets which can decay into SM particles via $d=5$ operators (states decaying via $d=4$ operators have been already subtracted) and corresponding two-loop Landau poles evaluated by integrating in the new states at $m_{Z}$ (fields with zero hypercharge are understood to be real). In the third column, the symbol in the bracket stands for the gauge coupling responsible for the emergence of the Landau pole. 
In general, we arrive at the following embedding of the properly normalized $T^{3}$ eigenstates:

$$
\begin{aligned}
\chi_{11 \ldots 1} & =\frac{1}{\sqrt{B_{2 j, 0}}} \chi^{j} \\
\chi_{11 \ldots 2} & =\frac{1}{\sqrt{B_{2 j, 1}}} \chi^{j-1} \\
\vdots & \\
\chi_{12 \ldots 2} & =\frac{1}{\sqrt{B_{2 j, 2 j-1}}} \chi^{-j+1} \\
\chi_{22 \ldots 2} & =\frac{1}{\sqrt{B_{2 j, 2 j}}} \chi^{-j},
\end{aligned}
$$

where the superscripts denote the $T^{3}$ eigenvalue, $B_{n, k}$ is the binomial factor $B_{n, k}=\frac{n !}{k !(n-k) !}$ and the normalization of the states is such that

$$
\chi^{* i_{1} i_{2} \ldots i_{2 j}} \chi_{i_{1} i_{2} \ldots i_{2 j}}=\left|\chi^{j}\right|^{2}+\left|\chi^{j-1}\right|^{2}+\ldots+\left|\chi^{-j+1}\right|^{2}+\left|\chi^{-j}\right|^{2} .
$$

Let us consider, for instance, the case of the $\mathrm{SU}(2)_{L}$ Higgs doublet:

$$
\begin{aligned}
& H_{1}=H_{+} \\
& H_{2}=H_{0},
\end{aligned}
$$

where the electric charge eigenstates are obtained through the formula $Q=T^{3}+Y$. In particular, since in the unitary gauge: $H_{+}=0, \operatorname{Im} H_{0}=0$ and $\operatorname{Re} H_{0}=\frac{1}{\sqrt{2}}(v+h)$, whenever the Higgs doublet is contained in the effective operator responsible for the decay of $\chi$, it might happen that not all of the components of $\chi$ can directly decay through the effective operator. In the following, we provide the $\mathrm{SU}(2)_{L}$ decomposition for the three uncoloured multiplets whose decay, depending on the mass spectrum, might proceed via off-shell cascades (cf. table 6):

$\chi=(1,2,5 / 2)_{S}:$

- $\mathrm{SU}(2)_{L}$ embedding:

$$
\begin{aligned}
& \chi_{1}=\chi_{+3} \\
& \chi_{2}=\chi_{+2} .
\end{aligned}
$$

- Operator:

$$
\mathcal{O}_{1}=\chi^{* i} e^{c} e^{c} H_{i}
$$

- Decomposition in the unitary gauge:

$$
O_{1}=\frac{1}{\sqrt{2}} \chi_{-2}^{*} e^{c} e^{c}(v+h) .
$$

Notice that $\chi_{+3}$ does not couple directly to SM particles. Hence, whenever it is the LP it will decay through an off-shell emission of $\chi_{+2}$ (cf. figure 6). 
$\chi=(1,5,1)_{S}:$

- $\mathrm{SU}(2)_{L}$ embedding:

$$
\begin{aligned}
& \chi_{1111}=\chi_{+3} \\
& \chi_{1112}=\frac{1}{\sqrt{4}} \chi_{+2} \\
& \chi_{1122}=\frac{1}{\sqrt{6}} \chi_{+1} \\
& \chi_{1222}=\frac{1}{\sqrt{4}} \chi_{0} \\
& \chi_{2222}=\chi_{-1} .
\end{aligned}
$$

- Operator:

$$
O_{1}=\chi^{* i j k l} H_{i} H_{j} H_{k} H^{* l^{\prime}} \epsilon_{l l^{\prime}} .
$$

- Decomposition in the unitary gauge:

$$
O_{1}=\frac{1}{8} \chi_{0}^{*}(v+h)^{4} .
$$

Notice that only $\chi_{0}$ can directly decay through $O_{1}$. If $\chi_{0}$ is not the LP in the multiplet, the charged LP will cascade decay through off-shell components which end up into $\chi_{0}$.

$\chi=(1,5,2)_{S}:$

- $\mathrm{SU}(2)_{L}$ embedding:

$$
\begin{aligned}
\chi_{1111} & =\chi_{+4} \\
\chi_{1112} & =\frac{1}{\sqrt{4}} \chi_{+3} \\
\chi_{1122} & =\frac{1}{\sqrt{6}} \chi_{+2} \\
\chi_{1222} & =\frac{1}{\sqrt{4}} \chi_{+1} \\
\chi_{2222} & =\chi_{0} .
\end{aligned}
$$

- Operator:

$$
O_{1}=\chi^{* i j k l} H_{i} H_{j} H_{k} H_{l} .
$$

- Decomposition in the unitary gauge:

$$
O_{1}=\frac{1}{4} \chi_{0}^{*}(v+h)^{4} .
$$

Similarly to the previous case, only $\chi_{0}$ can decay through $O_{1}$, while the charged components decay through off-shell cascades. 
Open Access. This article is distributed under the terms of the Creative Commons Attribution License (CC-BY 4.0), which permits any use, distribution and reproduction in any medium, provided the original author(s) and source are credited.

\section{References}

[1] G. Isidori, Y. Nir and G. Perez, Flavor physics constraints for physics beyond the standard model, Ann. Rev. Nucl. Part. Sci. 60 (2010) 355 [arXiv:1002.0900] [INSPIRE].

[2] V. Cirigliano and M.J. Ramsey-Musolf, Low energy probes of physics beyond the standard model, Prog. Part. Nucl. Phys. 71 (2013) 2 [arXiv:1304.0017] [INSPIRE].

[3] J.F. Kamenik, Flavor constraints on new physics, Mod. Phys. Lett. A 29 (2014) 1430021.

[4] M. Cirelli, N. Fornengo and A. Strumia, Minimal dark matter, Nucl. Phys. B 753 (2006) 178 [hep-ph/0512090] [INSPIRE].

[5] M. Cirelli, A. Strumia and M. Tamburini, Cosmology and astrophysics of minimal dark matter, Nucl. Phys. B 787 (2007) 152 [arXiv:0706.4071] [InSPIRE].

[6] M. Cirelli and A. Strumia, Minimal dark matter: model and results, New J. Phys. 11 (2009) 105005 [arXiv: 0903.3381] [INSPIRE].

[7] M. Cirelli, F. Sala and M. Taoso, Wino-like minimal dark matter and future colliders, JHEP 10 (2014) 033 [Erratum ibid. 1501 (2015) 041] [arXiv:1407.7058] [INSPIRE].

[8] J. Jaeckel, A force beyond the standard model - Status of the quest for hidden photons, Frascati Phys. Ser. 56 (2012) 172 [arXiv:1303.1821] [INSPIRE].

[9] P. Van Nieuwenhuizen, Supergravity, Phys. Rept. 68 (1981) 189 [INSPIRE].

[10] H.P. Nilles, Supersymmetry, supergravity and particle physics, Phys. Rept. 110 (1984) 1 [INSPIRE].

[11] S. Willenbrock, Symmetries of the standard model, hep-ph/0410370 [INSPIRE].

[12] S.S. AbdusSalam and T.A. Chowdhury, Scalar representations in the light of electroweak phase transition and cold dark matter phenomenology, JCAP 05 (2014) 026 [arXiv:1310.8152] [INSPIRE].

[13] M. Pospelov and A. Ritz, Electric dipole moments as probes of new physics, Annals Phys. 318 (2005) 119 [hep-ph/0504231] [INSPIRE].

[14] S. Inoue, M.J. Ramsey-Musolf and Y. Zhang, CP-violating phenomenology of flavor conserving two Higgs doublet models, Phys. Rev. D 89 (2014) 115023 [arXiv:1403.4257] [INSPIRE].

[15] T. Abe, J. Hisano, T. Kitahara and K. Tobioka, Gauge invariant Barr-Zee type contributions to fermionic EDMs in the two-Higgs doublet models, JHEP 01 (2014) 106 [arXiv:1311.4704] [INSPIRE].

[16] ACME collaboration, J. Baron et al., Order of magnitude smaller limit on the electric dipole moment of the electron, Science 343 (2014) 269 [arXiv:1310.7534] [INSPIRE].

[17] Particle Data Group collaboration, J. Beringer et al., Review of particle physics, Phys. Rev. D 86 (2012) 010001 [INSPIRE].

[18] J.F. Gunion, H.E. Haber, G.L. Kane and S. Dawson, The Higgs hunter's guide, Front. Phys. 80 (2000) 1. 
[19] J. Hisano and K. Tsumura, Higgs boson mixes with an $\mathrm{SU}(2)$ septet representation, Phys. Rev. D 87 (2013) 053004 [arXiv: 1301.6455] [InSPIRE].

[20] L. Lavoura and L.-F. Li, Making the small oblique parameters large, Phys. Rev. D 49 (1994) 1409 [hep-ph/9309262] [INSPIRE].

[21] A. Djouadi, The anatomy of electro-weak symmetry breaking. II. The Higgs bosons in the minimal supersymmetric model, Phys. Rept. 459 (2008) 1 [hep-ph/0503173] [INSPIRE].

[22] W.-F. Chang, J.N. Ng and J.M.S. Wu, Constraints on new scalars from the LHC $125 \mathrm{GeV}$ Higgs signal, Phys. Rev. D 86 (2012) 033003 [arXiv:1206.5047] [InSPIRE].

[23] I. Dorsner, S. Fajfer, A. Greljo and J.F. Kamenik, Higgs uncovering light scalar remnants of high scale matter unification, JHEP 11 (2012) 130 [arXiv:1208.1266] [INSPIRE].

[24] LHC Higgs cross section working group, https://twiki.cern.ch/twiki/bin/view/LHCPhysics/LHCHXSWG.

[25] CMS collaboration, Search for invisible decays of Higgs bosons in the vector boson fusion and associated ZH production modes, Eur. Phys. J. C 74 (2014) 2980 [arXiv:1404.1344] [INSPIRE].

[26] I. Doršner et al., New physics models facing lepton flavor violating Higgs decays at the percent level, JHEP 06 (2015) 108 [arXiv:1502.07784] [INSPIRE].

[27] T.A. collaboration, Search for the bb decay of the standard model Higgs boson in associated $W / Z H$ production with the ATLAS detector, arXiv:1207.0210 [INSPIRE].

[28] ATLAS collaboration, Measurements of Higgs boson production and couplings in diboson final states with the ATLAS detector at the LHC, Phys. Lett. B 726 (2013) 88 [Erratum ibid. B 734 (2014) 406] [arXiv: 1307.1427] [INSPIRE].

[29] T.A. collaboration, Updated coupling measurements of the Higgs boson with the ATLAS detector using up to $25 \mathrm{fb}^{-1}$ of proton-proton collision data, ATLAS-CONF-2013-012 (2013).

[30] ATLAS collaboration, Search for invisible decays of a Higgs boson produced in association with a Z boson in ATLAS, Phys. Rev. Lett. 112 (2014) 201802 [arXiv:1402.3244] [INSPIRE].

[31] CMS collaboration, Higgs to bb in the VBF channel, CMS-PAS-HIG-13-011 (2013).

[32] CMS collaboration, Search for Higgs boson production in association with a top-quark pair and decaying to bottom quarks or tau leptons, CMS-PAS-HIG-13-019 (2013).

[33] CMS collaboration, Measurement of Higgs boson production and properties in the $W W$ decay channel with leptonic final states, JHEP 01 (2014) 096 [arXiv:1312.1129] [INSPIRE].

[34] CMS collaboration, Measurement of the properties of a Higgs boson in the four-lepton final state, Phys. Rev. D 89 (2014) 092007 [arXiv: 1312.5353] [INSPIRE].

[35] CMS collaboration, Updated measurements of the Higgs boson at $125 \mathrm{GeV}$ in the two photon decay channel, CMS-PAS-HIG-13-001 (2013).

[36] CMS collaboration, Constraints on the Higgs boson width from off-shell production and decay to $Z Z \rightarrow$ llll and ll $\nu \nu$, CMS-PAS-HIG-14-002 (2014).

[37] E. Del Nobile, R. Franceschini, D. Pappadopulo and A. Strumia, Minimal matter at the Large Hadron Collider, Nucl. Phys. B 826 (2010) 217 [arXiv:0908.1567] [INSPIRE]. 
[38] G.F. Giudice, G. Isidori, A. Salvio and A. Strumia, Softened gravity and the extension of the standard model up to infinite energy, JHEP 02 (2015) 137 [arXiv:1412.2769] [INSPIRE].

[39] V. Barger, P. Langacker, M. McCaskey, M.J. Ramsey-Musolf and G. Shaughnessy, LHC phenomenology of an extended standard model with a real scalar singlet, Phys. Rev. D 77 (2008) 035005 [arXiv:0706.4311] [INSPIRE].

[40] R. Kleiss, W.J. Stirling and S.D. Ellis, A new Monte Carlo treatment of multiparticle phase space at high-energies, Comput. Phys. Commun. 40 (1986) 359 [INSPIRE].

[41] J. Alwall, M. Herquet, F. Maltoni, O. Mattelaer and T. Stelzer, MadGraph 5: going beyond, JHEP 06 (2011) 128 [arXiv:1106.0522] [INSPIRE].

[42] A. Alloul, N.D. Christensen, C. Degrande, C. Duhr and B. Fuks, FeynRules 2.0 - A complete toolbox for tree-level phenomenology, Comput. Phys. Commun. 185 (2014) 2250 [arXiv: 1310.1921] [INSPIRE].

[43] R. Grober, M. Muhlleitner, E. Popenda and A. Wlotzka, Light stop decays into Wb $\tilde{\chi}_{1}^{0}$ near the kinematic threshold, Phys. Lett. B 747 (2015) 144 [arXiv:1502.05935] [InSPIRE].

[44] E. Del Nobile, M. Nardecchia and P. Panci, On decaying minimal dark matter, work in progress.

[45] J. Kang, M.A. Luty and S. Nasri, The relic abundance of long-lived heavy colored particles, JHEP 09 (2008) 086 [hep-ph/0611322] [INSPIRE].

[46] B. Fields and S. Sarkar, Big-Bang nucleosynthesis (2006 Particle Data Group mini-review), astro-ph/0601514 [INSPIRE].

[47] W. Hu and J. Silk, Thermalization constraints and spectral distortions for massive unstable relic particles, Phys. Rev. Lett. 70 (1993) 2661 [INSPIRE].

[48] G.D. Kribs and I.Z. Rothstein, Bounds on longlived relics from diffuse gamma-ray observations, Phys. Rev. D 55 (1997) 4435 [Erratum ibid. D 56 (1997) 1822] [hep-ph/9610468] [INSPIRE].

[49] Fermi-LAT collaboration, M. Ackermann et al., Fermi LAT search for dark matter in gamma-ray lines and the inclusive photon spectrum, Phys. Rev. D 86 (2012) 022002 [arXiv: 1205.2739] [INSPIRE].

[50] S. Burdin et al., Non-collider searches for stable massive particles, Phys. Rept. 582 (2015) 1 [arXiv:1410.1374] [INSPIRE].

[51] L. Chuzhoy and E.W. Kolb, Reopening the window on charged dark matter, JCAP 07 (2009) 014 [arXiv:0809.0436] [INSPIRE].

[52] P. Langacker and G. Steigman, Requiem for an FCHAMP? Fractionally CHArged, Massive Particle, Phys. Rev. D 84 (2011) 065040 [arXiv:1107.3131] [InSPIRE].

[53] M.L. Perl, E.R. Lee and D. Loomba, Searches for fractionally charged particles, Ann. Rev. Nucl. Part. Sci. 59 (2009) 47 [inSPIRE].

[54] S. Davidson, S. Hannestad and G. Raffelt, Updated bounds on millicharged particles, JHEP 05 (2000) 003 [hep-ph/0001179] [inSPIRE].

[55] O. Antipin, M. Redi, A. Strumia and E. Vigiani, Accidental composite dark matter, arXiv: 1503.08749 [INSPIRE].

[56] L.F. Abbott and P. Sikivie, A cosmological bound on the invisible axion, Phys. Lett. B 120 (1983) 133 [INSPIRE]. 
[57] M. Dine and W. Fischler, The not so harmless axion, Phys. Lett. B 120 (1983) 137 [INSPIRE].

[58] J. Preskill, M.B. Wise and F. Wilczek, Cosmology of the invisible axion, Phys. Lett. B 120 (1983) 127 [INSPIRE].

[59] J. Edsjo and P. Gondolo, Neutralino relic density including coannihilations, Phys. Rev. D 56 (1997) 1879 [hep-ph/9704361] [INSPIRE].

[60] E.W. Kolb and M.S. Turner, The Early universe, Front. Phys. 69 (1990) 1.

[61] J. Hisano, S. Matsumoto, M. Nagai, O. Saito and M. Senami, Non-perturbative effect on thermal relic abundance of dark matter, Phys. Lett. B 646 (2007) 34 [hep-ph/0610249] [INSPIRE].

[62] M. Kawasaki, K. Kohri and T. Moroi, Big-Bang nucleosynthesis and hadronic decay of long-lived massive particles, Phys. Rev. D 71 (2005) 083502 [astro-ph/0408426] [InSPIRE].

[63] C.F. Berger, L. Covi, S. Kraml and F. Palorini, The number density of a charged relic, JCAP 10 (2008) 005 [arXiv: 0807.0211] [InSPIRE].

[64] P. Gondolo and G. Gelmini, Cosmic abundances of stable particles: Improved analysis, Nucl. Phys. B 360 (1991) 145 [inSPIRE].

[65] F. Iocco, G. Mangano, G. Miele, O. Pisanti and P.D. Serpico, Primordial nucleosynthesis: from precision cosmology to fundamental physics, Phys. Rept. 472 (2009) 1 [arXiv: 0809.0631] [INSPIRE].

[66] D. Lindley, Cosmological constraints on the lifetime of massive particles, Astrophys. J. 294 (1985) 1 [INSPIRE].

[67] M.H. Reno and D. Seckel, Primordial nucleosynthesis: the effects of injecting hadrons, Phys. Rev. D 37 (1988) 3441 [INSPIRE].

[68] S. Dimopoulos, R. Esmailzadeh, L.J. Hall and G.D. Starkman, Is the universe closed by baryons? Nucleosynthesis with a late decaying massive particle, Astrophys. J. 330 (1988) 545 [INSPIRE].

[69] R.J. Scherrer and M.S. Turner, Primordial nucleosynthesis with decaying particles. 1. Entropy producing decays. 2. Inert decays, Astrophys. J. 331 (1988) 19 [INSPIRE].

[70] J.R. Ellis, G.B. Gelmini, J.L. Lopez, D.V. Nanopoulos and S. Sarkar, Astrophysical constraints on massive unstable neutral relic particles, Nucl. Phys. B 373 (1992) 399 [INSPIRE].

[71] M. Pospelov, Particle physics catalysis of thermal Big Bang nucleosynthesis, Phys. Rev. Lett. 98 (2007) 231301 [hep-ph/0605215] [INSPIRE].

[72] K. Kohri and F. Takayama, Big bang nucleosynthesis with long lived charged massive particles, Phys. Rev. D 76 (2007) 063507 [hep-ph/0605243] [INSPIRE].

[73] M. Kaplinghat and A. Rajaraman, Big Bang nucleosynthesis with bound states of long-lived charged particles, Phys. Rev. D 74 (2006) 103004 [astro-ph/0606209] [INSPIRE].

[74] C. Bird, K. Koopmans and M. Pospelov, Primordial Lithium abundance in catalyzed Big Bang nucleosynthesis, Phys. Rev. D 78 (2008) 083010 [hep-ph/0703096] [INSPIRE].

[75] T. Jittoh et al., Possible solution to the Li-7 problem by the long lived stau, Phys. Rev. D 76 (2007) 125023 [arXiv:0704.2914] [INSPIRE]. 
[76] K. Jedamzik, The cosmic Li-6 and Li-7 problems and BBN with long-lived charged massive particles, Phys. Rev. D 77 (2008) 063524 [arXiv:0707.2070] [INSPIRE].

[77] T. Jittoh et al., Big-bang nucleosynthesis and the relic abundance of dark matter in a stau-neutralino coannihilation scenario, Phys. Rev. D 78 (2008) 055007 [arXiv:0805.3389] [INSPIRE].

[78] D. Cumberbatch et al., Solving the cosmic lithium problems with primordial late-decaying particles, Phys. Rev. D 76 (2007) 123005 [arXiv:0708.0095] [INSPIRE].

[79] M. Kusakabe, T. Kajino, R.N. Boyd, T. Yoshida and G.J. Mathews, A simultaneous solution to the ${ }^{6} \mathrm{Li}$ and ${ }^{7} \mathrm{Li}$ Big Bang nucleosynthesis problems from a long-lived negatively-charged leptonic particle, Phys. Rev. D 76 (2007) 121302 [arXiv:0711.3854] [INSPIRE].

[80] B.D. Fields, The primordial lithium problem, Ann. Rev. Nucl. Part. Sci. 61 (2011) 47 [arXiv: 1203.3551] [INSPIRE].

[81] G. Steigman, Primordial nucleosynthesis in the precision cosmology era, Ann. Rev. Nucl. Part. Sci. 57 (2007) 463 [arXiv:0712.1100].

[82] R.E. Lopez and M.S. Turner, An accurate calculation of the Big Bang prediction for the abundance of primordial helium, Phys. Rev. D 59 (1999) 103502 [astro-ph/9807279] [INSPIRE].

[83] V.F. Mukhanov, Nucleosynthesis without a computer, Int. J. Theor. Phys. 43 (2004) 669 [astro-ph/0303073].

[84] S. Weinberg, Cosmology, Oxford University Press, Oxford U.K. (2008).

[85] M. Pospelov and J. Pradler, Big Bang nucleosynthesis as a probe of new physics, Ann. Rev. Nucl. Part. Sci. 60 (2010) 539 [arXiv:1011.1054].

[86] C. Englert et al., Precision measurements of higgs couplings: implications for new physics scales, J. Phys. G 41 (2014) 113001 [arXiv:1403.7191] [InSPIRE].

[87] M. Carena, I. Low and C.E.M. Wagner, Implications of a modified Higgs to diphoton decay width, JHEP 08 (2012) 060 [arXiv:1206.1082] [INSPIRE].

[88] I. Picek and B. Radovcic, Enhancement of $h \rightarrow \gamma \gamma$ by seesaw-motivated exotic scalars, Phys. Lett. B 719 (2013) 404 [arXiv: 1210.6449] [INSPIRE].

[89] ATLAS collaboration, Search for doubly-charged Higgs bosons in like-sign dilepton final states at $\sqrt{s}=7 \mathrm{TeV}$ with the ATLAS detector, Eur. Phys. J. C 72 (2012) 2244 [arXiv: 1210.5070] [INSPIRE].

[90] CMS collaboration, A search for a doubly-charged Higgs boson in pp collisions at $\sqrt{s}=7$ TeV, Eur. Phys. J. C 72 (2012) 2189 [arXiv:1207.2666] [InSPIRE].

[91] A. Melfo, M. Nemevšek, F. Nesti, G. Senjanović and Y. Zhang, Type II seesaw at LHC: the roadmap, Phys. Rev. D 85 (2012) 055018 [arXiv:1108.4416] [InSPIRE].

[92] B. Ren, K. Tsumura and X.-G. He, A Higgs quadruplet for type III seesaw and implications for $\mu \rightarrow e \gamma$ and $\mu$-e conversion, Phys. Rev. D 84 (2011) 073004 [arXiv:1107.5879] [INSPIRE].

[93] K.S. Babu, S. Nandi and Z. Tavartkiladze, New mechanism for neutrino mass generation and triply charged Higgs bosons at the LHC, Phys. Rev. D 80 (2009) 071702 [arXiv:0905.2710] [INSPIRE]. 
[94] C. Englert, E. Re and M. Spannowsky, Pinning down Higgs triplets at the LHC, Phys. Rev. D 88 (2013) 035024 [arXiv: 1306.6228] [INSPIRE].

[95] ALEPH collaboration, R. Barate et al., Search for pair production of longlived heavy charged particles in $e^{+} e^{-}$annihilation, Phys. Lett. B 405 (1997) 379 [hep-ex/9706013] [INSPIRE].

[96] DELPHI collaboration, P. Abreu et al., Search for heavy stable and longlived particles in $e^{+} e^{-}$collisions at $\sqrt{s}=189 \mathrm{GeV}$, Phys. Lett. B 478 (2000) 65 [hep-ex/0103038] [INSPIRE].

[97] L3 collaboration, P. Achard et al., Search for heavy neutral and charged leptons in $e^{+} e^{-}$ annihilation at LEP, Phys. Lett. B 517 (2001) 75 [hep-ex/0107015] [InSPIRE].

[98] OPAL collaboration, G. Abbiendi et al., Search for stable and longlived massive charged particles in $e^{+} e^{-}$collisions at $\sqrt{s}=130 \mathrm{GeV}$ to $209 \mathrm{GeV}$, Phys. Lett. B 572 (2003) 8 [hep-ex/0305031] [INSPIRE].

[99] H1 collaboration, A. Aktas et al., Measurement of anti-deuteron photoproduction and a search for heavy stable charged particles at HERA, Eur. Phys. J. C 36 (2004) 413 [hep-ex/0403056] [INSPIRE].

[100] D0 collaboration, V.M. Abazov et al., Search for long-lived charged massive particles with the D0 detector, Phys. Rev. Lett. 102 (2009) 161802 [arXiv:0809.4472] [INSPIRE].

[101] CDF collaboration, T. Aaltonen et al., Search for long-lived massive charged particles in $1.96 \mathrm{TeV} \bar{p} p$ collisions, Phys. Rev. Lett. 103 (2009) 021802 [arXiv:0902.1266] [INSPIRE].

[102] D0 collaboration, V.M. Abazov et al., Search for charged massive long-lived particles at $\sqrt{s}=1.96 \mathrm{TeV}$, Phys. Rev. D 87 (2013) 052011 [arXiv:1211.2466] [INSPIRE].

[103] CMS collaboration, Search for Heavy stable charged particles in pp collisions at $\sqrt{s}=7$ TeV, JHEP 03 (2011) 024 [arXiv:1101.1645] [INSPIRE].

[104] ATLAS collaboration, Search for massive long-lived highly ionising particles with the ATLAS detector at the LHC, Phys. Lett. B 698 (2011) 353 [arXiv:1102.0459] [INSPIRE].

[105] ATLAS collaboration, Search for heavy long-lived charged particles with the ATLAS detector in pp collisions at $\sqrt{s}=7$ TeV, Phys. Lett. B 703 (2011) 428 [arXiv:1106.4495] [INSPIRE].

[106] CMS collaboration, Search for heavy long-lived charged particles in pp collisions at $\sqrt{s}=7$ TeV, Phys. Lett. B 713 (2012) 408 [arXiv:1205. 0272] [InSPIRE].

[107] ATLAS collaboration, Search for long-lived, multi-charged particles in pp collisions at $\sqrt{s}=7 \mathrm{TeV}$ using the ATLAS detector, Phys. Lett. B 722 (2013) 305 [arXiv:1301.5272] [INSPIRE].

[108] CMS collaboration, Searches for long-lived charged particles in pp collisions at $\sqrt{s}=7$ and $8 \mathrm{TeV}$, JHEP 07 (2013) 122 [arXiv: 1305.0491] [INSPIRE].

[109] CMS collaboration, Reinterpreting the results of the search for long-lived charged particles in the pMSSM and other BSM scenarios, CMS-PAS-EXO-13-006 (2013).

[110] A.D. Martin, W.J. Stirling, R.S. Thorne and G. Watt, Parton distributions for the LHC, Eur. Phys. J. C 63 (2009) 189 [arXiv:0901.0002] [InSPIRE].

[111] MoEDAL collaboration, J. Pinfold et al., Technical design report of the MoEDAL experiment, CERN-LHCC-2009-006 (2009). 
[112] MoEDAL collaboration, B. Acharya et al., The physics programme of the MoEDAL experiment at the LHC, Int. J. Mod. Phys. A 29 (2014) 1430050 [arXiv:1405.7662] [INSPIRE].

[113] CMS collaboration, Search for dark matter, extra dimensions and unparticles in monojet events in proton-proton collisions at $\sqrt{s}=8$ TeV, Eur. Phys. J. C 75 (2015) 235 [arXiv: 1408.3583] [INSPIRE].

[114] N. Zhou, D. Berge and D. Whiteson, Mono-everything: combined limits on dark matter production at colliders from multiple final states, Phys. Rev. D 87 (2013) 095013 [arXiv:1302.3619] [INSPIRE].

[115] ALEPH, DELPHI, L3, OPAL, LEP Electroweak Working Group collaboration, J. Alcaraz et al., A combination of preliminary electroweak measurements and constraints on the standard model, hep-ex/0612034 [INSPIRE].

[116] Particle Data Group collaboration, K. Olive et al., Review of particle physics, Chin. Phys. C 38 (2014) 090001.

[117] ATLAS collaboration, Search for charginos nearly mass degenerate with the lightest neutralino based on a disappearing-track signature in pp collisions at $\sqrt{s}=8 \mathrm{TeV}$ with the ATLAS detector, Phys. Rev. D 88 (2013) 112006 [arXiv:1310.3675] [INSPIRE].

[118] ALEPH collaboration, A. Heister et al., Search for charginos nearly mass degenerate with the lightest neutralino in $e^{+} e^{-}$collisions at center-of-mass energies up to $209 \mathrm{GeV}$, Phys. Lett. B 533 (2002) 223 [hep-ex/0203020] [INSPIRE].

[119] DELPHI collaboration, P. Abreu et al., Update of the search for charginos nearly mass-degenerate with the lightest neutralino, Phys. Lett. B 485 (2000) 95 [hep-ex/0103035] [INSPIRE].

[120] L3 collaboration, M. Acciarri et al., Search for charginos with a small mass difference with the lightest supersymmetric particle at $\sqrt{S}=189 \mathrm{GeV}$, Phys. Lett. B 482 (2000) 31 [hep-ex/0002043] [INSPIRE].

[121] OPAL collaboration, G. Abbiendi et al., Search for nearly mass degenerate charginos and neutralinos at LEP, Eur. Phys. J. C 29 (2003) 479 [hep-ex/0210043] [INSPIRE].

[122] E. Conte, B. Fuks and G. Serret, MadAnalysis 5, a user-friendly framework for collider phenomenology, Comput. Phys. Commun. 184 (2013) 222 [arXiv:1206.1599] [INSPIRE].

[123] OPAL collaboration, G. Abbiendi et al., Search for chargino and neutralino production at $\sqrt{s}=192 \mathrm{GeV}$ to $209 \mathrm{GeV}$ at LEP, Eur. Phys. J. C 35 (2004) 1 [hep-ex/0401026] [INSPIRE].

[124] M. Fairbairn et al., Stable massive particles at colliders, Phys. Rept. 438 (2007) 1 [hep-ph/0611040] [INSPIRE].

[125] T. Sjöstrand, S. Mrenna and P.Z. Skands, PYTHIA 6.4 physics and manual, JHEP 05 (2006) 026 [hep-ph/0603175] [INSPIRE].

[126] S. Moretti, K. Odagiri, P. Richardson, M.H. Seymour and B.R. Webber, Implementation of supersymmetric processes in the HERWIG event generator, JHEP 04 (2002) 028 [hep-ph/0204123] [INSPIRE].

[127] J. Bellm et al., HERWIG++ 2.7 release note, arXiv:1310.6877 [INSPIRE]. 
[128] A. De Rujula, H. Georgi and S.L. Glashow, Hadron masses in a gauge theory, Phys. Rev. D 12 (1975) 147 [INSPIRE].

[129] A.C. Kraan, Interactions of heavy stable hadronizing particles, Eur. Phys. J. C 37 (2004) 91 [hep-ex/0404001] [INSPIRE].

[130] M. Drees and X. Tata, Signals for heavy exotics at hadron colliders and supercolliders, Phys. Lett. B 252 (1990) 695 [INSPIRE].

[131] A. Mafi and S. Raby, An analysis of a heavy gluino LSP at CDF: the heavy gluino window, Phys. Rev. D 62 (2000) 035003 [hep-ph/9912436] [INSPIRE].

[132] H. Baer, K.-m. Cheung and J.F. Gunion, A heavy gluino as the lightest supersymmetric particle, Phys. Rev. D 59 (1999) 075002 [hep-ph/9806361] [INSPIRE].

[133] R. Mackeprang and A. Rizzi, Interactions of coloured heavy stable particles in matter, Eur. Phys. J. C 50 (2007) 353 [hep-ph/0612161] [INSPIRE].

[134] R. Mackeprang and D. Milstead, An updated description of heavy-hadron interactions in GEANT-4, Eur. Phys. J. C 66 (2010) 493 [arXiv:0908.1868] [INSPIRE].

[135] ATLAS collaboration, Searches for heavy long-lived charged particles with the ATLAS detector in proton-proton collisions at $\sqrt{s}=8 \mathrm{TeV}$, JHEP 01 (2015) 068 [arXiv: 1411.6795] [INSPIRE].

[136] CMS collaboration, Search for decays of stopped long-lived particles produced in proton-proton collisions at $\sqrt{s}=8 \mathrm{TeV}$, Eur. Phys. J. C 75 (2015) 151 [arXiv: 1501.05603] [INSPIRE].

[137] ATLAS collaboration, Search for long-lived stopped R-hadrons decaying out-of-time with pp collisions using the ATLAS detector, Phys. Rev. D 88 (2013) 112003 [arXiv:1310.6584] [INSPIRE].

[138] A. Arvanitaki, S. Dimopoulos, A. Pierce, S. Rajendran and J.G. Wacker, Stopping gluinos, Phys. Rev. D 76 (2007) 055007 [hep-ph/0506242] [INSPIRE].

[139] R.N. Mohapatra and G. Senjanović, Neutrino mass and spontaneous parity violation, Phys. Rev. Lett. 44 (1980) 912 [INSPIRE].

[140] G. D'Ambrosio, G.F. Giudice, G. Isidori and A. Strumia, Minimal flavor violation: an effective field theory approach, Nucl. Phys. B 645 (2002) 155 [hep-ph/0207036] [InSPIRE].

[141] K. Abe, T. Abe, H. Aihara, Y. Fukuda, Y. Hayato et al., Letter of intent: the Hyper-Kamiokande experiment - Detector design and physics potential, arXiv:1109.3262 [INSPIRE].

[142] A. Garfagnini, Neutrinoless double beta decay experiments, arXiv:1408.2455 [INSPIRE].

[143] M.E. Machacek and M.T. Vaughn, Two loop renormalization group equations in a general quantum field theory. 1. Wave function renormalization, Nucl. Phys. B 222 (1983) 83 [INSPIRE].

[144] L.N. Mihaila, J. Salomon and M. Steinhauser, Renormalization constants and $\beta$-functions for the gauge couplings of the standard model to three-loop order, Phys. Rev. D 86 (2012) 096008 [arXiv: 1208.3357] [INSPIRE]. 